\title{
TESS cycle 1 observations of roAp stars with 2-min cadence data
}

\author{
D. L. Holdsworth ${ }^{\circledR},{ }^{1 \star}$ M. S. Cunha, ${ }^{2}$ D. W. Kurtz ${ }^{\circledR},{ }^{1,3}$ V. Antoci, ${ }^{4,5}$ D. R. Hey, ${ }^{5,6}$ D. M. Bowman ${ }^{\circledR}, 7$ \\ O. Kobzar, ${ }^{8}$ D. L. Buzasi, ${ }^{9}$ O. Kochukhov ${ }^{\circledR},{ }^{10}$ E. Niemczura, ${ }^{11}$ D. Ozuyar, ${ }^{12}$ F. Shi ${ }^{\circledR},{ }^{13,14}$ R. Szabó, ${ }^{15,16,17}$ \\ A. Samadi-Ghadim, ${ }^{18,19}$ Zs. Bognár, ${ }^{15,16,17}$ L. Fox-Machado, ${ }^{20}$ V. Khalack, ${ }^{8}$ M. Lares-Martiz ${ }^{\circledR},{ }^{21}$ \\ C. C. Lovekin, ${ }^{22}$ P. Mikołajczyk, ${ }^{11}$ D. Mkrtichian, ${ }^{23}$ J. Pascual-Granado ${ }^{\circledR},{ }^{21}$ E. Paunzen ${ }^{\circledR},{ }^{24}$ \\ T. Richey-Yowell, ${ }^{25}$ Á. Sódor, ${ }^{15,16}$ J. Sikora, ${ }^{26}$ T. Z. Yang, ${ }^{27}$ E. Brunsden, ${ }^{28,29}$ A. David-Uraz ${ }^{\circledR},{ }^{30,31}$
} A. Derekas, ${ }^{32,33}$ A. García Hernández, ${ }^{34}$ J. A. Guzik, ${ }^{35}$ N. Hatamkhani, ${ }^{36}$ R. Handberg ${ }^{\circledR}, 5$ T. S. Lambert ${ }^{\circledR},{ }^{37}$ P. Lampens, ${ }^{38}$ S. J. Murphy ${ }^{\circledR},{ }^{5,6}$ R. Monier, ${ }^{39}$ K. R. Pollard, ${ }^{29}$ P. Quitral-Manosalva, ${ }^{40}$ A. Ramón-Ballesta, ${ }^{21}$ B. Smalley ${ }^{\circledR},{ }^{41}$ I. Stateva ${ }^{42}$ and R. Vanderspek ${ }^{43}$

Affiliations are listed at the end of the paper

Accepted 2021 May 27. Received 2021 May 27; in original form 2021 May 12

\begin{abstract}
We present the results of a systematic search for new rapidly oscillating Ap (roAp) stars using the 2-min cadence data collected by the Transiting Exoplanet Survey Satellite (TESS) during its Cycle 1 observations. We identify 12 new roAp stars. Amongst these stars we discover the roAp star with the longest pulsation period, another with the shortest rotation period, and six with multiperiodic variability. In addition to these new roAp stars, we present an analysis of 44 known roAp stars observed by TESS during Cycle 1, providing the first high-precision and homogeneous sample of a significant fraction of the known roAp stars. The TESS observations have shown that almost 60 per cent (33) of our sample of stars are multiperiodic, providing excellent cases to test models of roAp pulsations, and from which the most rewarding asteroseismic results can be gleaned. We report four cases of the occurrence of rotationally split frequency multiplets that imply different mode geometries for the same degree modes in the same star. This provides a conundrum in applying the oblique pulsator model to the roAp stars. Finally, we report the discovery of non-linear mode interactions in $\alpha$ Cir (TIC 402546736, HD 128898) around the harmonic of the principal mode - this is only the second case of such a phenomenon.
\end{abstract}

Key words: asteroseismology - techniques: photometric - stars: chemically peculiar - stars: magnetic field - stars: oscillations stars: variables.

\section{INTRODUCTION}

The rapidly oscillating Ap (roAp) stars are a rare subset of the chemically peculiar, magnetic, Ap stars. They are found at the base of the classical instability strip where it intersects the main sequence, and range in age from the zero-age main sequence to beyond the terminal-age main sequence. Since their discovery (Kurtz 1978 , 1982) there have been several attempts to enlarge, and study, the sample of roAp stars. These have included targeted and survey ground-based photometry (e.g. Martinez, Kurtz \& Kauffmann 1991; Nelson \& Kreidl 1993; Martinez \& Kurtz 1994b; Paunzen et al. 2012; Holdsworth et al. 2014a; Joshi et al. 2016; Paunzen et al. 2018), time-resolved high resolution spectroscopic observations (e.g. Elkin, Kurtz \& Mathys 2008; Freyhammer et al. 2008a; Kochukhov et al. 2013) and most recently space photometry (e.g. Bruntt et al. 2009; Balona et al. 2011; Gruberbauer et al. 2011; Kurtz et al. 2011; Weiss et al. 2016; Balona, Holdsworth \& Cunha 2019; Cunha et al. 2019;

^E-mail: DLHoldsworth@uclan.ac.uk
Hey et al. 2019; Holdsworth 2021). To date, including the results presented here, there are 88 roAp stars known in the literature.

The overarching class of Ap stars hosts strong, stable, global magnetic fields that can have field strengths of up to $34 \mathrm{kG}$ (Babcock 1960; Mathys 2017), with the magnetic axis misaligned with respect to the rotation axis. The magnetic field is thought to be the main factor in braking the rotation velocity of the star (Stępień 2000), such that the Ap stars show rotation periods of between a few days and perhaps centuries (North 1984; Sikora et al. 2019c; Mathys, Kurtz \& Holdsworth 2020b). The presence of the magnetic field also serves to suppress near-surface convection, allowing for the stratification of elements in the stellar atmosphere (Michaud, Megessier \& Charland 1981; LeBlanc et al. 2009; Alecian \& Stift 2010) through the effects of radiative levitation and gravitational settling. In particular, the radiative levitation leads to surface inhomogeneities of elements such as $\mathrm{Ce}, \mathrm{Pr}, \mathrm{Nd}, \mathrm{Sm}, \mathrm{Eu}$, and $\mathrm{Tb}$, which can be overabundant by over a million times the solar value (e.g. Lüftinger et al. 2010).

These chemical spots, which are either bright or dark depending on the wavelength of observation, cause non-sinusoidal modulation of the light curve over the stellar rotation period. This allows for precise rotation periods to be measured, and is well represented 
by the oblique rotator model (Stibbs 1950). Such a modulation is also seen in the magnetic field strength (e.g. Mathys 2017; Hubrig et al. 2018), and spectral line strength. These rotationally modulated stars are commonly known as $\alpha^{2}$ CVn stars (Samus' et al. 2017). Kochukhov (2011) concluded that the distribution of the chemical spots of the Ap stars is diverse, rarely showing a connection with the magnetic field structure.

The pulsations in the roAp stars are high overtone ( $n \gtrsim 15)$, low degree $(\ell \lesssim 3)$ pressure $(\mathrm{p})$ modes with frequencies in the range $0.7-3.6 \mathrm{mHz}\left(60-310 \mathrm{~d}^{-1} ; P=4.7-23.6 \mathrm{~min}\right)$. The pulsation axis in these stars is misaligned to both the rotation and magnetic axes, leading to the development of the oblique pulsator model (Kurtz 1982; Shibahashi \& Saio 1985; Bigot \& Dziembowski 2002; Bigot $\&$ Kurtz 2011). Such a configuration means that the pulsation mode is viewed from varying aspects over the rotation cycle of the star, leading to an apparent amplitude modulation of the mode. In a Fourier spectrum of a light curve, a multiplet is seen with $2 \ell+1$ components for a non-distorted mode, with the components split from the central mode frequency by exactly the stellar rotation frequency.

The driving mechanism for the pulsations in roAp stars is still an area of active research. In many cases, it is currently thought that the pulsations are driven by the opacity $(\kappa)$ mechanism in the hydrogen ionization layers in regions of the star where the magnetic field suppresses convection (Balmforth et al. 2001). However, this theory cannot reproduce observations of the highest frequency modes in some stars. In these cases Cunha et al. (2013) proposed that a mechanism linked to turbulent pressure plays a role in the excitation of these highest frequency modes.

A further conundrum linked to the excitation mechanism of the pulsations in roAp stars comes with the inspection of the theoretical instability strip of these stars. Cunha (2002), based on the models of Balmforth et al. (2001), calculated the extent of the theoretical instability strip for roAp stars and found a much hotter blue edge than the observations mapped, and that some known roAp stars were cooler than the red edge. It is still unclear why the theoretical models do not match the observational evidence.

Of particular scientific interest are the roAp stars which show multiple pulsation modes as these stars provide the ability to constrain structure models of Ap stars. In the absence of a magnetic field, the pulsation modes of an roAp star would form a series of alternating degree modes that are equally spaced in frequency, as is expected in the asymptotic regime for high radial-order acoustic oscillations (Shibahashi 1979; Tassoul 1980). The acoustic waves in the outer layers of the roAp stars, in the presence of the strong magnetic field, become magnetoacoustic in nature which consequently changes the mode frequencies (Cunha \& Gough 2000; Saio \& Gautschy 2004; Cunha 2006). In most cases, the frequency separation between consecutive modes is not significantly affected, still enabling the use of asteroseismic techniques to help constrain the stellar properties as would be done for non-magnetic stars (Cunha, Fernandes \& Monteiro 2003; Deal et al. 2021). Nevertheless, at some particular frequencies the coupling between the magnetic field and pulsations is optimal and the pulsation spectrum can be significantly modified. It is the multiperiodic stars, which in some cases show significant shifts from the expected non-magnetic frequency pattern, that provide the most scientific return (e.g. Cunha 2001; Gruberbauer et al. 2008).

The Transiting Exoplanet Survey Satellite (TESS; Ricker et al. 2015 ) is surveying almost the entire sky, in 27-d long strips known as sectors, in the search for transiting exoplanets. As such, it is collecting high precision photometric data on millions of stars at 2min and 30-min cadences (with a 20-s cadence added in the extended mission and reducing the full-frame image cadence to $10 \mathrm{~min}$ ). The Cycle 1 observations, conducted in the first year of the primary mission, covered the southern ecliptic hemisphere, with Cycle 2 observing the northern ecliptic hemisphere. Such an observational data set provides the opportunity to perform an homogeneous search for high-frequency pulsations in a significant selection of stars.

This work utilizes the 2-min cadence TESS Cycle 1 observations (sectors 1 through 13) to search for high-frequency pulsations in main sequence stars hotter than $6000 \mathrm{~K}$. We present the observational results here, and will, in a future work, use these results to perform an ensemble study of the properties of the roAp stars. The paper is laid out as follows: in Section 2, we introduce our data sample; in Section 3, we provide details of spectroscopic follow up observations; in Section 4, we present the results of our search. In Section 5, we present candidate roAp stars where we have insufficient data to confirm their true nature, and in Section 6, we draw our conclusions.

\section{DATA SAMPLE AND SEARCH STRATEGY}

To construct our catalogue of target stars, we took the 2-min target list (constructed from proposals from the exoplanet and asteroseismic communities) for each sector of observations from TESS ${ }^{1}$ which consisted of 20000 stars per sector. These lists were then crossmatched with version 8 of the TESS Input Catalog (TIC; Stassun et al. 2019) with a 3 arcsec radius. This gave us access to the secondary information in the TIC to refine our targets. Using only the $T_{\text {eff }}$ parameter, we selected stars with temperatures of $6000 \mathrm{~K}$ or hotter. This temperature cut is to ensure we do not exclude around the red edge of the roAp star instability strip, and to account for any inaccuracies in the TIC temperature values. This amounted to about 7500 stars per sector, with 101847 light curves in total, corresponding to 50703 unique stars (with 15363 stars observed in more than one sector). The light curves for each star in all available sectors were downloaded from the Mikulski Archive for Space Telescopes (MAST) server. These data have been processed with the Science Processing Operations Center (SPOC) pipeline (Jenkins et al. 2016). In the following, we used the Pre-search Data Conditioning Simple Aperture Photometry (PDC_SAP) data unless otherwise stated.

Initially, three independent analysis techniques were implemented for the search of pulsational variability. For one team, each individual light curve was automatically pre-whitened to a frequency of $0.23 \mathrm{mHz}\left(20 \mathrm{~d}^{-1}\right)$ to an amplitude limit of the approximate noise level between 2.3 and $3.3 \mathrm{mHz}\left(200-300 \mathrm{~d}^{-1}\right)$ to remove instrumental artefacts and any low-frequency signal whose window function affects the noise at high frequency. If multiple sectors for a target were available, the pre-whitened sectors were then combined. An amplitude spectrum of the light curve was calculated to the Nyquist frequency of $4.2 \mathrm{mHz}\left(360 \mathrm{~d}^{-1}\right)$, which also included a calculation of the false alarm probability (FAP).

From these amplitude spectra, we selected stars which showed peaks with frequencies $>0.52 \mathrm{mHz}\left(>45 \mathrm{~d}^{-1}\right)$ and a corresponding FAP of $<0.1$. The amplitude spectra of these stars were then plotted for visual inspection. This totalled 6713 stars, of which 2125 stars have multisector observations.

A second team used a complementary method which calculated the skewness of the amplitude spectrum (Murphy et al. 2019) of the MAST PDC_SAP data above $0.46 \mathrm{mHz}\left(40 \mathrm{~d}^{-1}\right)$. Where multiple sectors of data were available, they were combined to a single light curve. If the skewness was greater than 5 , then all peaks in the

\footnotetext{
${ }^{1}$ https://tess.mit.edu/observations/target-lists/
} 
amplitude spectrum with FAP values below 0.05 were extracted and the star flagged as variable for later human inspection. This method produced a total of 189 variable star candidates.

The third team used the TESS-AP automated analysis procedure created by Khalack et al. (2019). The light curves of stars with TIC effective temperatures in the range $7000-10000 \mathrm{~K}$ were analysed using the PERIOD04 package (Lenz \& Breger 2005) in an automated way. A star was flagged as a candidate if a signal was found in the $1.16-4.17 \mathrm{mHz}\left(100-360 \mathrm{~d}^{-1}\right)$ range with an amplitude three times that of the local noise. These candidates were then checked by eye before being included in a final list. The final count of variable star candidates was 78 .

These three lists were then combined to produce a master list where each star and light curve was subjected to human inspection. An initial pass was made to remove false positive detections. A detection was determined to be false positive if the $\mathrm{S} / \mathrm{N}$ of the highfrequency signal was below 4.0; if there was obvious contamination from a low-frequency harmonic series; or if the amplitude spectrum displayed obvious characteristics of either $\delta$ Sct stars (with many modes in the $\approx 0.2-1.0 \mathrm{mHz}$ range), sdBV stars (stars with pulsations in low and high-frequency ranges) or pulsating white dwarfs (modes with amplitudes significantly greater than those known in roAp stars). The final sample consisted of 163 stars.

This final sample was distributed amongst the members of the TESS Asteroseismic Science Consortium (TASC) Working Group 4 (WG4) for detailed analysis to confirm the presence of a positive roAp detection, and to extract rotation periods from the light curves. This process identified 12 new roAp stars previously unreported in the literature and 10 roAp stars discovered through TESS observations and reported by either Cunha et al. (2019) or Balona et al. (2019). There are also positive detections of pulsations in 31 roAp stars known prior to the launch of TESS, with 3 roAp stars where TESS did not detect pulsational variability as their low amplitude modes are below the TESS detection limit. Finally, we present 5 roAp candidate stars where there is inconclusive evidence as to whether the star is truly an roAp pulsator.

\section{SPECTROSCOPIC OBSERVATIONS}

For a subset of our sample, we have made new spectroscopic observations to confirm or detect the chemically peculiar nature of the star. Most of these observations were obtained with the SpUpNIC long-slit spectrograph (Crause et al. 2016) mounted on the 1.9-m telescope of the South African Astronomical Observatory (SAAO). The observations were made in the blue part of the spectrum $(\sim 3900-$ $5100 \AA$ ) with grating 4 which achieves a resolution of about $0.6 \AA$ per pixel, with some stars also having a red spectrum $(\sim 6100$ $7150 \AA$ ) obtained with grating 6 with a resolution of $1.4 \AA$ per pixel. The data were reduced in the standard way, ${ }^{2}$ with wavelength calibrations provided by arc spectra obtained directly after the science observation.

Where possible, we also observed some stars with the Southern African Large Telescope (SALT; Buckley, Swart \& Meiring 2006), utilizing the High Resolution Spectrograph (HRS; Bramall et al. 2010; Crause et al. 2014). HRS is a dual-beam spectrograph with wavelength coverage of 3700-5500 $\AA$ and 5500-8900 $\AA$. The observations were obtained in either the medium $(R \sim 43000)$ or high $(R \sim 63000)$ resolution modes, and automatically reduced using

\footnotetext{
${ }^{2} \mathrm{http}: / /$ www.starlink.ac.uk/docs/sc7.htx/sc7.html
}

the SALT custom pipeline, which is based on the European Southern Observatory's (ESO) MIDAS pipeline (Kniazev, Gvaramadze \& Berdnikov 2016, 2017).

Finally, we also used the High Efficiency and Resolution Canterbury University Large Échelle Spectrograph (HERCULES) at the 1.0-m McLellan Telescope of the University of Canterbury Mt John Observatory (Hearnshaw et al. 2003). HERCULES has a wavelength coverage of 3800-8800 $\AA$ with observations made at $R=41000$. The observations were automatically reduced using the HERCULES custom pipeline, $\{\backslash \mathrm{sc}$ megara $\}$. We provide a log of all spectroscopic observations in Table A1.

Spectral classification with these observations was made by comparison with MK standard stars, following the procedure of Gray \& Corbally (2009). The observations were normalized using an automated spline fitting procedure in the iSPEC spectral analysis software (Blanco-Cuaresma et al. 2014; Blanco-Cuaresma 2019), with the MK standards plotted for comparison. With the classification as a starting point, we computed synthetic spectra using iSPEC to estimate effective temperatures. The synthesis used the SPECTRUM code (Gray \& Corbally 1994) with a MARCS Gaia-ESO survey model atmosphere (Gustafsson et al. 2008; Gilmore et al. 2012) and a VALD line list (Ryabchikova et al. 2015). Spectra were synthesized with a fixed $\log g=4.0\left(\mathrm{~cm} \mathrm{~s}^{-2}\right)$, solar metallicity and zero microturbulence and macroturbulence. Although these parameters are not refined for each star, they are adequate given the low resolution of most of the data. Spectra were synthesized in temperature steps of $100 \mathrm{~K}$, with the result taken as the best fit to the wings of the observed Balmer lines.

\section{RESULTS}

Here we present the results of our search and analysis of new and known roAp stars in TESS Cycle 1 data. We provide an overview of the targets to be discussed in Table 1 and divide this section, and the table, into subsections addressing new discoveries reported in this work, previous discoveries reliant on the TESS data, and TESS observations of known roAp stars. Finally, we provide a list of candidate roAp stars where the currently available data do not allow us to confirm the nature of these stars.

Throughout this section, to keep homogeneity of the results, we present the numerical results of a single team, hereafter named the reference team. These results have been corroborated by the WG4 contribution to the analysis of the target stars. Where discrepancies were identified between the reference team and the WG4 members, secondary checks were conducted to ensure accurate results are presented.

To avoid repetition, we outline here the process by which each star was analysed in detail, unless a different method is detailed in the appropriate star's subsection. Where available, we combined multisector observations to produce a single light curve for analysis. Using the original PDC_SAP data, we calculated a Discrete Fourier Transform, following Kurtz (1985) in the low-frequency range (to $\left.0.11 \mathrm{mHz} ; 10 \mathrm{~d}^{-1}\right)$ in the search for rotational variability. Where present, we fitted a harmonic series by non-linear least squares to determine the rotation frequency. Where we found a harmonic to have a stronger signal, we derived the rotation frequency from that signal as it can be more precisely determined. Where no rotational variability was found, we also checked the SAP light curve for signs of rotation as the SPOC pipeline can mistakenly remove lowfrequency astrophysical signal.

Subsequently, with the astrophysical information extracted, we removed the rotation signal and any instrumental artefacts through 
Table 1. Details of the stars analysed in this paper. The columns provide the TIC identifier and star name, the TIC v8.1 TESS magnitude, the spectral type as referenced in the stars discussion section, where ${ }^{* *}$ ' denotes a classification derived in this work, the effective temperature, as provided in the TIC, the sectors in which TESS observed the target in Cycle 1. The final three columns provide the stellar rotation period derived in this work, with a ${ }^{\dagger}$ denoting the first detection of this period, the pulsation frequency(ies) found in this work (note that sidelobes that arise from oblique pulsation are not listed), and the pulsation amplitudes

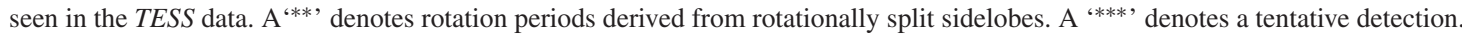

\begin{tabular}{|c|c|c|c|c|c|c|c|c|}
\hline TIC & $\begin{array}{c}\mathrm{HD} / \mathrm{TYC} \\
\text { name }\end{array}$ & $\begin{array}{c}\text { TESS } \\
\text { mag }\end{array}$ & $\begin{array}{l}\text { Spectral } \\
\text { type }\end{array}$ & $\begin{array}{l}T_{\text {eff }}^{\mathrm{TIC}} \\
(\mathrm{K})\end{array}$ & Sectors & $\begin{array}{l}P_{\text {rot }} \\
\text { (d) }\end{array}$ & $\begin{array}{l}\text { Pulsation frequency } \\
\qquad(\mathrm{mHz})\end{array}$ & $\begin{array}{l}\text { Pulsation amplitude } \\
\text { (mmag) }\end{array}$ \\
\hline
\end{tabular}

New TESS roAp stars found in this work

$96315731-51203-10.18$

Ap SrEuCr $\quad 7100 \quad 6,7 \quad 6.6713 \pm 0.0007$

$\begin{array}{lll}119327278 & 45698 & 8.08\end{array}$

$\begin{array}{lcc}170586794 & 107619 & 8.47 \\ 176516923 & 38823 & 7.12\end{array}$

$\mathrm{A} 2 \mathrm{p} \mathrm{SrEu} \quad 8540$

$\begin{array}{rrr}6,7 & 6.6713 & \pm 0.0007 \\ 6,7 & 1.08457 & \pm 0.00003\end{array}$

$\begin{array}{llll}\text { F5 p EuCr* } & 6590 & 10 & 10.31 \pm 0.04^{\dagger}\end{array}$

A5p SrEuCr 7660

10
6

$8.782 \pm 0.003$

$\begin{array}{ccccccc}178575480 & 55852 & 8.83 & \text { Ap SrEuCr } & 7720 & 7 & 4.7788 \pm 0.0005 \\ 294266638 & 6021-415-1 & 9.74 & \text { A7p SrEu* } & 7230 & 8 & \text { No signature } \\ & & & & & \\ 294769049 & 161423 & 9.17 & \mathrm{Ap} \mathrm{SrEu}(\mathrm{Cr}) & 7760 & 12,13 & 10.4641 \pm 0.0007^{\dagger} \\ 310817678 & 88507 & 9.60 & \mathrm{Ap} \mathrm{SrEu}(\mathrm{Cr}) & 8230 & 9,10 & 2.75003 \pm 0.00008^{\dagger}\end{array}$

$\begin{array}{ccc}356088697 & 76460 & 9.53 \\ 380651050 & 176384 & 8.19 \\ 387115314 & 9462-347-1 & 9.56\end{array}$

$466260580 \quad 9087-1516-1 \quad 11.64$
roAp stars previously discovered by TESS

$\begin{array}{lll}\text { roAp stars previously discovered by TESS } \\ 12968953 & 217704 & 10.09\end{array}$

$\mathrm{Ap} \mathrm{EuCr*}$

6830

13

No signature

A7p SrEuCr*

7880

2

No signature

$\begin{array}{lcc}17676722 & 63773 & 8.61 \\ 41259805 & 43226 & 8.84 \\ & & \\ 49818005 & 19687 & 9.36 \\ 152808505 & 216641 & 7.88\end{array}$

A3p SrEuCr*

8320

$\mathrm{A} 6 \mathrm{p} \mathrm{SrEu}(\mathrm{Cr})^{*}$

8360

7

1-8, 10-13

$1.5995 \pm 0.0003$

$\mathrm{F} 2 \mathrm{pSrEu}(\mathrm{Cr})^{*} \quad 7300$

F3p EuCr*

6430

4
1

A8p SrEuCr*

7540

6,7

$6.8580 \pm 0.0003$

$\begin{array}{lllllll}259587315 & 30849 & 8.69 & \text { Ap SrCrEu } & 7720 & 4,5 & 15.776 \pm 0.005 \\ 349945078 & 57040 & 8.92 & \text { A2p EuCr } & 7200 & 6-9 & 13.4256 \pm 0.0008 \\ 350146296 & 63087 & 9.17 & \text { F0p EuCr* } & 7680 & 1-13 & 2.66387 \pm 0.00002\end{array}$

431380369

20880

7.82

Ap $\operatorname{Sr}(\mathrm{EuCr})$

8230

$2,6,13$

$5.19716 \pm 0.00005$

Known roAp stars prior to TESS launch

$6118924 \quad 116114 \quad 6.77$

$\begin{array}{lll}33601621 & 42659 & 6.60\end{array}$
$1.91271 \pm 0.00001$
$0.20583 \pm 0.00001$
$2.43755 \pm 0.00001$
$1.76151 \pm 0.00003$
$1.76019 \pm 0.00005$
$1.85820 \pm 0.00004$
$1.91302 \pm 0.00005$
$2.23870 \pm 0.00004$
$2.39524 \pm 0.00004$
$1.56421 \pm 0.00002$
$1.62882 \pm 0.00002$
$2.26044 \pm 0.00001$
$1.170979 \pm 0.000019$
$1.205272 \pm 0.000003$
$1.229890 \pm 0.000030$
$0.646584 \pm 0.000008$
$1.85817 \pm 0.00002$
$1.87771 \pm 0.00002$
$1.319020 \pm 0.000039$
$1.328382 \pm 0.000020$
$1.356225 \pm 0.000006$
$1.384083 \pm 0.000007$
$1.340060 \pm 0.00002$

$0.125+0.011$

$0.036 \pm 0.004$

$0.041 \pm 0.004$

$0.045 \pm 0.006$

$0.023 \pm 0.004$

$0.006 \pm 0.004$

$0.023 \pm 0.004$

$0.010 \pm 0.007$

$0.052 \pm 0.008$

$0.119 \pm 0.012$

$0.149 \pm 0.012$

$0.054 \pm 0.006$

$0.049 \pm 0.032$

$0.337 \pm 0.032$

$0.133 \pm 0.032$

$0.053 \pm 0.006$

$0.059 \pm 0.004$

$0.045 \pm 0.004$

$0.051 \pm 0.009$

$0.101 \pm 0.009$

$0.375 \pm 0.009$

$0.274 \pm 0.009$

$0.453 \pm 0.035$

$1.27047 \pm 0.00005$

$1.25875 \pm 0.00004$

$1.26961 \pm 0.00003$

$1.33782 \pm 0.00003$

$1.941545 \pm 0.000002$

$2.311039 \pm 0.000001$

$2.326368 \pm 0.000003$

$1.641910 \pm 0.000008$

$1.36260 \pm 0.00005$

$1.38593 \pm 0.00002$

$1.39119 \pm 0.00002$

$0.063251 \pm 0.000002$

$1.274574 \pm 0.000021$

$1.302738 \pm 0.000014$

$1.317816 \pm 0.000008$

$0.904064 \pm 0.000009$

$0.905469 \pm 0.000013$

$0.931050 \pm 0.000003$

$0.950735 \pm 0.000022$

$2.126381 \pm 0.000002$

$2.186645 \pm 0.000005$

$3.4031468 \pm 0.0000078$

$3.4424674 \pm 0.0000021$

$3.4839854 \pm 0.0000015$

$3.5232955 \pm 0.0000005$

$3.5626041 \pm 0.0000031$

$0.805252 \pm 0.000002$

$0.815837 \pm 0.000002$

$0.860412 \pm 0.000001$

$0.872662 \pm 0.000003$

$0.071 \pm 0.013$

$0.083 \pm 0.013$

$0.064 \pm 0.013$

$0.099 \pm 0.013$

$0.671 \pm 0.006$

$0.025 \pm 0.002$

$0.013 \pm 0.002$

$0.267 \pm 0.009$

$0.019 \pm 0.004$

$0.054 \pm 0.004$

$0.041 \pm 0.004$

$0.366 \pm 0.006$

$0.039 \pm 0.006$

$<0.020$

$0.107 \pm 0.006$

$0.071 \pm 0.005$

$0.050 \pm 0.005$

$0.154 \pm 0.005$

$0.029 \pm 0.005$

$0.140 \pm 0.004$

$0.063 \pm 0.004$

$0.005 \pm 0.002$

$0.020 \pm 0.002$

$0.026 \pm 0.002$

$0.081 \pm 0.002$

$0.013 \pm 0.002$

$0.022 \pm 0.003$

$0.016 \pm 0.003$

$0.032 \pm 0.003$

$0.013 \pm 0.003$

$0.76923 \pm 0.00004$

$1.74757 \pm 0.00003$
$0.020 \pm 0.003$

$0.039 \pm 0.005$ 
Table 1 - continued

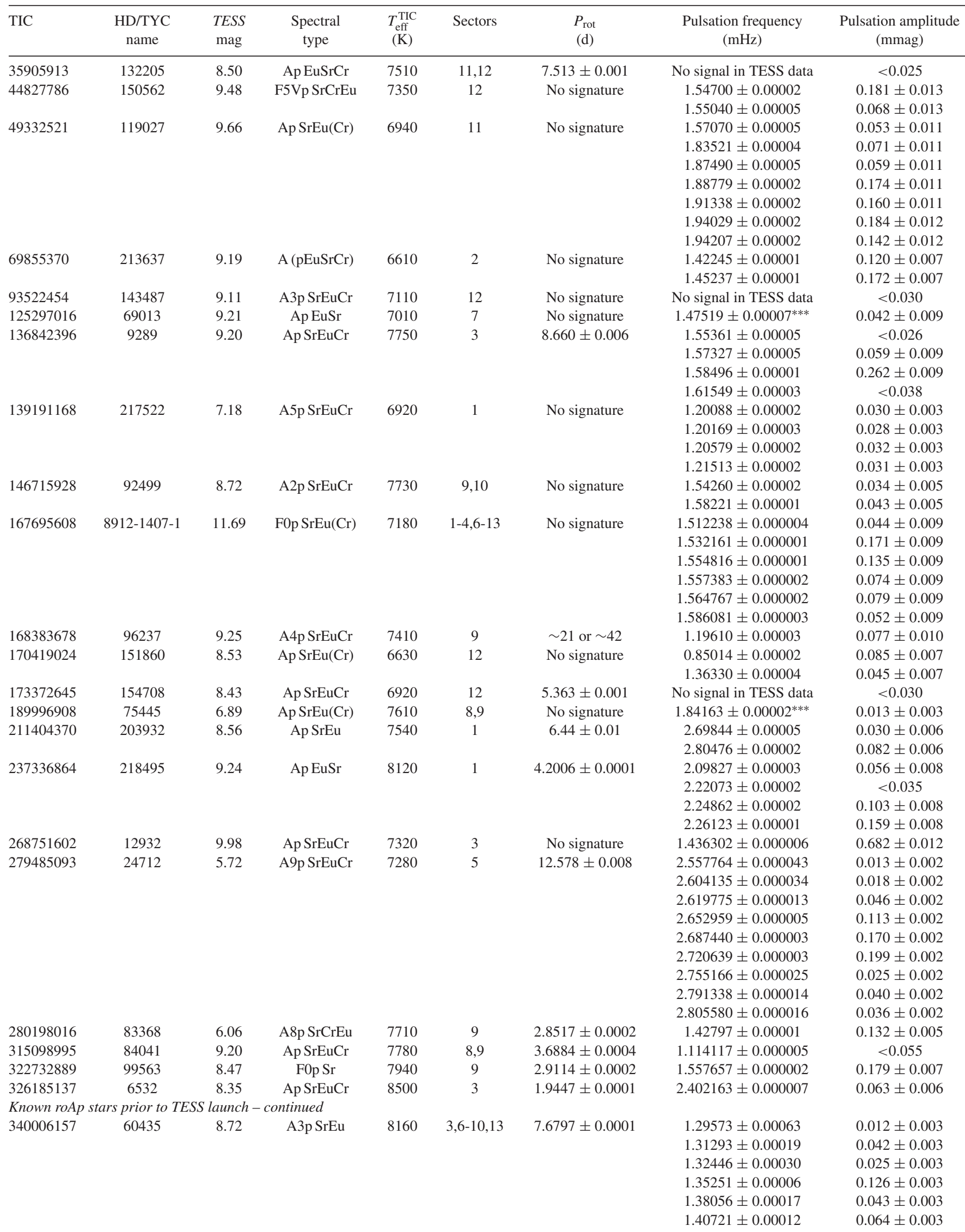


Table 1 - continued

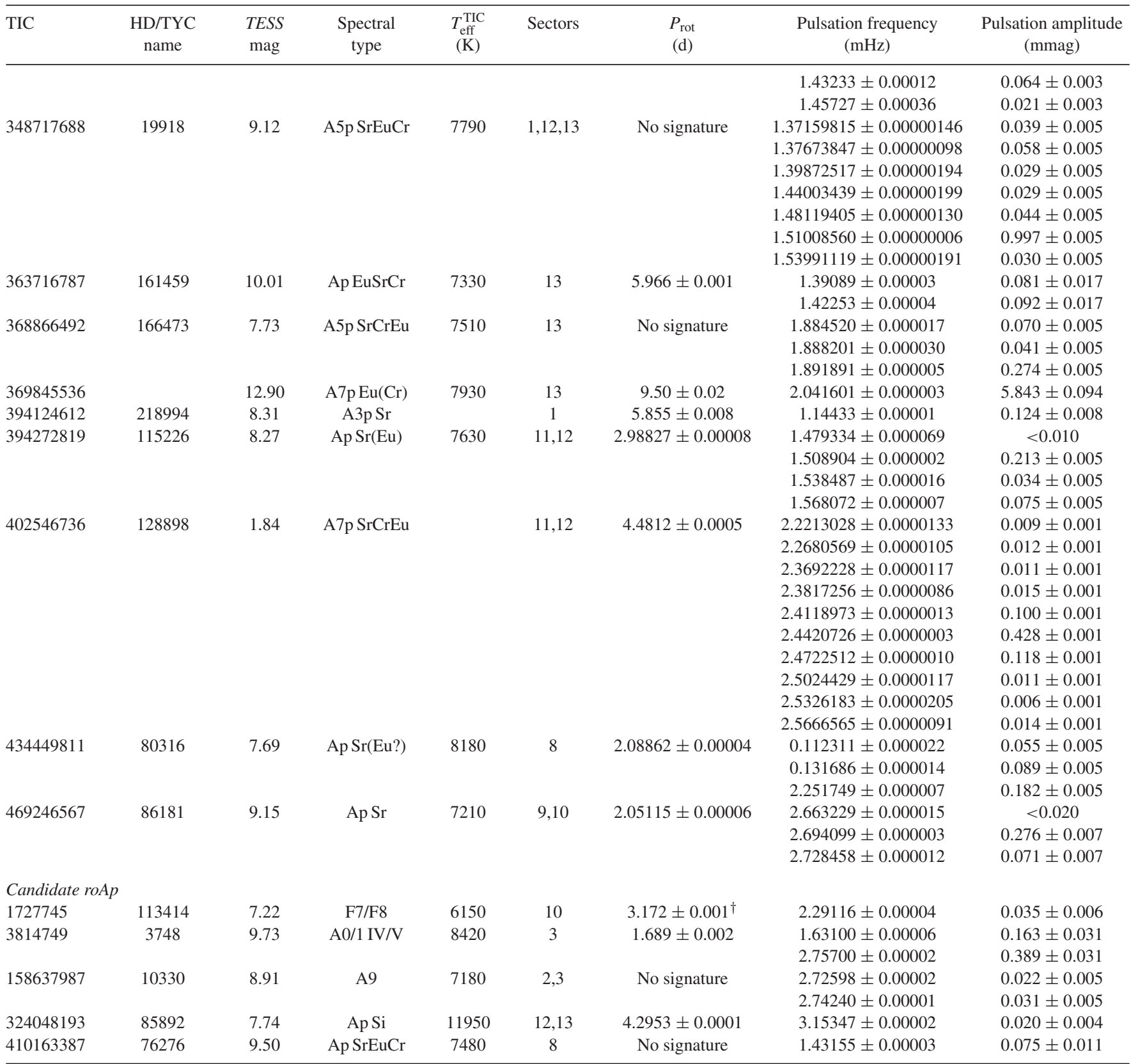

iterative pre-whitening of the light curve to a noise level determined in the range $2.3-3.3 \mathrm{mHz}\left(200-300 \mathrm{~d}^{-1}\right)$. This serves to make the noise in the amplitude spectrum white when considering the errors on pulsation signals. Any pulsation signals found were extracted through non-linear least-squares fitting. Both the derived rotation period and the pulsation frequency(ies) for each star are listed in Table 1. We provide only the presumed pulsation mode frequencies and not rotationally split sidelobes; any sidelobes detected are mentioned in the text, and indicated in the appropriate figure.

Since the launch of TESS, there have been two papers addressing the discovery of roAp stars in the new data set. The TESS Ap first light paper (Cunha et al. 2019) focused on the analysis of the first and second TESS sectors, while a paper by Balona et al. (2019) analysed data from sectors 1-7. We do not present an in depth analysis of the stars addressed in those papers again, unless there are more data available, or significant differences in the results were found. We do, however, include these stars in Table 1 to provide a complete inventory of TESS observations of roAp stars in Cycle 1.

\subsection{New TESS roAp stars}

We classify the following stars as new roAp stars based on the presence of high frequency pulsation in the target and either an Ap spectral classification, the presence of multiplets in the amplitude spectrum split by the stellar rotation frequency, or Strömgren-Crawford indices that indicate a chemically peculiar star. 

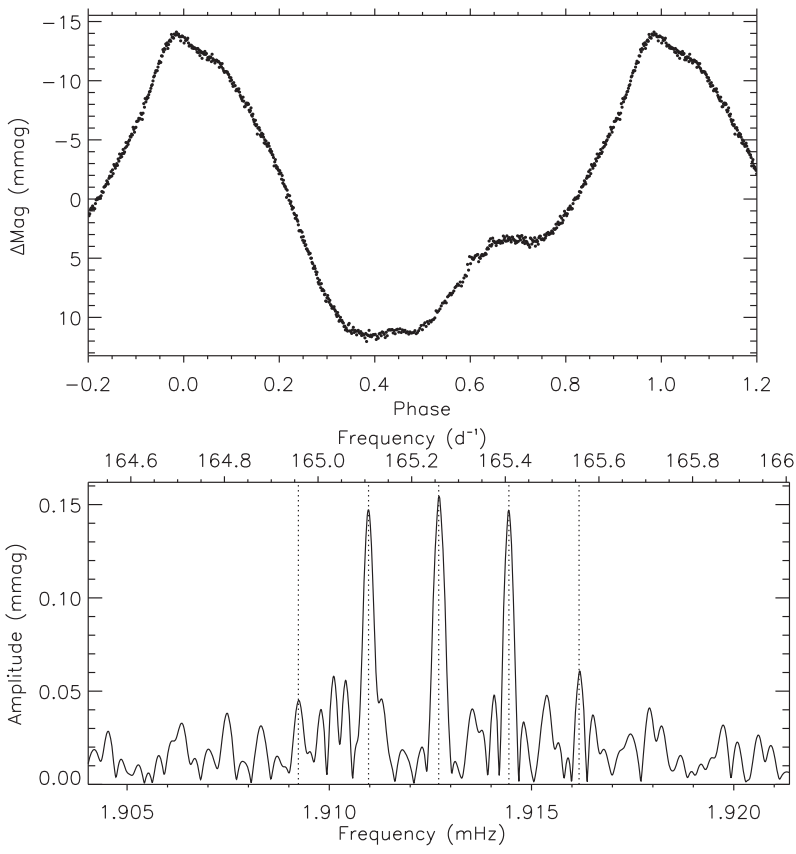

Figure 1. Top: light curve of TIC 96315731 phase folded on the derived rotation period of $6.67134 \mathrm{~d}$. Bottom: the pulsation quintuple arising from a distorted quadrupole mode. The vertical dotted lines indicate the quintuplet components.

\subsubsection{TIC 96315731}

TIC 96315731 (HD 51203) is identified in the literature as an $\alpha^{2} \mathrm{CVn}$ with a spectral type of Ap SrEuCr (Houk 1982). The star is reported to have a mean magnetic field modulus of $7.9 \pm 0.5 \mathrm{kG}$ (Chojnowski et al. 2019).

TIC 96315731 was observed by TESS during sectors 6 and 7, and shows clear signatures of rotation and pulsation, making this the first report that TIC 96315731 is a new roAp star, although Kobzar (2020) listed it as a candidate roAp star. From the combined TESS light curve, we measure a rotation period of $6.6713 \pm 0.0007 \mathrm{~d}$ which is similar to the value of $6.675 \mathrm{~d}$ presented by Netopil et al. (2017). We show a light curve phased on the rotation period in the top panel of Fig. 1.

At high frequency, the star shows an obvious pulsation signal at $1.91271 \pm 0.00001 \mathrm{mHz}\left(165.258 \pm 0.001 \mathrm{~d}^{-1}\right)$ which is flanked by two sidelobes with significant amplitude $(\mathrm{S} / \mathrm{N} \sim 14)$ separated from the pulsation by the rotation frequency, and two further sidelobes which have lower significance $(\mathrm{S} / \mathrm{N} \sim 6)$ separated by twice the rotation frequency. This is indicative of either a distorted dipole mode, or a quadrupole mode, although an analysis of the pulsation amplitude and phase as a function of rotation phase favours a distorted quadrupole mode. In the case of a distorted quadrupole mode, the pulsation phase does not change by the expected $\pi$ rad when a pulsation node crosses the line of sight, but rather results in a phase blip (see e.g. Holdsworth et al. 2016, 2018b; Shi et al. submitted). These peaks are shown in the bottom panel of Fig. 1.

\subsubsection{TIC 119327278}

TIC 119327278 (HD 45698) was initially classified as an Ap star by Bidelman \& MacConnell (1973) due to the presence of Sr absorption,
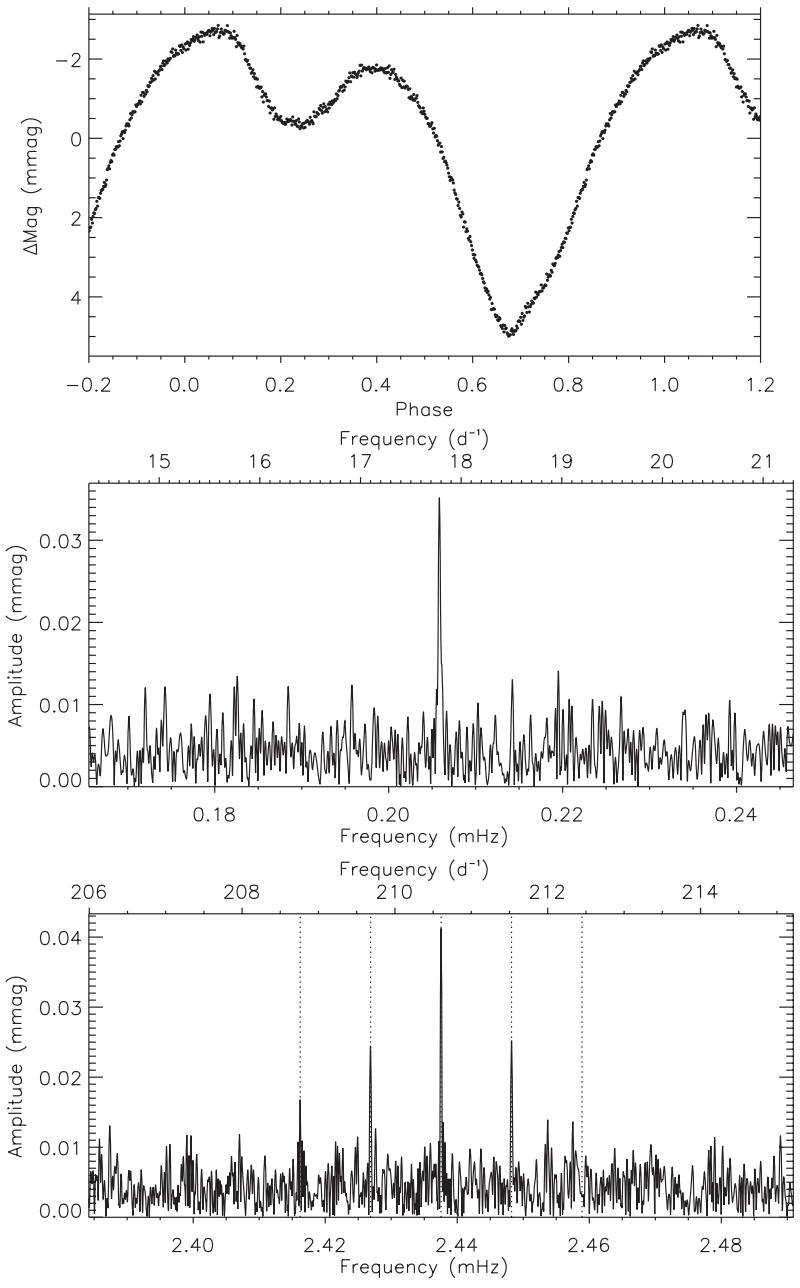

Figure 2. Top: light curve of TIC 119327278 phase folded on the derived rotation period of $1.08457 \mathrm{~d}$. Middle: view of the $\delta$ Sct mode in this star. Bottom: the pulsation multiplet signature. The vertical dotted lines indicate the quintuplet components.

with a revised spectral type of A2 SrEu provided by Renson, Gerbaldi \& Catalano (1991). There is little information about this star in the literature other than a rotation period of $1.085 \mathrm{~d}$ (Netopil et al. 2017), and a lack of pulsation signal reported by Joshi et al. (2016).

TIC 119327278 was observed during sectors 6 and 7. The data show the star to have a rotation period of $1.08457 \pm 0.00003 \mathrm{~d}$ (Fig. 2) which is in agreement with the literature. Further to the harmonic series, we identify another mode that is typical of the $\delta$ Sct stars, at a frequency of $0.20583 \pm 0.00001 \mathrm{mHz}\left(17.783 \pm 0.001 \mathrm{~d}^{-1}\right)$. Although it was not expected that Ap stars show these low-overtone modes as a result of magnetic suppression (Saio 2005), Murphy et al. (2020) have recently shown the existence of $\delta$ Sct and roAp pulsations in the same star.

The amplitude spectrum at high frequency shows a multiplet of 4 significant components $\left(v+2 v_{\text {rot }}\right.$ is in the noise) of a presumed quintuplet (Fig. 2), with the highest peak representing the pulsation mode at a frequency of $2.43755 \pm 0.00001 \mathrm{mHz}\left(210.605 \pm 0.001 \mathrm{~d}^{-1}\right)$, which was also identified by Kobzar (2020). The components are split by the rotation frequency of the star as a result of oblique pulsation, thus confirming this star as a new roAp star. Given the presence of the low-overtone mode in TIC 119327278, it may be the second example of a $\delta$ Sct-roAp hybrid star. Given the low amplitude of the 

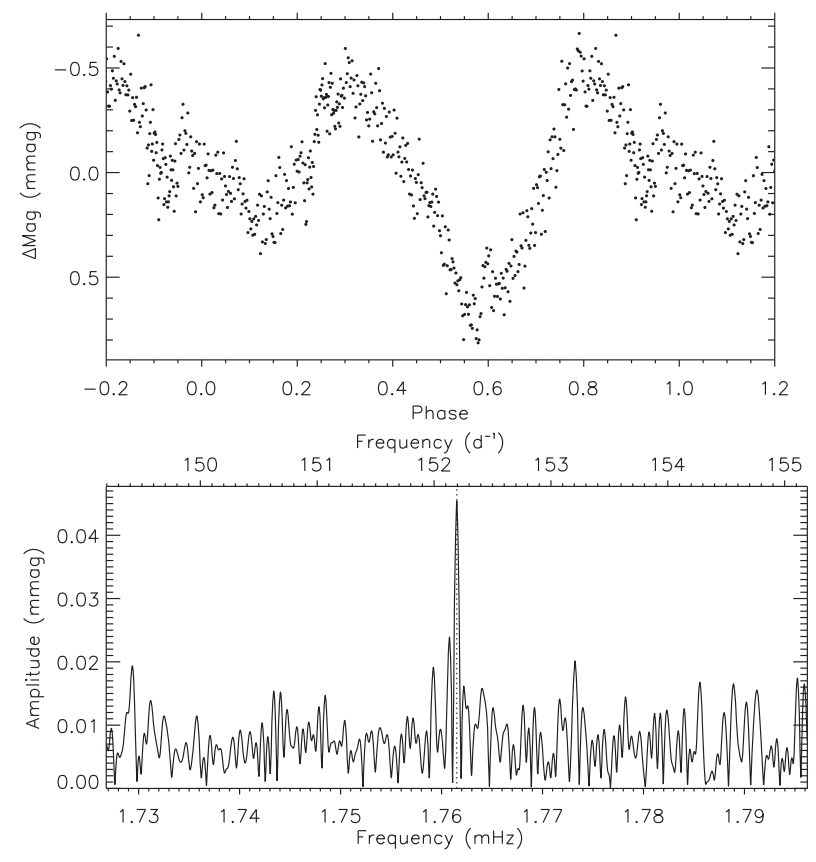

Figure 3. Top: the light curve of TIC 170586794 phased on a period of $10.305 \mathrm{~d}$. Bottom: amplitude spectrum of the light curve showing a single pulsation mode.

pulsation mode and the relatively short rotation period, analysing the pulsation amplitude and phase over the rotation period results in inconclusive results with regards to the identification of the mode degree. A detailed study, beyond the scope of this work, is needed to resolve this problem.

\subsubsection{TIC 170586794}

TIC 170586794 (HD 107619) was classified as a possible metallicline late-A type star by Houk (1982). The star was later defined to be an F0 type by Cannon \& Pickering (1993) and an F0 type $\delta$ Del star by Perry (1991). It is not a frequently studied object with the literature being mainly concerned with $u v b y \mathrm{H} \beta^{3}$ photometry. Among these studies, Perry \& Christodoulou (1996) gave dereddened Strömgren colour indexes and metallicity of the star, while Perry (1991) provided $(b-y), m_{1}, c_{1}$ colour indexes and $\mathrm{H} \beta$ index of $0.303,0.226,0.432$, and 2.682, respectively. The temperature of the star was derived as $T_{\text {eff }}=6504 \mathrm{~K}$ by Stevens, Stassun \& Gaudi (2017) and $T_{\text {eff }}=6564 \mathrm{~K}$ by Ammons et al. (2006) from photometric investigations.

TESS observed TIC 170586794 during sector 10. The light curve shows the star to have a rotation period of $10.305 \pm 0.041 \mathrm{~d}$, which has a double wave nature (Fig. 3). At high frequency, this star shows a single pulsation mode at $1.76151 \pm 0.00003 \mathrm{mHz}$ (152.194 $\pm 0.003 \mathrm{~d}^{-1}$; Fig. 3). With both the presence of a rotationally modulated light curve, and a high frequency pulsation, we revisited this star spectroscopically. Our SAAO classification spectrum (Fig. A3) shows this star to have overabundances of Eu II and $\mathrm{Cr}$ II, thus making it chemically peculiar. The Balmer lines in the spectrum are well matched to an F5 V star, thus making this a cool

\footnotetext{
${ }^{3}$ Throughout we use the $\mathrm{H} \beta$ notation to refer to the Strömgren-Crawford index to avoid confusion between angle of magnetic obliquity which is given the symbol $\beta$.
}

F5p EuCr star, and confirming it as a new roAp star and making it one of the coolest roAp stars to date.

\subsubsection{TIC 176516923}

TIC 176516923 (HD 38823) has several classifications in the literature: A5 SrEu (Vogt \& Faundez 1979; Hensberge et al. 1981); a strongly magnetic A5p Sr star (Chen, Liu \& Shan 2017); and A5 SrEuCr (Renson \& Manfroid 2009). Sikora et al. (2019a) confirmed the star as a magnetic CP star and provided several abundance measures and estimated the age to be $\log t=8.58_{-0.76}^{+0.56}$ (Gyr). Estimates of the effective temperature range from 6600 to $7700 \mathrm{~K}$, the latter of which agrees with the TIC temperature provided in Table 1. $\log g$ values range from about $3.7\left(\mathrm{~cm} \mathrm{~s}^{-2}\right)$ to $4.6\left(\mathrm{~cm} \mathrm{~s}^{-2}\right)$, with most rotational velocity measurements suggesting a $v \sin i \approx 22 \mathrm{~km} \mathrm{~s}^{-1}$.

There are several studies related to the magnetic structure of TIC 176516923. The strong magnetic field of the star was first discovered by Romanyuk et al. (2016) after three observations made in 2009 , with a $\left\langle B_{z}\right\rangle$ value of $1.74 \mathrm{kG}$. They found a variable radial velocity within a small interval around $-10 \mathrm{~km} \mathrm{~s}^{-1}$ which is significantly different from the result of $1.40 \mathrm{~km} \mathrm{~s}^{-1}$ by Grenier et al. (1999). Based on this result, they concluded that such differences in radial velocity could be an indication of a variability on a scale of up to several years or a decade. Kudryavtsev et al. (2007) performed further spectroscopic observations and calculated radial velocities ranging from -5 to $-11 \mathrm{~km} \mathrm{~s}^{-1}$. Therefore, they suspected that the star is in a binary system.

The rotation period of the star has been under debate for several years, with fits to both photometric and magnetic data being used to determine it (Hensberge et al. 1981; Kudryavtsev et al. 2006; Kudryavtsev \& Romanyuk 2012). Most recently, Bernhard, Hümmerich \& Paunzen (2020) calculated the period of the star as $8.677 \pm 0.002 \mathrm{~d}$ which is representative of the other periods reported.

TIC 176516923 was observed by TESS in sector 6 only. The data for this star provides an example where the SPOC pipeline has interfered with the astrophysical signal of the rotation signature, and injected noise into the light curve. We demonstrate this in Fig. 4 where the top panel compares the PDC_SAP (grey) data to the SAP data (black). Using the SAP data, we extract a rotation period of $8.782 \pm 0.003 \mathrm{~d}$.

The pulsation spectrum of this star consists of a quadrupole quintuplet with a missing central component, a dipole triplet also with a missing central component, and a further dipole triplet with a significant central component. Furthermore, there are two peaks with significant amplitude (labelled by the arrows in Fig. 4) that do not show any multiplet components. The pulsation modes are found at $1.76019 \pm 0.00005 \mathrm{mHz}\left(152.080 \pm 0.004 \mathrm{~d}^{-1}\right)$, $1.85820 \pm 0.00004 \mathrm{mHz} \quad\left(160.548 \pm 0.003 \mathrm{~d}^{-1}\right)$, and $1.91302 \pm 0.00005 \mathrm{mHz}\left(165.285 \pm 0.004 \mathrm{~d}^{-1}\right)$.

The difference in the multiplet structures is puzzling in this star. Although different modes have different depth pulsation cavities, one would expect both dipole modes to show very similar relative amplitudes since they are governed by the geometry of the star through $i$, the inclination angle, and $\beta$, the angle of magnetic obliquity. This difference may suggest different pulsation axes for the two dipole modes (as suggested for KIC 10195926; Kurtz et al. 2011). However, it is likely that we are seeing a depth dependence on the geometry which is being affected by different limb darkening weighting due to the stratified atmosphere of the star, as is proposed to be the case for HD 6532 (Kurtz \& Holdsworth 2020), and previously 

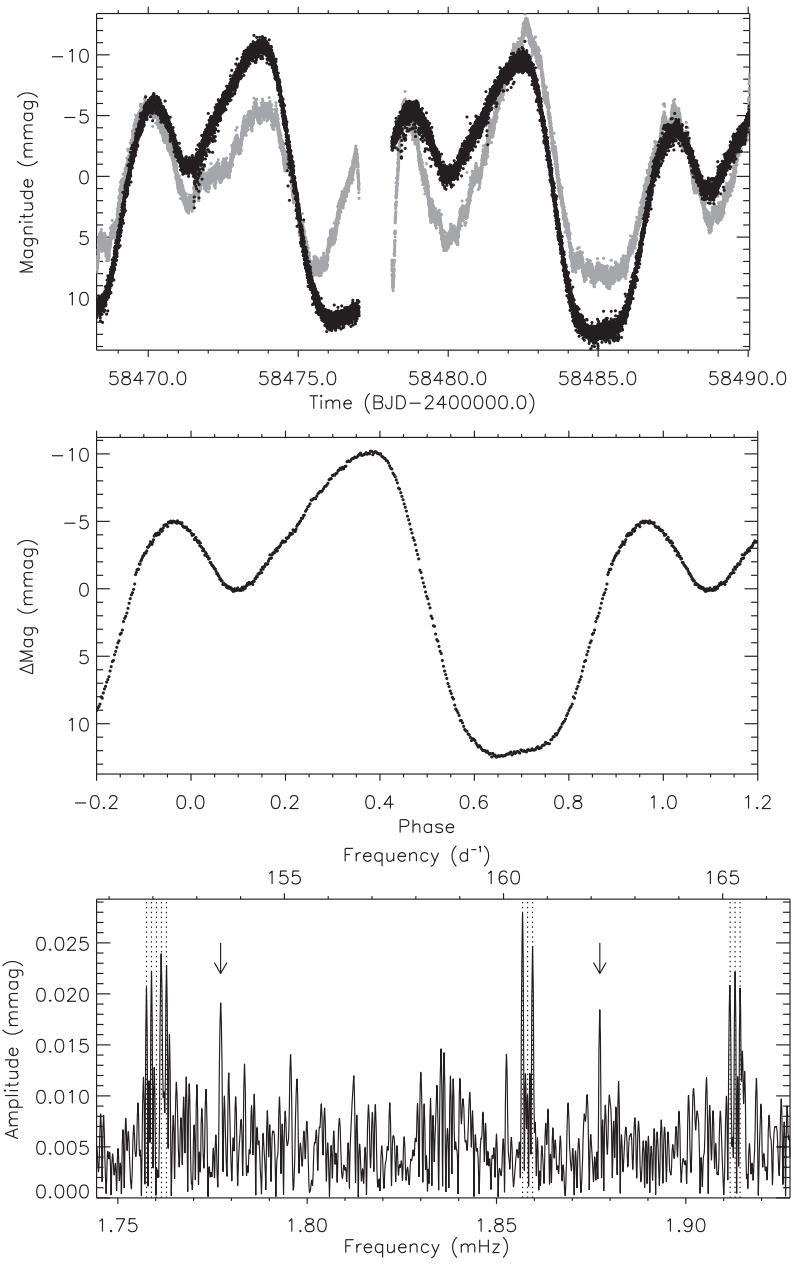

Figure 4. Top: comparison of the PDC_SAP (grey) and AP (black) light curves for TIC 176516923. Middle: SAP light curve phase folded on the derived rotation period of $8.7821 \mathrm{~d}$. Bottom: amplitude spectrum of the PDC_SAP light curve showing the pulsational variability. The vertical dotted lines indicate the multiplet components, with the arrows indicating possible further modes.

observed with time-resolved spectroscopic studies that resolved the vertical structure of pulsations by studying lines with different formation heights (Kochukhov 2006; Freyhammer et al. 2009). Another option is that the difference may result from the modes suffering different magnetic distortions in the atmosphere (Sousa \& Cunha 2011; Quitral-Manosalva, Cunha \& Kochukhov 2018), due to their slightly different frequencies. Either way, the nature of these different multiplet structures is not clear, which clearly warrants a further, in-depth, study with additional multicolour observations and detailed modelling.

\subsubsection{TIC 178575480}

TIC 178575480 (HD 55852) has a spectral classification of Ap SrEuCr (Houk \& Swift 1999a) and according to the TIC has an effective temperature of $7720 \mathrm{~K}$. Netopil et al. (2017) provide a rotation period of $4.775 \mathrm{~d}$, with Masana et al. (1998) providing Strömgren-Crawford photometric indices of $b-y=0.164, m_{1}=$ $0.237, c_{1}=0.727$ and $\mathrm{H} \beta=2.859$.
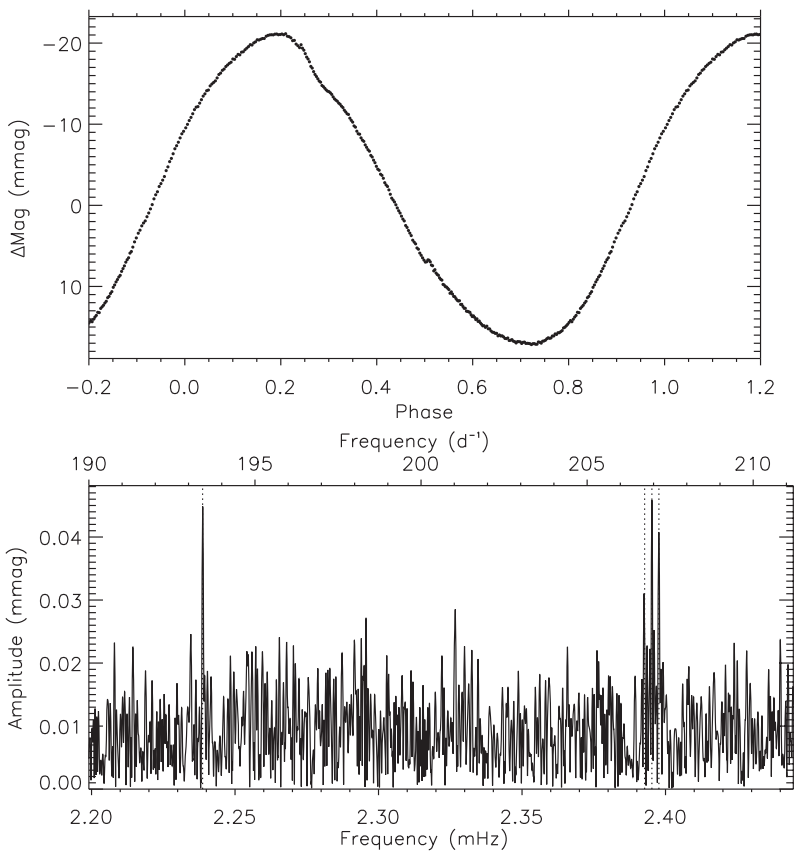

Figure 5. Top: the light curve of TIC 178575480 phase folded on the derived rotation period of $4.7788 \mathrm{~d}$. Bottom: amplitude spectrum of the light curve showing the pulsational variability. The vertical dotted lines indicate the pulsation modes and the multiplet components of the high-frequency triplet.

This star was observed in sector 7 , allowing us to measure a rotation period of $4.7788 \pm 0.0005 \mathrm{~d}$. A phase folded light curve is shown in the top panel of Fig. 5.

There are two pulsation modes seen in this star: a singlet at a frequency of $2.23870 \pm 0.00004 \mathrm{mHz}\left(193.424 \pm 0.004 \mathrm{~d}^{-1}\right)$, and a triplet with a central frequency of $2.39524 \pm 0.00004 \mathrm{mHz}$ $\left(206.949 \pm 0.003 \mathrm{~d}^{-1}\right)$. Since the two modes show different multiplet structures, it is logical to assume that the modes are of different degree, however their identification is unclear. Given that the large frequency separation in roAp stars ranges between $\sim 30-100 \mu \mathrm{Hz}$, the separation of the two modes, $\approx 156.5 \mu \mathrm{Hz}$, is plausibly 1.5 times the large frequency separation. If this is the case, the two observed modes would be of different degree, as the different structures suggest. If the separation is twice the large frequency separation, the two modes seen would be of the same degree, with the same structure. We therefore suggest the large frequency separation in this star is about $100 \mu \mathrm{Hz}$, but note modelling of this star are required to confirm this.

\subsubsection{TIC 294266638}

TIC 294266638 (TYC6021-415-1) is a star with no mention in the literature, other than a Gaia parallax measurement of $3.018 \pm 0.017$ mas, implying a distance of $331 \pm 2 \mathrm{pc}$ (Gaia Collaboration 2016, 2021).

We obtained a spectrum for this star with the SpUpNIC instrument to confirm its nature as an Ap star (Fig. A4). The spectrum shows enhancements of $\mathrm{Sr}$ and Eu leading to a classification of $\mathrm{A} 7 \mathrm{p} \mathrm{SrEu}$. There are signs of $\mathrm{Cr}$ in the spectrum at $4111 \AA$, but no other lines of this element are clearly enhanced.

This star was observed in sector 8. There is no evidence of rotational variability in the TESS light curve, however there are clear pulsation signals, as shown in Fig. 6. 

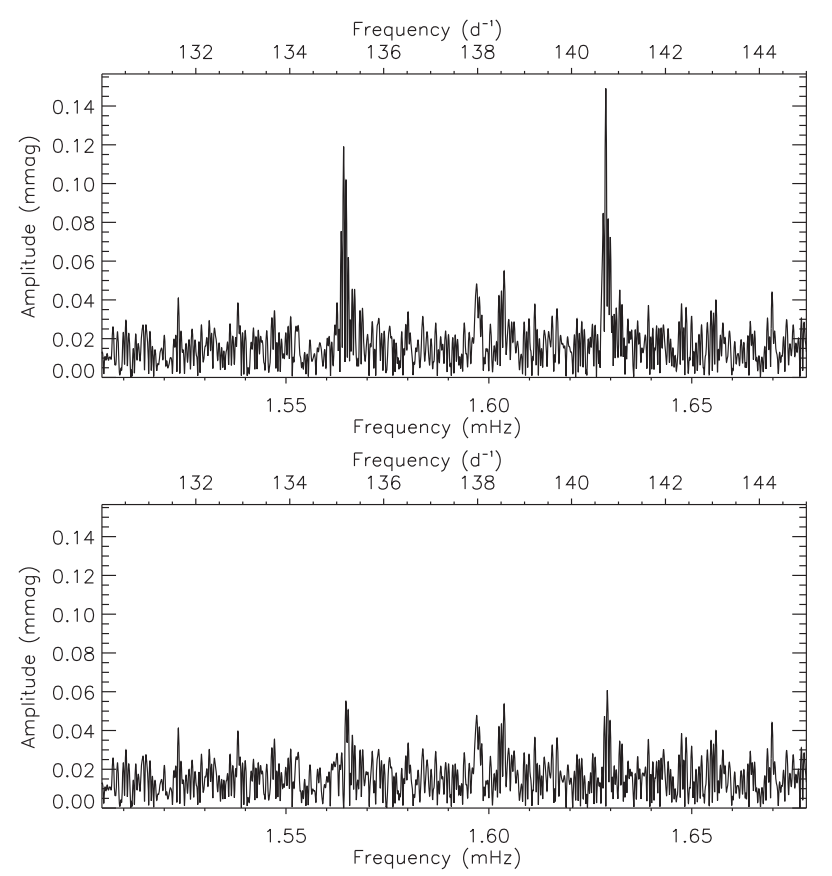

Figure 6. Top: pulsation modes in TIC 294266638. Bottom: after removing the obvious pulsations, excess power is remaining which is suggestive of frequency variability.

We extract two pulsation modes from the amplitude spectrum, at $1.56421 \pm 0.00002 \mathrm{mHz}\left(135.148 \pm 0.002 \mathrm{~d}^{-1}\right)$ and $1.62882 \pm 0.00002 \mathrm{mHz}\left(140.730 \pm 0.002 \mathrm{~d}^{-1}\right)$. The separation of these two modes, $65 \mu \mathrm{Hz}$, is plausibly the large frequency separation for this star. After pre-whitening these peaks, there is still evidence of excess power in the residual spectrum. This is indicative of phase/amplitude variability (e.g. Holdsworth 2021). Furthermore, there is evidence of two additional modes at low amplitude around $1.6 \mathrm{mHz}$ which are significant. However, given the broad nature of the peaks, a clear frequency value cannot be determined.

\subsubsection{TIC 294769049}

TIC 294769049 (HD 161423) was classified as Ap SrEu(Cr) by Houk \& Cowley (1975), and listed in the catalogue of Renson \& Manfroid (2009) with a spectral type of A2 SrEu. TIC 294769049 has been the target in two searches for rapid variability; both Martinez \& Kurtz (1994b) and Joshi et al. (2016) returned null results.

TESS observed this star during sectors 12 and 13, providing a light curve with a time base of $57.4 \mathrm{~d}$. From this we measure a rotation period of $10.4641 \pm 0.0007 \mathrm{~d}$, and show a phase folded light curve in Fig. 7. Analysis of the light curve at high frequency shows a single pulsation mode at $2.26044 \pm 0.00001 \mathrm{mHz}\left(195.302 \pm 0.001 \mathrm{~d}^{-1}\right.$; Fig. 7). The lack of an obliquely split multiplet suggests that either the pulsation axis is aligned with the rotation axis (thus $\beta=0$, assuming the original oblique pulsator model), despite the significant rotational variation, or the mode is an undistorted radial mode. The former would imply that the spots causing the mean light variation are not close to the pulsation pole (Kochukhov et al. 2004; Alecian $\&$ Stift 2010), or that the pulsation axis is aligned with the rotation axis, rather than the magnetic axis (Bigot \& Dziembowski 2002).

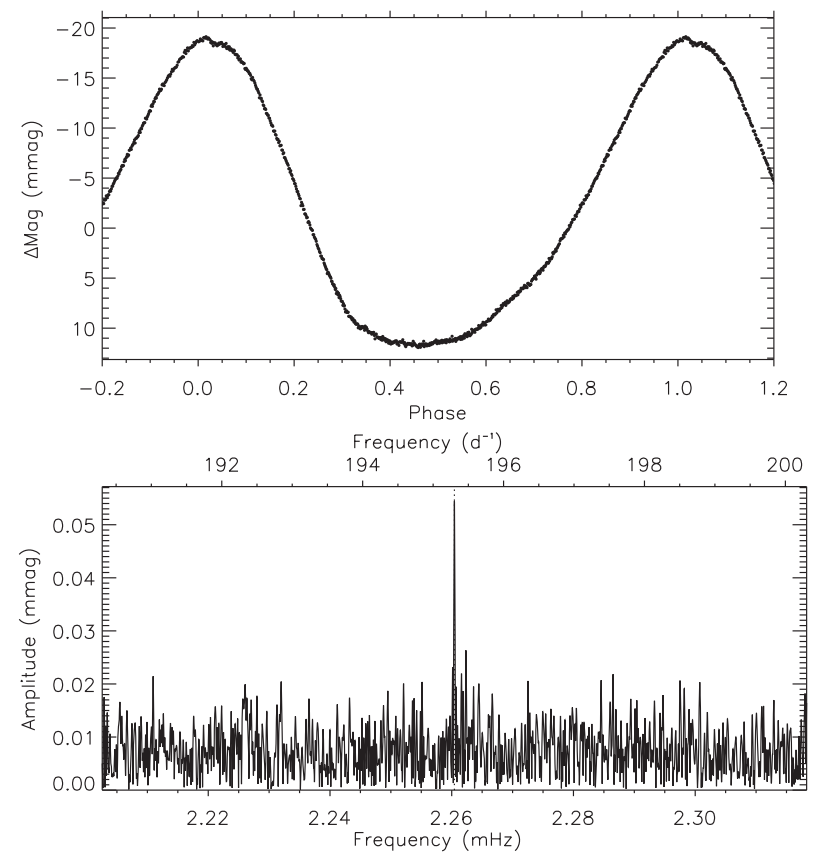

Figure 7. Top: phase folded light curve of TIC 294769049, folded on a period of $10.4641 \mathrm{~d}$. Bottom: amplitude spectrum showing the only pulsation mode in this star, and the lack of a rotationally split multiplet.

\subsubsection{TIC 310817678}

TIC 310817678 (HD 88507) was classified as Ap SrEu(Cr) by Houk (1978) and in the catalogue of Renson \& Manfroid (2009) it is quoted as an ApSrEu star. There is only one evaluation of the atmospheric parameters given by the TIC: $T_{\text {eff }}=8230 \mathrm{~K}$ and $\log g$ $=4.08\left(\mathrm{~cm} \mathrm{~s}^{-2}\right)$.

TESS observed this star in sectors 9 and 10, providing a $51.8 \mathrm{~d}$ time base. We measure a rotation period of $2.75003 \pm 0.00008 \mathrm{~d}$ (Fig. 8). The pulsation spectrum in this star is rich, consisting of a dipole triplet centred on $1.17098 \pm 0.00002 \mathrm{mHz}\left(101.173 \pm 0.002 \mathrm{~d}^{-1}\right)$, a quadrupole quintuplet with missing $v_{\text {rot }}$ sidelobes centred on $1.205272 \pm 0.000003 \mathrm{mHz}\left(104.1355 \pm 0.0002 \mathrm{~d}^{-1}\right)$, and another dipole with a central frequency at $1.22989 \pm 0.00003 \mathrm{mHz}$ $\left(106.263 \pm 0.003 \mathrm{~d}^{-1}\right)$. The two dipole modes are separated by $58.9 \mu \mathrm{Hz}$ which is plausibly the large frequency separation for this star. Kobzar (2020) identified this star as a candidate roAp star.

The high-frequency dipole sidelobes are unlike those seen in the other multiplets: they form doublets. We suspect that this is a signature of magnetic distortion since despite the sidelobes having greater amplitude than the low-frequency dipole mode, the central component is entirely absent. This star is another example of how the multiplet structures vary for the same degree mode.

\subsubsection{TIC 356088697}

TIC 356088697 (HD 76460) was classified as an Ap Sr by Houk \& Cowley (1975) with Elkin, Kurtz \& Nitschelm (2012) measuring a magnetic field strength of $3.6 \mathrm{kG}$ through partial Zeeman splitting of Fe lines. The authors also determined the effective temperature to be $7200 \mathrm{~K}$ and $v \sin i=3 \mathrm{~km} \mathrm{~s}^{-1}$, although this value is an upper limit due to the resolution limit of their data.

Observed in three consecutive TESS sectors (9-11), this star shows no signature of rotation. To confirm its Ap nature, we obtained a 

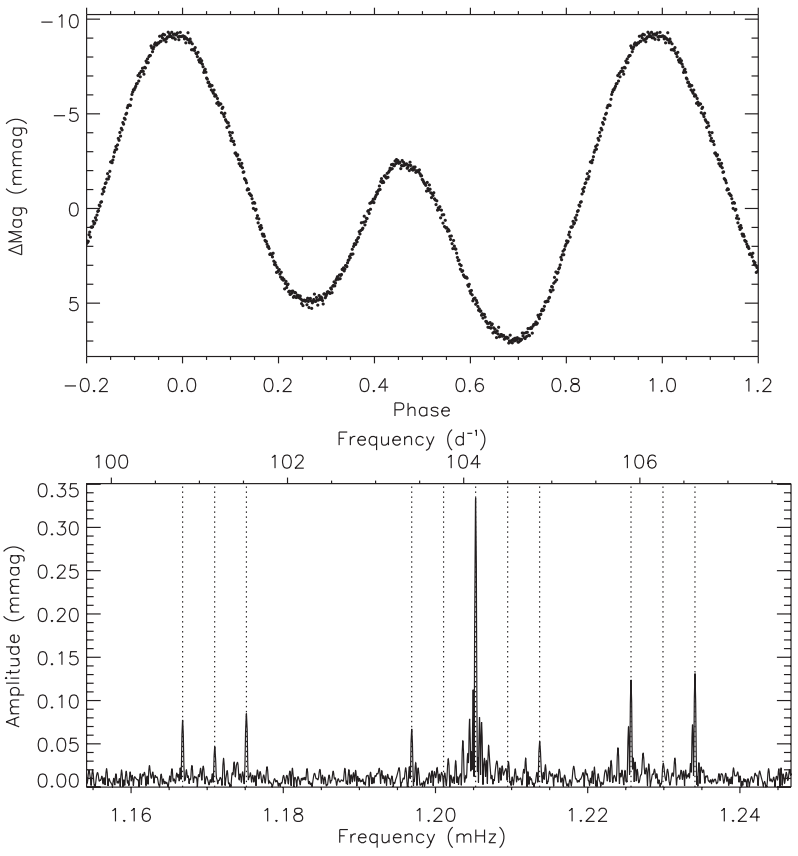

Figure 8. Top: phase folded light curve of TIC 310817678, folded on a rotation period of $2.75003 \mathrm{~d}$. Bottom: amplitude spectrum showing the rich pulsation pattern in this star. The vertical dotted lines denote the frequencies of rotationally split multiplet components.

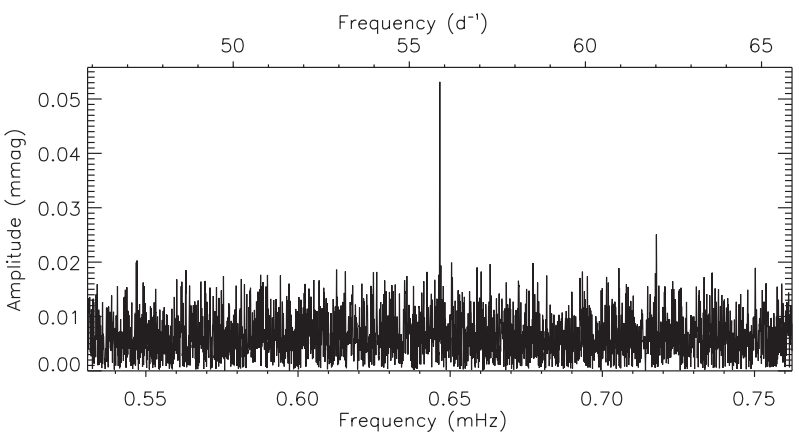

Figure 9. Amplitude spectrum of TIC 356088697 showing the single pulsation mode in this star.

spectrum with SpUpNIC and derived a spectral class of A8p SrEuCr (Fig. A5).

A single pulsation signal is found in this star at a frequency of $0.646584 \pm 0.000008 \mathrm{mHz}\left(55.8649 \pm 0.0007 \mathrm{~d}^{-1}\right)$ with an amplitude of $0.053 \pm 0.006 \mathrm{mmag}$ (Fig. 9), which was also noted by Mathys et al. (2020b). This mode does not show any amplitude or phase variability over the observing window, which would be consistent with a rotation period much longer than the observing window (Mathys et al. 2020b). The single mode in this star is at a frequency commonly associated with the $\delta$ Sct stars (Bowman \& Kurtz 2018), but it is uncommon to find just a single frequency. Therefore, with just a single mode and an Ap stellar classification, this star can be classed as an roAp star, making it the longest period roAp star, after HD 177765, which was discovered by Alentiev et al. (2012) with the mode frequency later refined to be $0.702580 \pm 0.000006 \mathrm{mHz}$ $\left(60.7029 \pm 0.0005 \mathrm{~d}^{-1}\right.$; Holdsworth 2016). Given that the mode frequency overlaps with the $\delta$ Sct frequency range, modelling of the pulsation is needed to confirm the overtone is representative of an roAp pulsation.
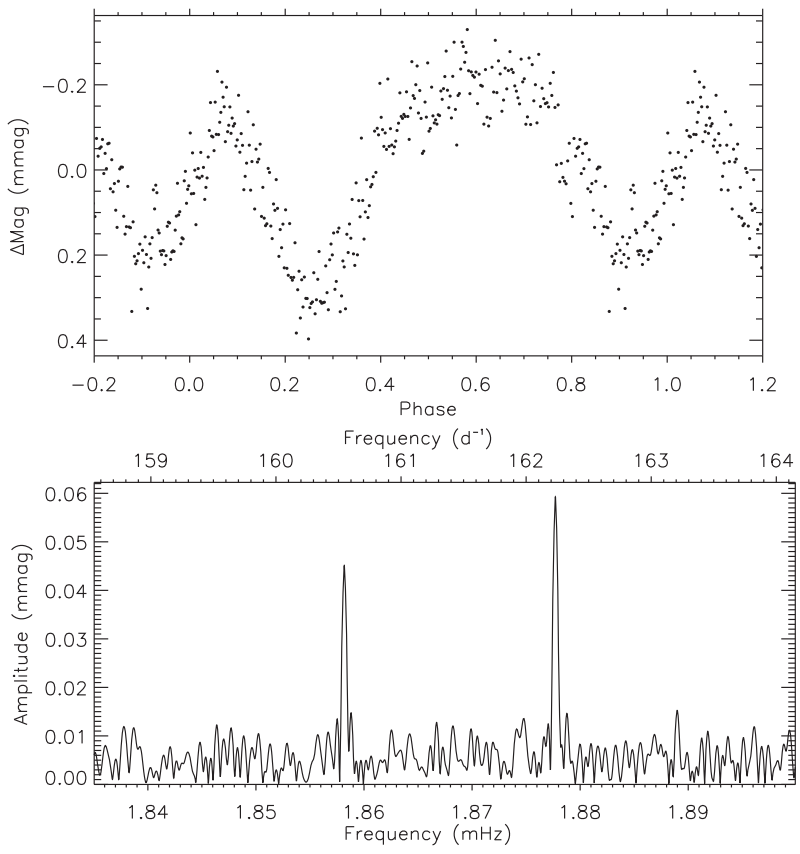

Figure 10. Top: phase folded light curve on the assumed rotation period of $4.189 \mathrm{~d}$. The significant scatter is a result of a second significant low frequency. Bottom: amplitude spectrum of TIC 380651050 showing the two pulsation modes in this star.

\subsubsection{TIC 380651050}

TIC 380651050 (HD 176384) has no in-depth studies in the literature. Reiners \& Zechmeister (2020) provide a mass of $1.25 \mathrm{M}_{\odot}$ and $T_{\text {eff }}=6531 \mathrm{~K}$, with Paunzen (2015) giving Strömgren-Crawford photometric indices of $b-y=0.285, m_{1}=0.162, c_{1}=0.446$, and $\mathrm{H} \beta=2.679$, which are consistent with a cool Ap star.

TIC 380651050 was observed in sector 13. There are two harmonic series at low frequency which both give plausible rotation periods; they are: $4.19 \pm 0.01 \mathrm{~d}$ and $3.51 \pm 0.02 \mathrm{~d}$. We take the highest amplitude signal to be the rotation frequency (Fig. 10), but suggest further observations are obtained to corroborate this. One of the two periods may be the result of binarity or contamination by a background star; spectroscopic observations will provide insight into this.

Two high frequency pulsation modes are found in this star: $1.85817 \pm 0.00002 \mathrm{mHz}\left(160.546 \pm 0.002 \mathrm{~d}^{-1}\right)$ and $1.87771 \pm 0.00002 \mathrm{mHz}\left(162.234 \pm 0.002 \mathrm{~d}^{-1}\right)$ which are separated by $19.53 \pm 0.03 \mu \mathrm{Hz}$ (Fig. 10). This separation is plausibly half of the large frequency separation, and would imply that the star is quite evolved, possibly beyond the main sequence. This star was also identified in Kobzar (2020) as a candidate roAp star.

\subsubsection{TIC 387115314}

TIC 387115314 (TYC 9462-347-1) has a spectral classification of A5 (Jackson \& Stoy 1954), and has atmospheric parameters $T_{\text {eff }}=$ $7765 \mathrm{~K}$, surface gravity $\log g=4.08\left(\mathrm{~cm} \mathrm{~s}^{-2}\right)$, and metallicity $[\mathrm{M} / \mathrm{H}]=0.18$ determined by Kordopatis et al. (2013) on the basis of RAdial Velocity Experiment (RAVE) data.

The star was observed in sector 13, from which we derive a rotation period of $5.264 \pm 0.002 \mathrm{~d}$ (Fig. 11). We find a rich pulsation spectrum for this star at high frequencies, also seen by Kobzar (2020). Although we have a well-determined rotation 

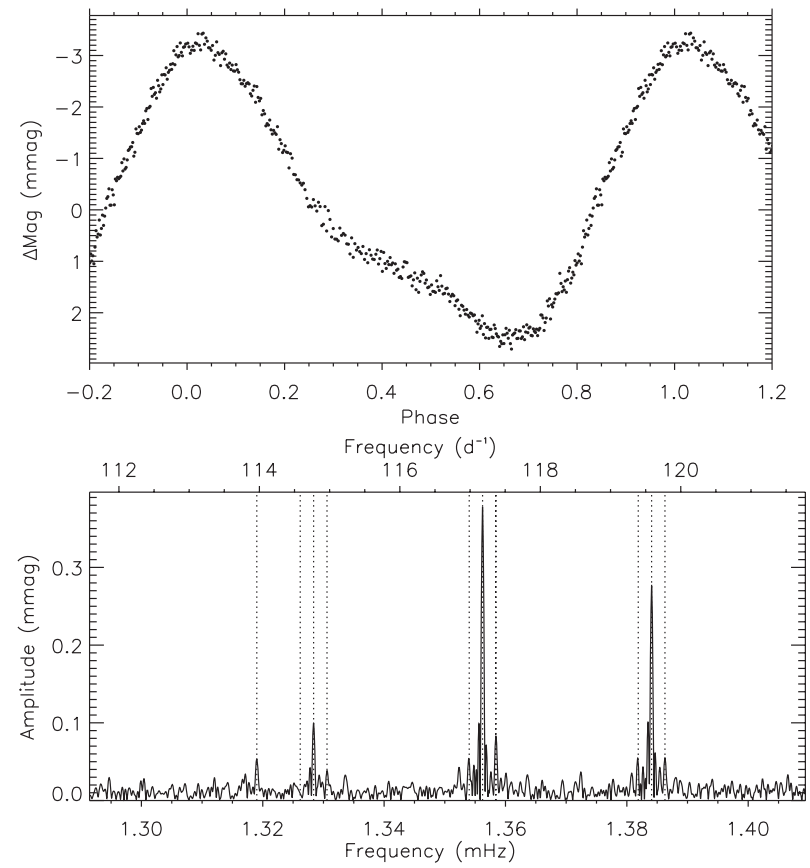

Figure 11. Top: phase folded light curve of TIC 387115314, folded on the rotation period of $5.264 \mathrm{~d}$. Bottom: amplitude spectrum showing the pulsation modes in this star.

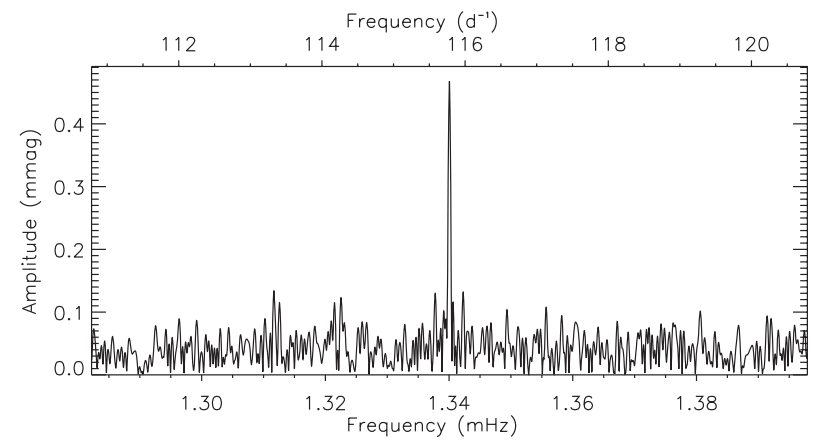

Figure 12. Amplitude spectrum showing the pulsation mode in TIC 466260580 .

period, and significant mean light variations, the modes do not show significant rotationally split sidelobes which implies we see mostly one pulsation pole over the entire rotation period. The pulsation frequencies that we identify are: a singlet at $1.31902 \pm 0.00004 \mathrm{mHz}$ $\left(113.963 \pm 0.003 \mathrm{~d}^{-1}\right)$, two components of a triplet with the mode at $1.32838 \pm 0.00002 \mathrm{mHz}\left(114.772 \pm 0.002 \mathrm{~d}^{-1}\right)$, a triplet centred on $1.356225 \pm 0.000006 \mathrm{mHz}\left(117.1778 \pm 0.0005 \mathrm{~d}^{-1}\right)$ and another triplet centred on $1.384083 \pm 0.000007 \mathrm{mHz}$ $\left(119.5848 \pm 0.0006 \mathrm{~d}^{-1}\right)$. These modes, with their multiplet components, are shown in Fig. 11.

The separation of the three highest modes is $\sim 27.85 \mu \mathrm{Hz}$, with the lowest frequency two modes separated by $9.36 \mu \mathrm{Hz}$. Since we cannot determine the degree of the modes showing multiplets, due to the low amplitude of the obliquely split sidelobes, we are unable to determine if the separation of $\sim 27.85 \mu \mathrm{Hz}$ is the large frequency separation, or half of it. The separation of $9.36 \mu \mathrm{Hz}$ is plausibly the small frequency separation for this star.

\subsubsection{TIC 466260580}

TIC 466260580 (TYC 9087-1516-1) is a relatively obscure star with no previous reports of any variability. The RAVE survey (Kunder et al. 2017) obtained $T_{\text {eff }}=6780 \pm 170 \mathrm{~K}, \log g=$ $4.03 \pm 0.27\left(\mathrm{~cm} \mathrm{~s}^{-2}\right)$ and $[\mathrm{M} / \mathrm{H}]=-0.7 \pm 0.2$ which are consistent with the TIC values, although the metallicity is strikingly low for an Ap star. These suggest the star is spectral type F3, which is consistent with the Tycho $B-V=0.40 \pm 0.07$. However, the $T_{\text {eff }}$ is uncertain; for example, McDonald, Zijlstra \& Watson (2017) obtained $T_{\text {eff }}=$ $6420 \pm 170 \mathrm{~K}$ from SED fitting, while Gaia Collaboration (2018) give a value around $7000 \mathrm{~K}$.

We have also obtained a spectrum of this star with the HRS on SALT. From this, we obtain $T_{\text {eff }}=6800 \pm 200 \mathrm{~K}, \log g=$ $4.0 \pm 0.2\left(\mathrm{~cm} \mathrm{~s}^{-2}\right)$ and $[\mathrm{Fe} / \mathrm{H}]=-0.6 \pm 0.2$, which are consistent with the RAVE results. We estimate a spectral type of Ap EuCr, and note that rare earth elements are enhanced in the region around $6150 \AA$, confirming the star to be an Ap star. A full abundance analysis is beyond the scope of this work, but is required to address the low metallicity in this star.

The TESS data, obtained in sector 13, do not show clear signs of rotational variability, but exhibit many low-frequency variations. The pulsation signal is clear in this star, at a frequency of $1.34006 \pm 0.00002 \mathrm{mHz}\left(115.781 \pm 0.002 \mathrm{~d}^{-1}\right.$; Fig. 12).

\section{2 roAp stars previously discovered by $T E S S$}

\subsubsection{TIC 12968953}

TIC 12968953 (HD 217704) star was observed in TESS sector 2 and was analysed by Cunha et al. (2019). We find no significant differences between our analysis here and that previously presented. We refer the reader to the aforementioned publication for the details on TIC 12968953. However, we obtained a new spectrum of this target. TIC 12968953 was classified as Ap Sr by Houk (1982); with our SAAO spectrum (Fig. A6), we classify this star as A7p SrEuCr, and determine a temperature of $T_{\text {eff }}=7800 \pm 200 \mathrm{~K}$ through comparison with synthetic spectra.

\subsubsection{TIC 17676722}

TIC 17676722 (HD 63773) was observed in sector 7, and reported as an roAp star by Balona et al. (2019). We present a revised rotation period of $1.5995 \pm 0.0003 \mathrm{~d}$, which was derived by fitting a 10-component harmonic series to the data. As in Balona et al. (2019) we find four significant peaks which we interpret to be a single quadrupole pulsation mode $(1.941545 \pm 0.000002 \mathrm{mHz}$; $167.7495 \pm 0.0002 \mathrm{~d}^{-1}$ ) and 3 sidelobes split by the rotation frequency of the star.

This star has been classified as A2 (Cannon \& Pickering 1993), however our SAAO classification spectrum shows the star to be A3p SrEuCr (Fig. A7).

\subsubsection{TIC 41259805}

TIC 41259805 (HD 43226) was reported by both Cunha et al. (2019) and Balona et al. (2019) to be an roAp star, based on 7 sectors of data. There are now 12 sectors of data available for this star from Cycle 1, with no sector 9 data. With this extended time base, we are able to report a more precise rotation period of $1.714489 \pm 0.000002 \mathrm{~d}$; Fig. 13). 

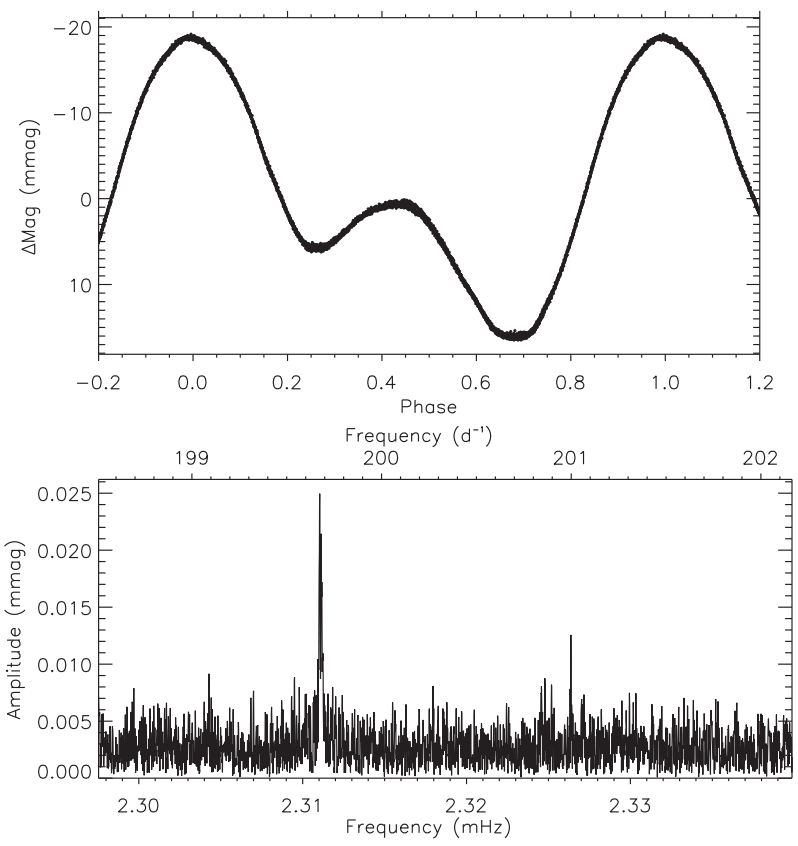

Figure 13. Top: Phase folded light curve of TIC 41259805, folded on a period of $1.714489 \mathrm{~d}$. Bottom: the two pulsation modes found in this star, with the lower frequency mode showing evidence of frequency variability.

There is clear frequency variability in this star, as seen by the ragged nature of the pulsation mode at $2.311039 \pm 0.000001 \mathrm{mHz}$ $\left(199.6738 \pm 0.0001 \mathrm{~d}^{-1}\right)$ in Fig. 13, but not in the mode at $2.326368 \pm 0.000003 \mathrm{mHz}\left(200.9982 \pm 0.0003 \mathrm{~d}^{-1}\right)$. The transient nature of the modes explains the presence of the $v-v_{\text {rot }}$ peak found by Cunha et al. (2019), and the lack of its detection by Balona et al. (2019). We note that here the amplitude is about half that reported in Cunha et al. (2019) as a result of the power being spread over a broad frequency range. Since the frequency variability is only seen in one mode, it is likely to be intrinsic to the star, rather than caused by binary motion, for example. This type of frequency variability seems common in the roAp stars studied with high-precision data (Holdsworth 2021), with a possible explanation of stochastic perturbations of classical pulsators (Avelino, Cunha \& Chaplin 2020; Cunha, Avelino \& Chaplin 2020), although this is currently untested.

We have obtained a classification spectrum of this star, since the literature class of A0 SrEu implies a hot star which is uncommon amongst the roAp stars. With our spectrum (Fig. A8) we derive a spectral class of $\mathrm{A} 6 \mathrm{pSrEu}(\mathrm{Cr})$, with Balmer and metal lines indicating an A6p star.

\subsubsection{TIC 49818005}

TIC 49818005 (HD 19687) was given the spectral type A9IV/V in the Michigan Catalog (Houk \& Swift 1999b). Conversely, Balona et al. (2019) list a spectral type of A3, which is inconsistent with the $6829 \mathrm{~K}$ temperature they cite (from Stevens et al. 2017). There is no information on its peculiarity, if any, but Balona et al. (2019) found a significant high frequency at $1.6424 \mathrm{mHz}\left(141.9 \mathrm{~d}^{-1}\right)$, suggesting this could be an roAp star. We obtained a new low-resolution classification spectrum to clarify this point (Fig. A9). We find the star to be an $\mathrm{F} 2 \mathrm{p} \mathrm{SrEu}(\mathrm{Cr})$, with Balmer and metal lines indicating an F1p star, and confirm the presence of rare earth element lines in a high-resolution SALT spectrum. With the high-resolution spectrum,

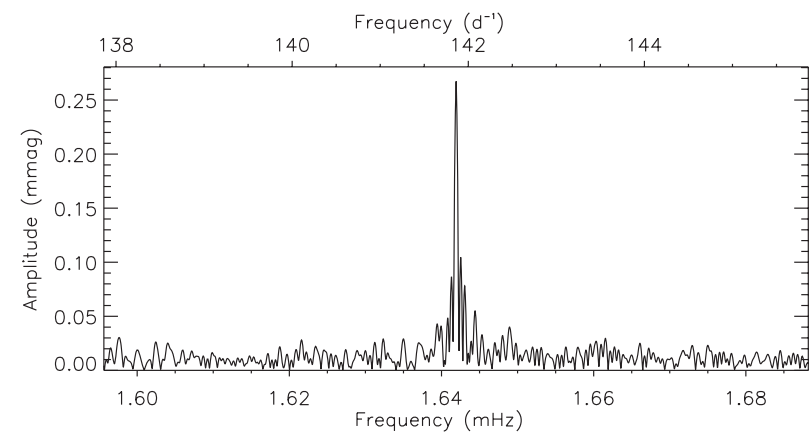

Figure 14. The pulsation mode in TIC 49818005.

we determine $T_{\text {eff }}=7100 \pm 100 \mathrm{~K}$, which is consistent with the TIC value and previous determinations.

The TESS observations of this star were collected in sector 4, which were analysed by Balona et al. (2019). We present a slightly different value for the pulsation frequency in this star, $1.641910 \pm 0.000008 \mathrm{mHz}\left(141.8619 \pm 0.0007 \mathrm{~d}^{-1}\right)$, as shown in Fig. 14. We suspect this difference is a result of the data treatment at low-frequency, which is not discussed in Balona et al. (2019).

\subsubsection{TIC 152808505}

TIC 152808505 (HD 216641) was observed in TESS sector 1 and was analysed by Cunha et al. (2019). We find no significant differences between our analysis here and that previously presented, so we refer the reader to the aforementioned publication for the details on this star, but provide the derived rotation period and pulsation frequencies in Table 1.

Since the only classification for this star is F3 IV/V (Houk 1978), we obtained a new spectrum of this target with the SpUpNIC instrument at SAAO (Fig. A10). We classify this star as F3p EuCr, and determine a temperature of $T_{\text {eff }}=6900 \pm 300 \mathrm{~K}$ through comparison with synthetic spectra. This confirms this star to be an roAp star.

\subsubsection{TIC 156886111}

TIC 156886111 (HD 47284) was observed in sectors 6 and 7, and originally reported as an roAp star by Balona et al. (2019). The spectral type of this star is commonly given in the literature as A5 SiEuCr (Houk 1978). However, we have obtained a new spectrum of this star (Fig. A11) which shows this star to be an A8p SrEuCr star (showing $\mathrm{Sr}$ rather than $\mathrm{Si}$ ).

We precisely determine the rotation period of this star to be $6.8580 \pm 0.0003 \mathrm{~d}$ through analysis of the mean light variations, which is in line with the literature value (Watson, Henden \& Price 2006). After the rotation signal, the dominant mode in this star is at $0.063251 \pm 0.000002 \mathrm{mHz}\left(5.4649 \pm 0.0002 \mathrm{~d}^{-1}\right)$. This may be another example of a $\delta$ Sct-roAp hybrid, as discussed for TIC 158637987.

We find no more pulsation modes in the data than were presented by Balona et al. (2019); we find a singlet at $1.27457 \pm 0.00002 \mathrm{mHz}$ $\left(110.123 \pm 0.002 \mathrm{~d}^{-1}\right)$, a doublet which is presumed to be a dipole triplet with a missing central component at $1.30274 \pm 0.00001 \mathrm{mHz}$ $\left(112.557 \pm 0.001 \mathrm{~d}^{-1}\right)$, and a quadrupole quintuplet centred on a frequency of $1.317816 \pm 0.000008 \mathrm{mHz}\left(113.8593 \pm 0.0007 \mathrm{~d}^{-1}\right)$. 

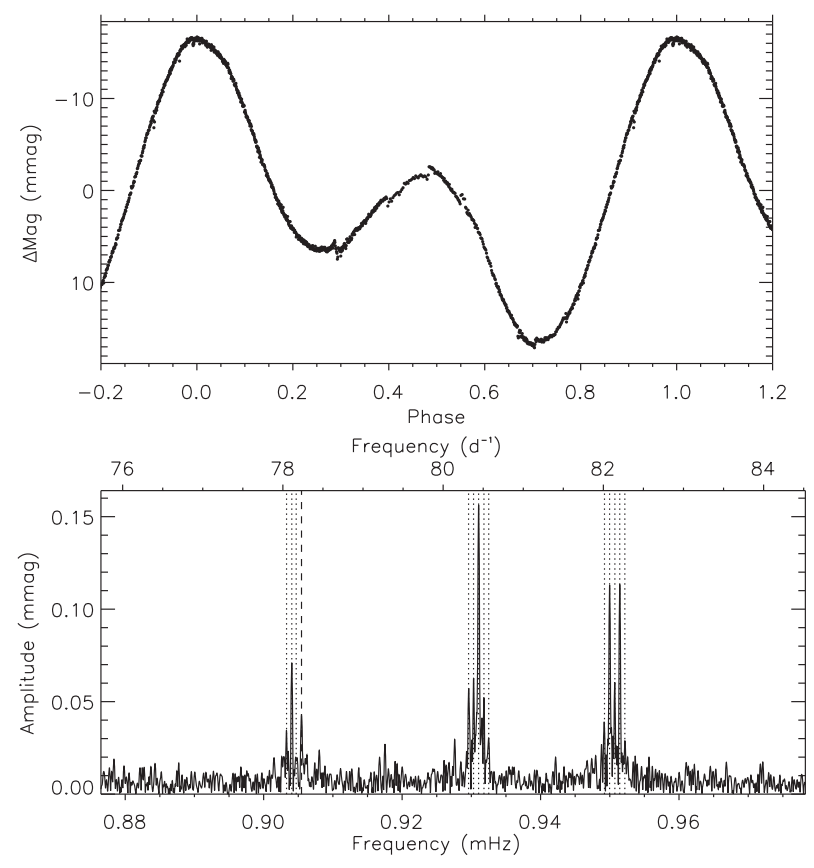

Figure 15. Top: phase folded light curve of TIC 259587315, folded on a period of $15.776 \mathrm{~d}$. Bottom: the pulsation modes in TIC 259587315 . The vertical dotted lines show the components of obliquely split multiplets, with the dashed vertical line indicating an independent mode.

\subsubsection{TIC 259587315}

TIC 259587315 (HD 30849) was classified as an Ap SrEuCr by Houk (1978), and has $T_{\text {eff }}$ measures of $7720 \mathrm{~K}$ in the TIC, $7250 \mathrm{~K}$ (Glagolevskij 1994), 8187 K (North et al. 1997), and 8000 K (Netopil et al. 2017).

The TESS data for this star, collected in sectors 4 and 5, have been analysed independently by Balona et al. (2019) and Sikora et al. (2019c) who both announced this star to be an roAp star, but provided conflicting rotational periods. Previous searches for highfrequency variability by Martinez \& Kurtz (1994b) and Hubrig et al. (2000) were unsuccessful.

Here, we use the SAP data from sector 4 (since the pipeline has distorted the rotation signal) and the PDC_SAP data from sector 5 to determine a rotation period of $15.776 \pm 0.005 \mathrm{~d}$. This is similar to the first reported period in the literature $(15.865 \pm 0.005 \mathrm{~d}$; Hensberge et al. 1981) and that reported by Balona et al. (2019), but is twice the period reported by Sikora et al. (2019c), who have detected the first harmonic of the true period. It is not ideal to mix the SAP and PDC_SAP data, but in this scenario such a procedure provides better results.

The star shows several pulsation modes: a triplet with a missing $+v_{\text {rot }}$ sidelobe, a closely spaced singlet (denoted by the dashed vertical line in Fig. 15), followed by two quintuplets. There appears to be further low amplitude modes between the multiplet groups, but at low $\mathrm{S} / \mathrm{N}$. The separation between the triplet and the singlet is $1.41 \mu \mathrm{Hz}$. The spacing between the two quintuplets is $\approx 19.7 \mu \mathrm{Hz}$ which is plausibly the large frequency separation for this star, indicating it is evolved.

\subsubsection{TIC 349945078}

TIC 349945078 (HD 57040) was classified as A2 EuCr by Renson \& Manfroid (2009). The TIC reports $T_{\text {eff }}=7203 \mathrm{~K}$, though literature
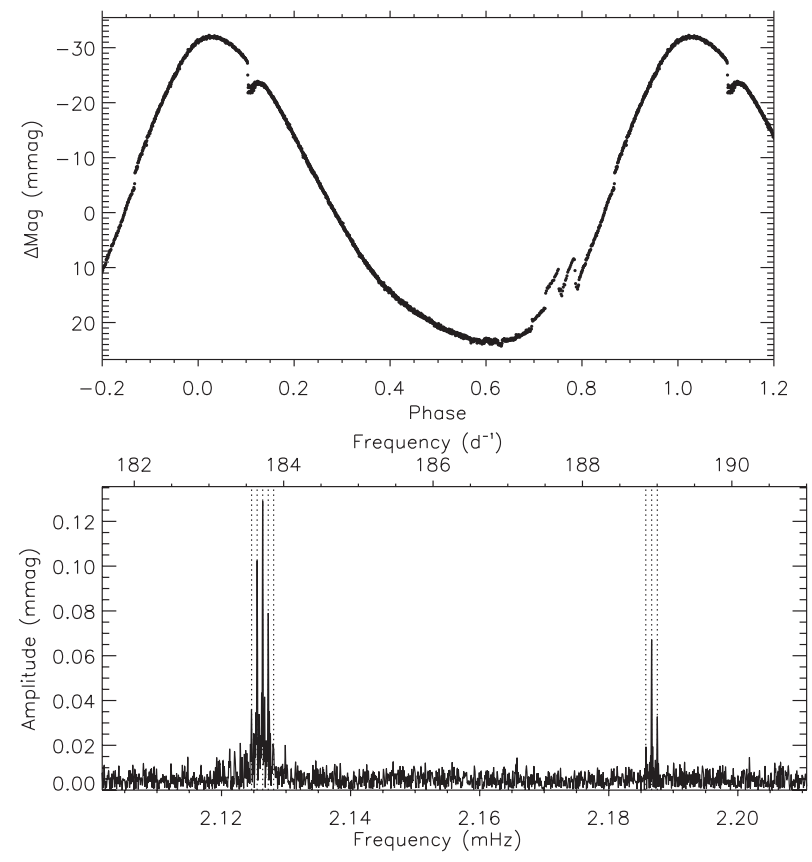

Figure 16. Top: phase folded SAP light curve of TIC 349945078, folded on a period of $13.4256 \mathrm{~d}$. The discontinuities, and the dip, are instrumental artefacts. Bottom: the pulsation modes in TIC 349945078. The vertical dotted lines show the components of obliquely split multiplets.

values for this parameter range from as low as $6518 \mathrm{~K}$ (Schofield et al. 2019) to as high as $8412 \mathrm{~K}$ (Ammons et al. 2006). Elkin et al. (2012) reported the presence of a $7.5 \mathrm{kG}$ magnetic field on the star. Martinez \& Kurtz (1994b) were unable to detect oscillations from ground-based observations, so it was not identified as an roAp star until Balona et al. (2019) used TESS data to detect two oscillation frequencies.

This star was observed in sectors 6-9, thus we revisit this star to provide a more detailed analysis of the variability with the availability of more data. To extract the rotation signal, we used the SAP data since the rotation signature in the PDC_SAP data has been significantly altered. This was evident upon visual inspection. We fitted a four element harmonic series to derive a rotation period of $13.4256 \pm 0.0008 \mathrm{~d}$ (Fig. 16). This is different from that presented by Balona et al. (2019) by a factor of two. We confirm our rotation period through the analysis of the oblique pulsations below.

For the pulsation analysis, we used the PDC_SAP data and pre-whitened the light curve to remove the rotation signal and instrumental artefacts. As a final correction, we removed outlying points. The resulting amplitude spectrum shows two groups of peaks composed of a quintuplet and a triplet (Fig. 16). The two modes in this star are separated by $\approx 60 \mu \mathrm{Hz}$ which is plausibly the large frequency separation.

\subsubsection{TIC 350146296}

TIC 350146296 (HD 63087) was classified as A7 IV by Houk \& Cowley (1975). However, due to the clearly spotted nature of this star, and the pulsation variability, we obtained several spectra with the SpUpNIC instrument, and find this star to be an F0p EuCr star (Fig. A12).

TIC 350146296 was first reported as an roAp star by Cunha et al. (2019), with Balona et al. (2019) providing an analysis of extra 

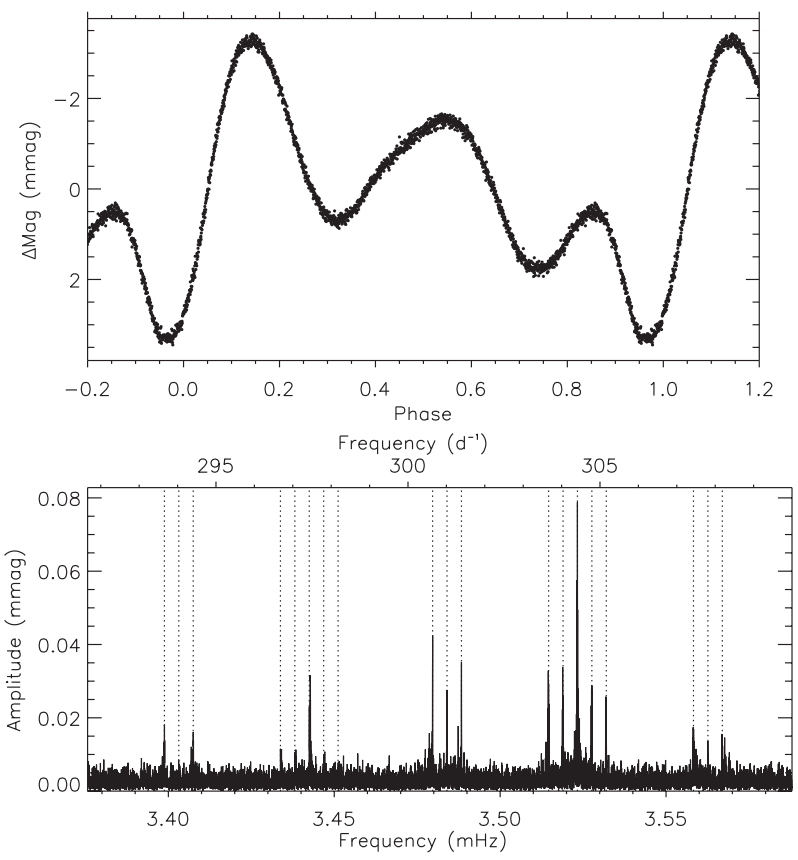

Figure 17. Top: phase folded light curve of TIC 350146296, folded on a period of $2.66387 \mathrm{~d}$. Bottom: the rich pattern of pulsation modes in TIC 350146296. The vertical dotted lines show the components of obliquely split multiplets.

sectors of data. There are now 13 sectors for this star from Cycle 1 which we analyse here. With the extended time base ( $357 \mathrm{~d})$ we derive a rotation period of $2.66387 \pm 0.00002 \mathrm{~d}$. The phased light curve, shown in Fig. 17, reveals a complex spot structure on this star.

There are five pulsation modes in TIC 350146296, all of which have been split into a multiplet by oblique pulsation. The multiplet structures allow us to conclude that the modes are alternating dipole and quadrupole modes, with three dipole modes and two quadrupole modes. All frequencies in this star show signs of variability, making precise frequency extraction difficult. All the modes are split by $\approx 40 \mu \mathrm{Hz}$ which, given the multiplet structures, we understand to be half the large frequency separation. Shi et al. (submitted) have conducted an extensive study on this star, to which we refer the reader for further details, although we provide the pulsation frequencies and rotation period in Table 1 here.

\subsubsection{TIC 431380369}

TIC 431380369 (HD 20880) was identified as a chemically peculiar star by Houk \& Cowley (1975) who gave the classification of $\mathrm{ApSr}(\mathrm{EuCr})$. It was later reported by Cunha et al. (2019) to be a new roAp star from the analysis of the TESS sector 2 data, after Joshi et al. (2016) presented a null result in the search for pulsations. The star was subsequently observed in sectors 6 and 13, where the significant gaps serve to produce a complicated window function. With the extended data, we are able to derive a more precise rotation period for this star of $5.19716 \pm 0.00005 \mathrm{~d}$.

This star shows a complex pulsation spectrum, as can be seen in Fig. 18, which is not helped by the significant gaps in the data. The mode at $0.805252 \pm 0.000002 \mathrm{mHz}\left(69.5737 \pm 0.0002 \mathrm{~d}^{-1}\right)$ is significantly broad, suggesting frequency and/or amplitude variability. The mode at $0.860412 \pm 0.000001 \mathrm{mHz}\left(74.3396 \pm 0.0001 \mathrm{~d}^{-1}\right)$ also shows amplitude variability, but includes a rotationally split

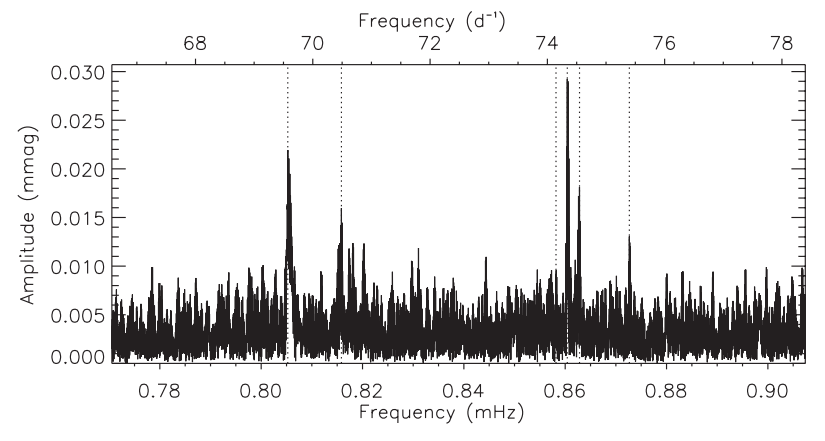

Figure 18. Pulsation modes in TIC 431380369. The window function gives fine structure to the peaks. However, there is also significant amplitude and frequency variability in this star, as can be seen in the mode at $0.860412 \mathrm{mHz}$. The vertical dotted lines denote the extracted modes, apart from the $v-v_{\text {rot }}$ sidelobe of the highest amplitude peak which is lost in the noise.

sidelobe at $v+v_{\text {rot }}$. The four significant modes are listed in Table 1 . We extract two extra modes, and the sidelobe, in addition to those reported by Cunha et al. (2019). This is likely a combination of the longer data set where the noise is slightly lower, and the mode lifetime of the pulsations seen in this star. A detailed study, on a rotation period-by-rotation period basis, of this star is required to extract the full information from the TESS data.

\subsection{Known roAp stars prior to TESS launch}

In this section, we present an analysis of the roAp stars which were known prior to the launch of the TESS mission. As with the new roAp star discoveries, we present only new information on stars which have already been discussed in the literature.

\subsubsection{TIC 6118924}

TIC 6118924 (HD 116114) was observed in sector 10. The star was classified as F0Vp SrCrEu by Abt, Brodzik \& Schaefer (1979) and was identified as an roAp star by Elkin et al. (2005a) who discovered variability in the $\mathrm{Eu}$ II lines with a period around $21 \mathrm{~min}(0.790 \mathrm{mHz}$; $\left.68.26 \mathrm{~d}^{-1}\right)$. A search for photometric variability after the spectroscopic detection by Lorenz, Handler \& Kurtz (2005) returned a null result at an amplitude level of about 0.5 mmag in Johnson $B$.

The rotation period of TIC 6118924 was determined to be $27.6 \mathrm{~d}$ by Landstreet \& Mathys (2000) which is longer than the TESS data span. We see no evidence of this period in the light curve. We detect one significant frequency at $0.76923 \pm 0.00004 \mathrm{mHz}$ $\left(66.462 \pm 0.003 \mathrm{~d}^{-1}\right)$ which is consistent with that found in the spectroscopic study (Fig. 19). This is the first photometric detection of the pulsation in this star.

\subsubsection{TIC 33601621}

TIC 33601621 (HD 42659) was classified as Ap SrCrEu by Houk \& Smith-Moore (1988). There are several occurrences of the stellar parameters in the literature with $T_{\text {eff }}$ ranging between 7450 and $7940 \mathrm{~K}$ (Kochukhov \& Bagnulo 2006; Balona et al. 2019). Kochukhov \& Bagnulo (2006) also provides $\log L / \mathrm{L}_{\odot}=1.48 \pm 0.09$ and $M=$ $2.1 \pm 0.1 \mathrm{M}_{\odot}$. There are also several measures of $\left\langle B_{z}\right\rangle$ which provide an average of $0.40 \pm 0.67 \mathrm{kG}$ (Hubrig et al. 2006; Kochukhov \& Bagnulo 2006; Bagnulo et al. 2015).

This star was discovered to be an roAp star by Martinez, Kurtz \& Ashley (1993b) with later observations providing more detailed 


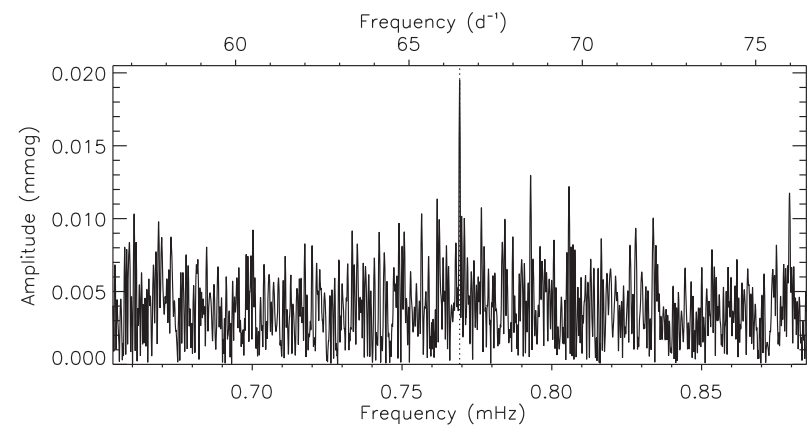

Figure 19. Amplitude spectrum of TIC 6118924 showing the single known pulsation mode.

results by Martinez \& Kurtz (1994a). The TESS sector 6 observations have been previously analysed by Balona et al. (2019), with a more detailed analysis presented by Holdsworth, Saio \& Kurtz (2019), analysing the dipole triplet. We do not present a re-analysis of the same data here, but refer the reader to the previous literature. It is worthy of note, however, that TIC 33601621 is the only roAp star known to be a member of a spectroscopic binary (SB1) system (Hartmann \& Hatzes 2015; Holdsworth et al. 2019).

\subsubsection{TIC 35905913}

TIC 35905913 (HD 132205) was classified as an Ap EuSrCr star by Houk \& Cowley (1975) and later discovered by Kochukhov et al. (2013) to be an roAp star through time-resolved spectroscopic analysis. Their data revealed a pulsation at $201.68 \pm 0.59 \mathrm{~d}^{-1}$ with an amplitude less than $100 \mathrm{~m} \mathrm{~s}^{-1}$. The star had previously been monitored photometrically for pulsations many times (Martinez \& Kurtz 1994b), but no signal was detected which is unsurprising given the small amplitude in the spectroscopic data.

TESS observed TIC 35905913 during sectors 11 and 12. The PDC_SAP light curves for these two sectors differ significantly. A clear rotation signature can be seen the sector 12 data, whereas the sector 11 data have a greatly reduced amplitude attributed to this variability. On inspection, the contamination factor for this star is high, at 0.28 , with the contaminating star about 18 arcsec away. According to Gaia DR2 Gaia Collaboration (2018), the Gband magnitude difference is 3.25 mag which suggests, under the assumption of negligible extinction differences, that the contaminant star is a mid G-type star.

By fitting a harmonic series of 5 components to the SAP sector 11 and PDC_SAP sector 12 data simultaneously, we derive a rotation period of $7.513 \pm 0.001 \mathrm{~d}$ (derived from $v_{1}$ in Fig. 20). Again, we reiterate that mixing the raw and pipeline corrected data is not ideal, but provides the best results in this case. After the removal of this harmonic series, there are clear signs of variability remaining in the data at low frequency ( $v_{2}$ and $v_{3}$ in the figure). The source of variability is uncertain given the proximity of the contaminating source. In fitting and removing a harmonic series to $v_{2}$, power remains around these frequencies in the amplitude spectrum, indicating an unstable frequency/amplitude which is common with star spots that change in size. This leads us to conclude that the contaminant is the source of the $v_{2}$ harmonic series. The origin of $v_{3}$ required further investigation, but could be a $g$ mode in the Ap or contaminant star.

We detect no pulsation in the roAp range to a limit of about $25 \mu \mathrm{mag}$ in the combined PDC_SAP flux data. This is unsurprising given the broad red bandpass of the TESS data, coupled with the low pulsation amplitude in spectroscopy.
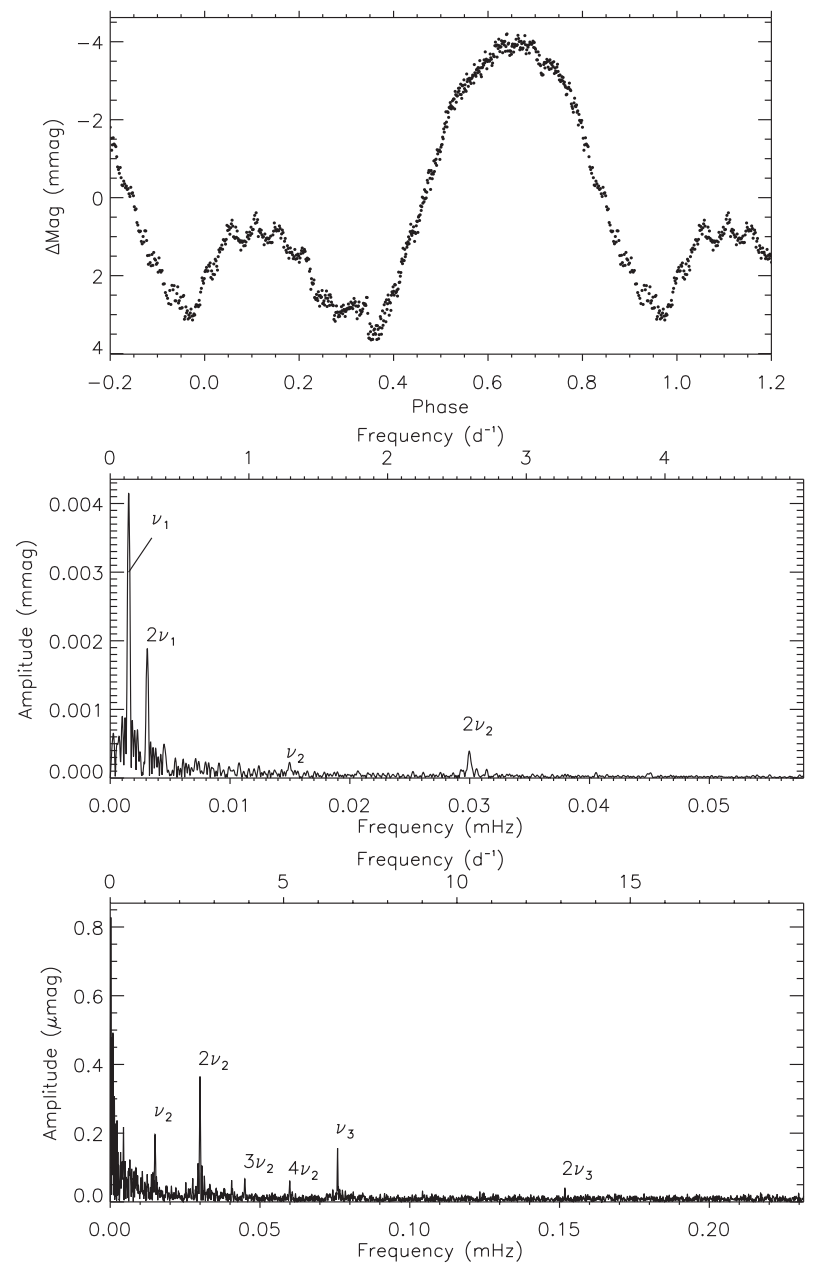

Figure 20. Top: rotation curve for TIC 35905913 . There is clear variability on a shorter time-scale. Middle: low-frequency amplitude spectrum showing the rotation frequency of the Ap star $\left(v_{1}\right)$ and its harmonic. $v_{2}$ and its harmonic are also indicated. Bottom: low-frequency amplitude spectrum after removing the Ap star rotation signature. Further harmonics of $\nu_{2}$ are evident, with an additional mode, $v_{3}$, and its harmonic labelled.

\subsubsection{TIC 44827786}

TIC 44827786 (HD 150562) was classified as F5Vp SrCrEu by Buscombe \& Foster (1995) and discovered to be an roAp star by Martinez \& Kurtz (1992) with a period of $10.75 \mathrm{~min}$.

The TIC gives $T_{\text {eff }}=7350 \mathrm{~K}$, which is consistent with that obtained using $u v b y \mathrm{H} \beta$ photometry from Joshi et al. (2016) and the Moon \& Dworetsky (1985) calibration. However, several literature sources give a significantly lower value: $6390 \mathrm{~K}$ (Gaia Collaboration 2018), $6620 \mathrm{~K}$ (McDonald et al. 2017), and 6820 K (Ammons et al. 2006). The star appears to be slowly rotating, with Elkin et al. (2008) obtaining a $v \sin i=1.5 \pm 0.5 \mathrm{~km} \mathrm{~s}^{-1}$ and Mathys et al. (2020b) finding no obvious signature of rotational variation from TESS data. This star shows variation of the mean magnetic field modulus from 4.7 to $5.0 \mathrm{kG}$ (Mathys 2017), with the mean longitudinal field changing between 1.2 and 1.7 kG (Bagnulo et al. 2015; Mathys 2017).

The mean magnetic field modulus was found to be $4.8 \mathrm{kG}$ (Kudryavtsev \& Romanyuk 2003), while more recently Bagnulo et al. (2015) obtained a value of $\sim 2 \mathrm{kG}$.

We detect two significant modes of pulsation in the TESS sector 12 data (Fig. 21). The highest amplitude mode at a frequency of 


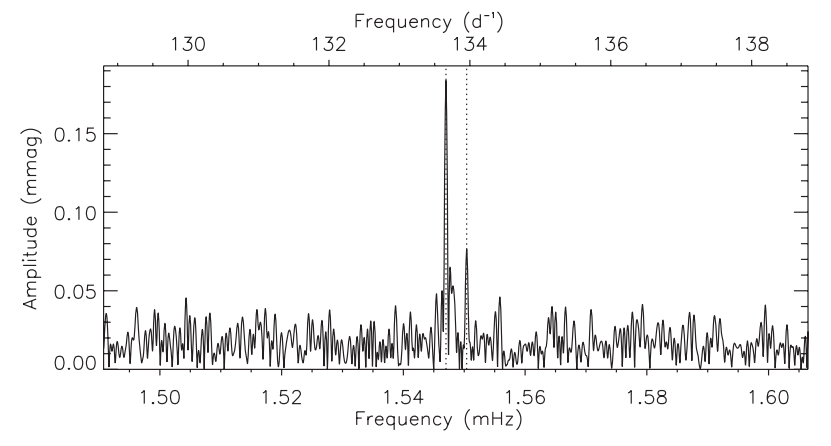

Figure 21. The pulsation signatures seen in TIC 44827786. This is the first detection of the highest amplitude mode, whilst the low amplitude mode is reported in the literature.

$1.54700 \pm 0.00002 \mathrm{mHz}\left(133.661 \pm 0.002 \mathrm{~d}^{-1}\right)$ is separated from the previously known pulsation mode $(1.55040 \pm 0.00005 \mathrm{mHz}$; $133.955 \pm 0.004 \mathrm{~d}^{-1}$ ) by $3.40 \pm 0.05 \mu \mathrm{Hz}$ which is plausibly the small frequency separation.

\subsubsection{TIC 49332521}

TIC 49332521 (HD 119027) is classified on SIMBAD as an $\alpha^{2} \mathrm{CVn}$ star, but Martinez et al. (1998a) found no rotational signal, and suggested a period of greater than 6 months if there is any rotational variation. Mathys et al. (2020b) also found no rotational variation. Its spectral type is $\mathrm{Ap} \mathrm{SrEu}(\mathrm{Cr})$ (Houk 1982). It has been known to be an roAp star for thirty years (Martinez \& Kurtz 1991b), and has a rich p-mode oscillation spectrum (Martinez, Koen \& Sullivan 1998b). Magnetic field properties have been measured for HD 119027 (Mathys 2017), with a measured value for $B_{z}$ of $0.5 \mathrm{kG}$, and multiple $\langle B\rangle$ measurements around $3.17 \mathrm{kG}$.

TESS observed HD 119027 in sector 11, with multiple oscillation frequencies discussed by Mathys et al. (2020b). Here we detect 7 independent modes in TIC 49332521. Six of these modes are almost the same as presented by Martinez et al. (1998b): their five roughly equally split modes (separated by $\approx 26 \mu \mathrm{Hz}$ ) plus their $v_{2 p}$ mode. Our additional mode is found at a lower frequency $\left(1.57070 \pm 0.00005 \mathrm{mHz} ; 135.709 \pm 0.004 \mathrm{~d}^{-1}\right)$ which is separated from the next mode by $264 \mu \mathrm{Hz}$. This means that modes spanning about five radial orders are not excited to a detectable amplitude in this star, which although is unusual, has been seen in other roAp stars, an excellent example of this being TIC 139191168 (HD 217522; Section 4.3.10).

We show in Fig. 22 the amplitude spectrum and the identified modes. Those marked by vertical dotted lines are separated by multiples of $\approx 26 \mu \mathrm{Hz}$ while those with dashed lines do not fit this pattern. We suspect, given the stellar parameters, that this separation is half of the large frequency separation, such that $\Delta v \approx 52 \mu \mathrm{Hz}$ for TIC 49332521. The separation between the two highest amplitude modes $(1.78 \mu \mathrm{Hz})$ could represent the small frequency separation in this star, although this value is different from the value of $1.95 \mu \mathrm{Hz}$ proposed by Martinez et al. (1998b).

\subsubsection{TIC 69855370}

TIC 69855370 (HD 213637) was observed in sector 2. The star is classified as A (pEuSrCr) by Houk \& Smith-Moore (1988) and was identified as an roAp star by Martinez et al. (1998c) who reported the discovery of two pulsation modes with periods around 11-12 min,

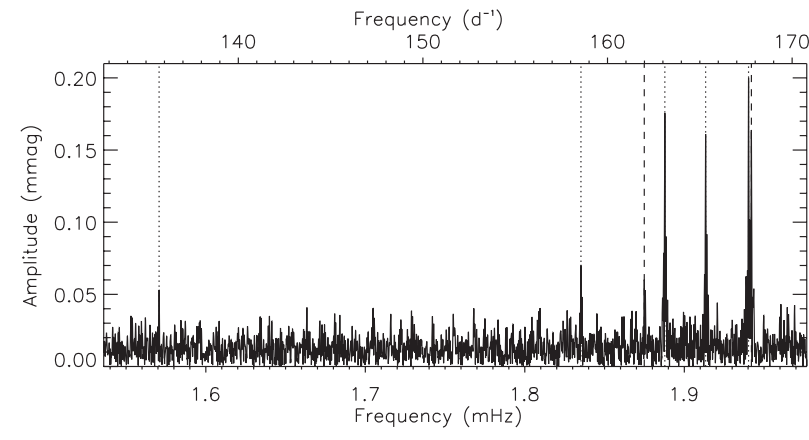

Figure 22. The pulsation signatures seen in TIC 49332521. Modes marked with dotted lines are separated by multiples of $\approx 26 \mu \mathrm{Hz}$ while those with dashed lines do not fit this pattern.

while acknowledging that the frequency values identified needed to be confirmed due to the presence of $1 \mathrm{~d}^{-1}$ aliases in the data. Based on the TESS data, Cunha et al. (2019) confirmed the presence of two pulsation modes in the same period range, the first corresponding to one of the modes identified by Martinez et al. (1998c) and the other differing from their second mode by $1 \mathrm{~d}^{-1}$.

No sign of rotational modulation was found by Cunha et al. (2019) in their analysis of the TESS data for this star. Nevertheless, based on the measurement of a projected rotational velocity of $v \sin i=$ $3.5 \pm 0.5 \mathrm{~km} \mathrm{~s}^{-1}$, Kochukhov (2003) suggested a rotation period of $25 \mathrm{~d}$. The lack of a rotational modulation in the TESS data could result from an unfavourable alignment ( $i$ or $\beta$ close to zero), as discussed by Cunha et al. (2019), or be a result of a long rotation period (Mathys et al. 2020b).

Since the TESS sector 2 data have been analysed in detail, we refer the reader to Cunha et al. (2019) for further discussion, but include the pulsation frequencies in Table 1 for completeness.

\subsubsection{TIC 93522454}

TIC 93522454 (HD 143487) was observed in sector 12. The star is classified as A3 SrEuCr in Renson \& Manfroid (2009). It was identified as an roAp star by Elkin et al. (2010), following the analysis of high time resolution spectra obtained with UVES from which a low amplitude pulsation with a period around $10 \mathrm{~min}$ was inferred. The roAp nature of this star was later confirmed by Kochukhov et al. (2013) based on the analysis of a larger set of UVES spectra which allowed the detection of the pulsations in the lines of rareearth elements as well as in the core of $\mathrm{H}_{\alpha}$ and the determination of a pulsation period of $9.63 \mathrm{~min}\left(1.730 \mathrm{mHz} ; 149.47 \mathrm{~d}^{-1}\right)$ with amplitudes up to $110 \mathrm{~m} \mathrm{~s}^{-1}$. No rotation period is reported in the literature for this star.

The TESS data do not show a clear pulsation signal. We show, in Fig. 23, the amplitude spectrum in the frequency region where pulsations have been previously found. We indicate the possible location of the pulsation in this star with an arrow, but caution its presence. The lack of an obvious detection is not surprising given the low amplitude radial velocity variations reported.

\subsubsection{TIC 125297016}

TIC 125297016 (HD 69013) was observed in sector 7. The star is classified as ApEuSr by Houk \& Smith-Moore (1988). It was identified as an roAp star with a pulsation period around $11 \mathrm{~min}$ by Elkin et al. (2011), following the analysis of high time resolution 


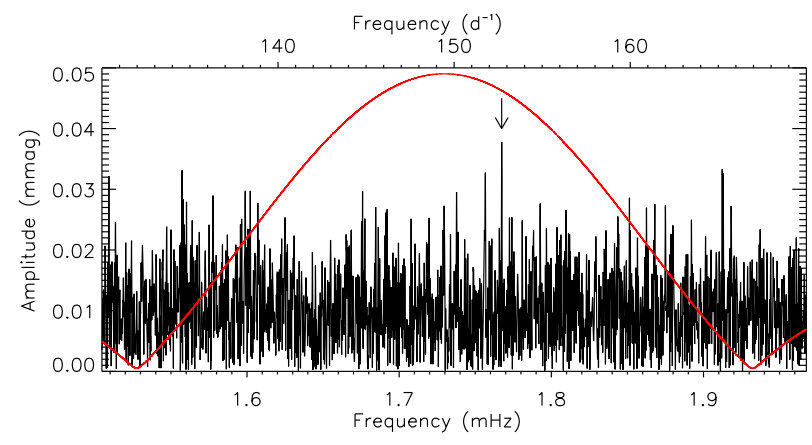

Figure 23. The amplitude spectrum of TIC 93522454 in the frequency region of interest. We see no clear indication of pulsation, but mark with an arrow a possible signature. The smooth solid red curve represents the shape of the peak used by Kochukhov et al. (2013) to provide a pulsation frequency of $1.73 \mathrm{mHz}\left(149.47 \mathrm{~d}^{-1}\right)$, demonstrating the broad frequency range where the mode may be found.

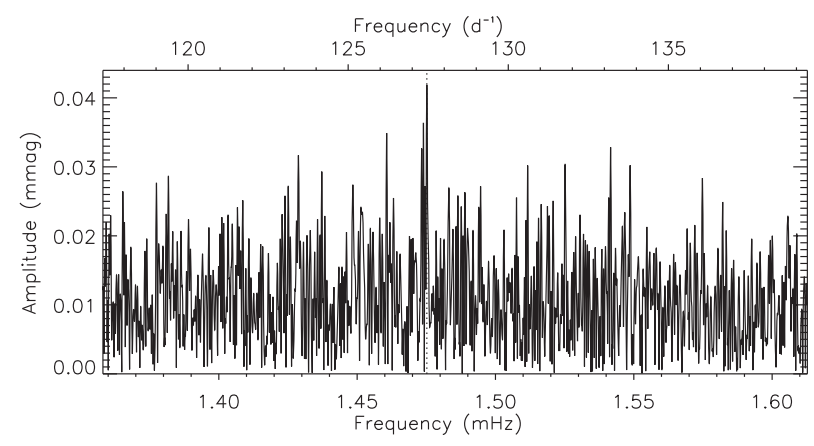

Figure 24. The amplitude spectrum of TIC 125297016 in the frequency region of interest. The highest amplitude peak is at a frequency of $1.47519 \mathrm{mHz}$ which is similar to that reported in the literature, however we are not confident in the detection.

spectra obtained with UVES. Pulsational variability with a similar period was also detected in photometric data published in the same work. The roAp nature of this star was confirmed by Kochukhov et al. (2013) based on the analysis of a larger set of UVES spectra which allowed the detection of the pulsations in the lines of rare-earth elements and the determination of a pulsation period of $11.22 \mathrm{~min}$ $\left(1.4854 \mathrm{mHz} ; 128.34 \mathrm{~d}^{-1}\right)$. No rotation period is reported in the literature and no sign of rotation variability was found by Mathys et al. (2020b) in their analysis of the TESS data for this star.

The TESS data of this star do not clearly show the known pulsation frequency. A significant peak is detected at a frequency of $1.47519 \pm 0.00005 \mathrm{mHz}\left(127.456 \pm 0.004 \mathrm{~d}^{-1}\right.$; Fig. 24) which is similar to the spectroscopic literature value, but further TESS observations are required to reduce the noise in the data to allow a confident photometric detection of the pulsation.

\subsubsection{TIC 136842396}

TIC 136842396 (HD 9289) is classified as A(p) SrEuCr by Houk \& Swift (1999b) and its mean magnetic field modulus was determined to be $\langle B\rangle=2.0 \mathrm{kG}$ (Ryabchikova et al. 2007). The star was discovered to be an roAp star by Kurtz, Martinez \& Tripe (1994b) who identified three pulsation modes in the amplitude spectrum of photometric data. Later, Gruberbauer et al. (2011) observed TIC 136842396 with the MOST satellite and found a greater number of frequencies, but with few in agreement with the first study. TESS observed TIC 136842396

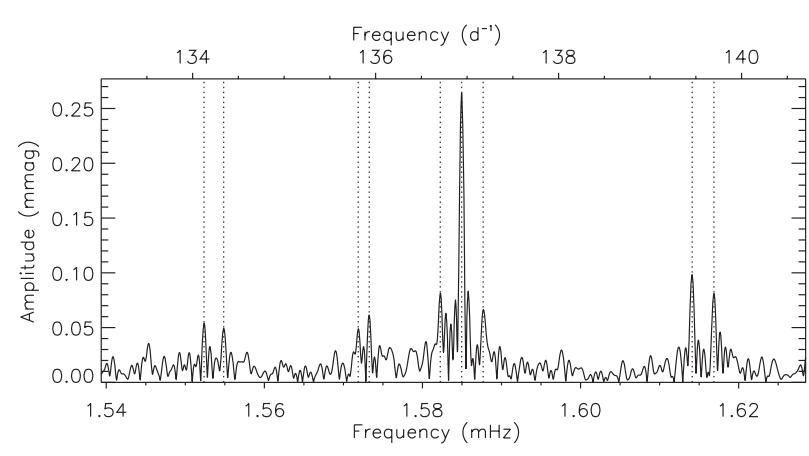

Figure 25. The pulsation signatures seen in TIC 136842396. The vertical dotted lines represent the extracted peaks, all of which are, in their groups, separated by integer multiples of the rotation frequency.

during sector 3, with Balona et al. (2019) presenting the first analysis of those data.

Here, we derive a rotation period of the star from the SAP data since the pipeline has clearly altered the astrophysical signal. We measure a rotation period of $8.660 \pm 0.006 \mathrm{~d}$, which is different from that provided by Balona et al. (2019). The pulsation spectrum for this star poses questions due to the presence, and lack thereof, of many rotationally split sidelobes. We extract nine significant peaks from the pre-whitened light curve of this star, as shown in Fig. 25. Given the lack of complete multiplets, we investigated the frequency separation between the assumed mode frequencies and found a reoccurrence of a separation of $\approx 31 \mu \mathrm{Hz}$. Under the assumption that the highest amplitude mode is a quadrupole mode with missing $\pm v_{\text {rot }}$ sidelobes, the pulsation spectrum consists of: a quadrupole mode showing only the $v_{\text {rot }}$ sidelobes, a dipole doublet split by the rotation frequency (with the pulsation mode being the highest amplitude peak), the quadrupole mode that is missing the $\pm v_{\text {rot }}$ sidelobes, and a final quadrupole mode showing only the $v_{\text {rot }}$ sidelobes. These tentative mode identifications imply a large frequency separation of $\approx 62 \mu \mathrm{Hz}$.

\subsubsection{TIC 139191168}

TIC 139191168 (HD 217522) was observed in sector 1. The star is classified as A5 SrEuCr in Renson \& Manfroid (2009) and has an upper limit on $\langle B\rangle$ of $1.5 \mathrm{kG}$ (Ryabchikova, Kochukhov \& Bagnulo 2008). It was first identified as an roAp star with pulsation periods around $13.72 \mathrm{~min}\left(1.2151 \mathrm{mHz} ; 104.98 \mathrm{~d}^{-1}\right)$ by Kurtz (1983), based on photometric data. The authors noted a significant night-to-night pulsation amplitude variability which was confirmed by a multisite photometric campaign conducted by Kreidl et al. (1991) in 1989. In addition to the pulsational variability around the period detected earlier, the latter data revealed a new pulsation with a period around $8.26 \mathrm{~min}\left(2.0174 \mathrm{mHz} ; 174.23 \mathrm{~d}^{-1}\right)$. In 2008, further photometric data, as well as high time resolution UVES/ESO spectra were collected. The analysis of these data, published by Medupe et al. (2015), showed that pulsational variability around both periods was still present.

The Cycle 1 TESS data for this star were analysed by Cunha et al. (2019) who detected four modes with frequencies in the interval $1.2008-1.2152 \mathrm{mHz}$, thus in the frequency region where all earlier data sets exhibited pulsations. However, the higher frequency mode, detected in the 1989 and 2008 data sets, was not detected in the TESS data. Similar to the earlier ground-based data, the TESS data showed evidence for amplitude and/or frequency modulation. No rotation period is reported in the literature for this star with Mathys 


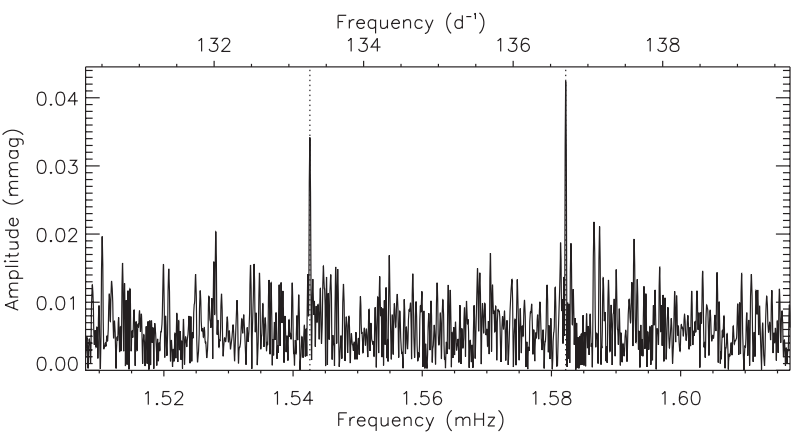

Figure 26. The pulsation signatures seen in TIC 146715928. The vertical dotted lines represent the extracted peaks, which are separated by $40 \mu \mathrm{Hz}$.

et al. (2020b) classifying the star as a super slowly rotating Ap (ssrAp) star, although the longitudinal field measurements reported in the catalogue by Bagnulo et al. (2015) do show some indication of variability on the time-scale of a few years. No sign of rotation variability was found in any of the published analyses of the TESS data (Cunha et al. 2019; David-Uraz et al. 2019; Mathys et al. 2020b).

Since the TESS data for this star have already been analysed in detail, we refer the reader to those works, but include the pulsation frequencies in Table 1 for completeness.

\subsubsection{TIC 146715928}

TIC 146715928 (HD 92499) was classified as A2p SrEuCr (Houk 1978 ) with $T_{\text {eff }}$ values ranging from $7200 \mathrm{~K}$ to $7810 \mathrm{~K}$ (Hubrig \& Nesvacil 2007; Freyhammer et al. 2008b; Elkin et al. 2010), and $\log g=4.1 \pm 0.2\left(\mathrm{~cm} \mathrm{~s}^{-2}\right)$. Elkin et al. (2010) also provided a full chemical abundance analysis of this star. A mean magnetic field modulus of $8.5 \mathrm{kG}$ was derived from FEROS spectra by Hubrig \& Nesvacil (2007) which was corroborated by Mathys (2017) who also suggested the rotation period of TIC 146715928 must be greater than $5 \mathrm{yr}$. TIC 146715928 has a $v \sin i \approx 3.5 \mathrm{~km} \mathrm{~s}^{-1}$ (Hubrig \& Nesvacil 2007; Elkin et al. 2010).

Rapid oscillations were found in the spectra of TIC 146715928 by Elkin et al. (2010) with a period of $10.4 \pm 0.3 \mathrm{~min}$. TESS observed the star during sectors 9 and 10. Mathys et al. (2020b) used the data to classify the star as an ssrAp star from the lack of rotation signal. They also provided a brief analysis of the two pulsation modes in this star that are separated by $40 \mu \mathrm{Hz}$, which could be the large frequency separation, or half of it. We present the extracted mode frequencies in Table 1, and show a plot of the amplitude spectrum in Fig. 26.

\subsubsection{TIC 167695608}

TIC 167695608 (TYC 8912-1407-1) was observed in 12 of the 13 sectors of Cycle 1 (all but sector 5). The star was classified as F0p SrEu(Cr) by Holdsworth et al. (2014a), who collected spectra to confirm its peculiar nature following the detection of rapid variability, with a period around $11 \mathrm{~min}\left(1.532 \mathrm{mHz} ; 132.38 \mathrm{~d}^{-1}\right)$ in a survey conducted using the SuperWASP archive (Pollacco et al. 2006).

The first two sectors of Cycle 1 TESS data were analysed by Cunha et al. (2019) who confirmed the pulsation detected previously and identified two more modes at slightly higher frequencies. A followup analysis of TESS data collected up to sector 7 revealed yet another pulsation mode at slightly lower frequency than the modes detected previously (Balona et al. 2019). Altogether, the TESS data up to sector 7 had revealed four pulsation frequencies. No sign of rotational

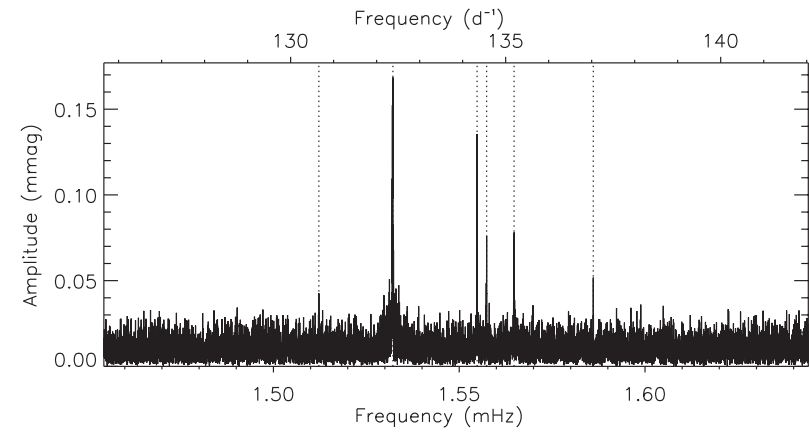

Figure 27. The pulsation signatures seen in TIC 167695608. There is significant frequency variability in most modes.

variability was found for this star in the SuperWASP (Holdsworth et al. 2014a) or in the year-long TESS Cycle 1 data (Mathys et al. 2020b).

With the longer data set, we are now able to detect six pulsation frequencies in this star (Fig. 27), most of which show significant frequency variability, evidenced by broad ragged peaks in the amplitude spectrum that are not cleanly extracted when pre-whitened. There is no clear pattern to the splitting of the pulsation modes, thus inhibiting the determination of the large or small frequency separation in this star.

\subsubsection{TIC 168383678}

TIC 168383678 (HD 96237) is an roAp star discovered by Elkin et al. (2011) and confirmed by Kochukhov et al. (2013) who determined the following stellar parameters: $T_{\text {eff }}=7800 \mathrm{~K}, \log g=4.3\left(\mathrm{~cm} \mathrm{~s}^{-2}\right)$, $v \sin i=6 \mathrm{~km} \mathrm{~s}^{-1}$ and a mean magnetic field modulus of $\langle B\rangle=2.9 \mathrm{kG}$. TIC 168383678 has a spectral classification of A4 SrEuCr (Renson $\&$ Manfroid 2009) and a rotation period of 20.91 d (Freyhammer et al. 2008b). The dominant pulsation mode was reported at $1.2 \mathrm{mHz}$ (Elkin et al. 2011; Kochukhov et al. 2013).

TESS observed the star during sector 9. The SPOC pipeline has removed the astrophysical signal associated with the rotation period in this star, so we use the SAP data. Upon inspection of the light curve, it is not clear if we have observed one rotation cycle or half of it. Inspection of the two observed light minima show subtly different shapes (Fig. 28). The data for this star span just $24.2 \mathrm{~d}$ which is just a little longer than the rotation period reported in the literature. From the light curve, we estimate a rotation period of either $\sim 21 \mathrm{~d}$ or $\sim 42 \mathrm{~d}$.

We detect a significant peak in the amplitude of the SAP light curve at a frequency of $1.19610 \pm 0.00003 \mathrm{mHz}\left(103.343 \pm 0.003 \mathrm{~d}^{-1}\right)$ which has an $\mathrm{S} / \mathrm{N}$ of 7.6. There are, perhaps, further modes in this star at slightly higher frequencies than that detected, but more data are required to confirm their presence.

\subsubsection{TIC 170419024}

TIC 170419024 (HD 151860) was classified as Ap SrEu(Cr) (Houk \& Cowley 1975). Kochukhov et al. (2013) measured a mean magnetic field modulus of $2.5 \mathrm{kG}$, and detected rapid pulsations through the analysis of high-resolution spectra collected with UVES, after the null detection in photometry (Martinez \& Kurtz 1994b). An average pulsation period of $12.30 \pm 0.09$ min was established from the radial velocity of lines of rare earth elements. Some lower amplitude 

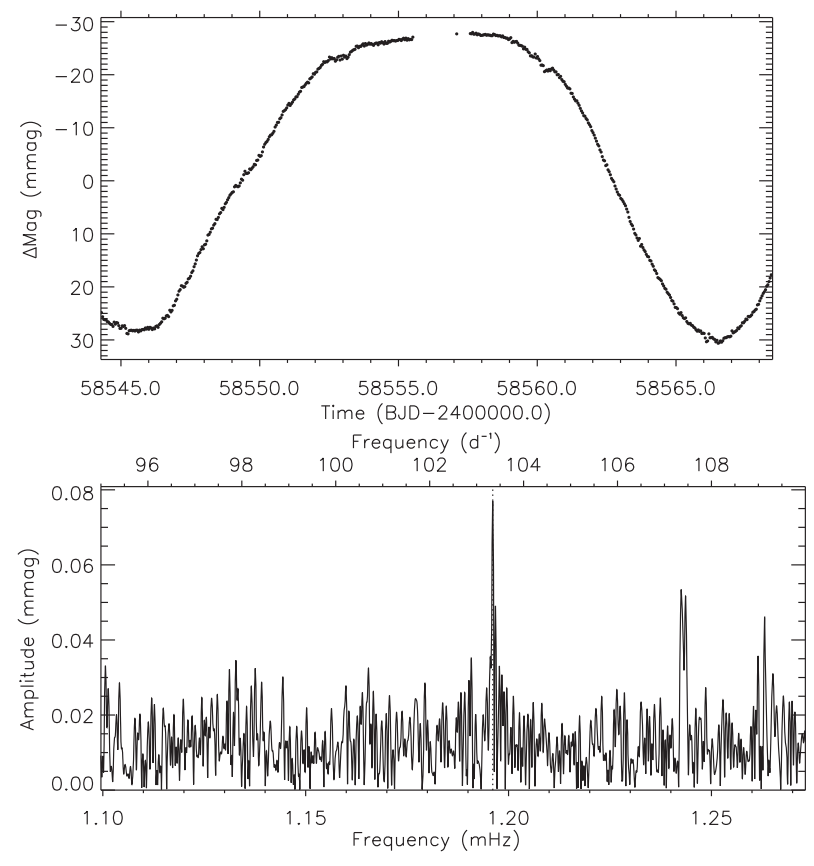

Figure 28. Top: the SAP light curve of TIC 168383678. It appears that one cycle is completed, but inspection of the unequal light minima suggests we may be seeing only half of a rotation cycle. Bottom: the amplitude spectrum of TIC 168383678 with the known pulsation mode indicated. There may be further modes at higher frequency, but with low significance in this data set.

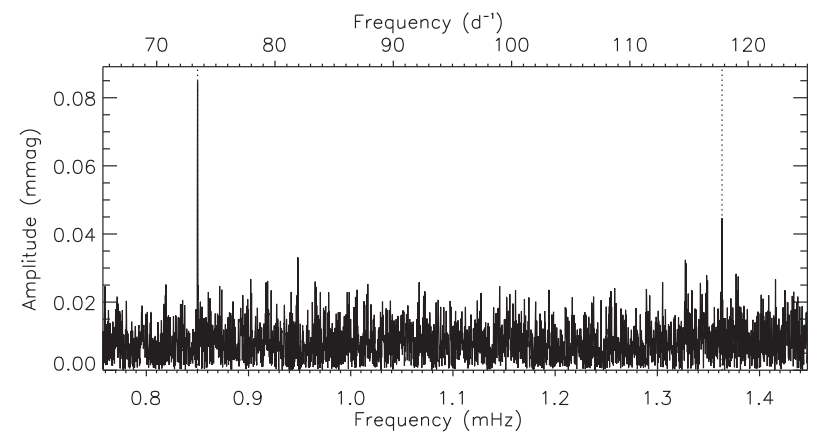

Figure 29. The pulsation signatures seen in TIC 170419024. The vertical dotted lines represent the extracted peaks.

peaks at other frequencies were detected indicating this star to be a multiperiodic roAp star.

The TESS sector 12 data show this star to be an ssrAp star (Mathys et al. 2020b) since the data show no rotation signature. Mathys et al. (2020b) also commented on the pulsation modes in this star, as detected in the TESS data. There is a significant peak at $0.85014 \pm 0.00002 \mathrm{mHz}\left(73.452 \pm 0.002 \mathrm{~d}^{-1}\right)$ which was not reported by Kochukhov et al. (2013), and a second peak at $1.36330 \pm 0.00004 \mathrm{mHz}\left(117.789 \pm 0.003 \mathrm{~d}^{-1}\right.$; Fig. 29) which is consistent with the spectroscopic analysis.

\subsubsection{TIC 173372645}

TIC 173372645 (HD 154708), with a rotation period of $5.37 \mathrm{~d}$, an effective temperature of $7200 \mathrm{~K}$ (Joshi et al. 2016), $\log g=$ $4.11\left(\mathrm{~cm} \mathrm{~s}^{-2}\right)$, and $v \sin i=4.0 \mathrm{~km} \mathrm{~s}^{-1}$, is classified as an Ap EuSrCr star (Renson \& Manfroid 2009). The dominant pulsation mode has

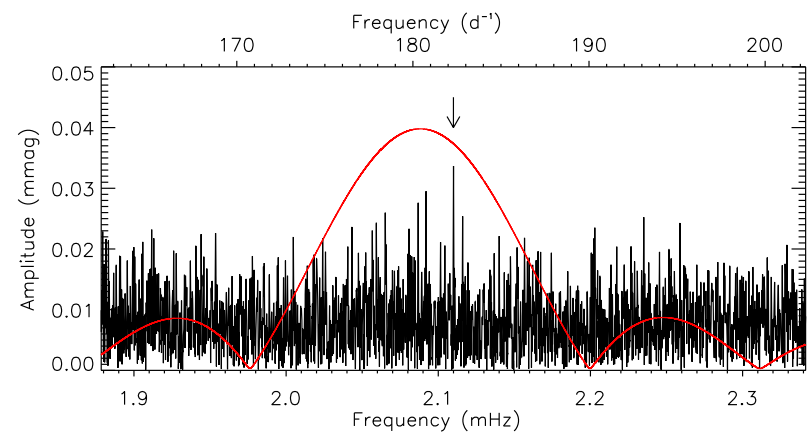

Figure 30. The amplitude spectrum of TIC 173372645. The arrow indicates a potential peak, whereas the broad smooth red curve represents the shape of the peak used by Kurtz et al. (2006b) to identify the pulsation mode in this star.

a frequency of $2.088 \mathrm{mHz}\left(180.4 \mathrm{~d}^{-1}\right)$, which was discovered by Kurtz et al. (2006b) through the analysis of high time resolution UVES spectra at an amplitude of just $\approx 60 \mathrm{~m} \mathrm{~s}^{-1}$. TIC 173372645 has a magnetic field of $24.5 \mathrm{kG}$, the strongest ever measured for an roAp star (Hubrig et al. 2005), and one of the strongest for an Ap star.

TESS observed TIC 173372645 in sector 12. The light curve is rotationally modulated with a period of $5.363 \pm 0.001 \mathrm{~d}$. There is no clear indication of pulsation in this star in the TESS data, only a few peaks in the amplitude spectrum in the frequency region where the pulsation was previously found (Fig. 30; Kurtz et al. 2006b). The low amplitude in the spectroscopic data seems to have translated to an undetectable peak in the TESS photometry.

\subsubsection{TIC 189996908}

TIC 189996908 (HD 75445) was classified as an $\mathrm{Ap} \mathrm{SrEu}(\mathrm{Cr}$ ) star by Houk (1982) and has a magnetic field strength of $\langle B\rangle=$ $2.99 \pm 0.04 \mathrm{kG}$ (Mathys et al. 1997). The stellar parameters were measured to be $T_{\text {eff }}=7700 \mathrm{~K}, \log g=4.3\left(\mathrm{~cm} \mathrm{~s}^{-2}\right)$ (Ryabchikova et al. 2004), $v \sin i \leq 2 \mathrm{~km} \mathrm{~s}^{-1}$ (Kochukhov et al. 2009a) and the Hipparcos parallax resulted in $\log L=1.17 \pm 0.06 \mathrm{~L}_{\odot}$ (Kochukhov \& Bagnulo 2006). Based on radial velocity measurements, Kochukhov et al. (2009a) discovered rapid oscillations in this star, with a main pulsation frequency at $1.85 \mathrm{mHz}\left(159.8 \mathrm{~d}^{-1}\right)$.

TESS observed TIC 189996908 in sectors 8 and 9. The data show no rotational modulation which implies a long rotation period, or an unfavourable view. The magnetic field measurements presented by Mathys (2017) show changes over short time periods, implying a low inclination angle, rather than slow rotation. The pulsation signal is weak in this star, and would perhaps be classed as noise if it was not previously known. We find a peak in the amplitude spectrum at $1.84163 \pm 0.00002 \mathrm{mHz}\left(159.117 \pm 0.002 \mathrm{~d}^{-1}\right)$ with an $\mathrm{S} / \mathrm{N}$ of 5.3 (Fig. 31). This is another example of how low amplitude modes in spectroscopic observations are difficult to observe photometrically.

\subsubsection{TIC 211404370}

TIC 211404370 (HD 203932) is classified as A5 SrEu in the catalogue of Renson \& Manfroid (2009). Gelbmann et al. (1997) derived $T_{\text {eff }}=7540 \pm 100 \mathrm{~K}$ and $\log g=4.1\left(\mathrm{~cm} \mathrm{~s}^{-2}\right)$ for this star, while the average magnetic field, $\langle B\rangle$, was estimated to be below $1 \mathrm{kG}$ (Ryabchikova et al. 2008), while the root mean square longitudinal field was reported to be $0.25 \pm 0.15 \mathrm{kG}$ (Bychkov, Bychkova \& 


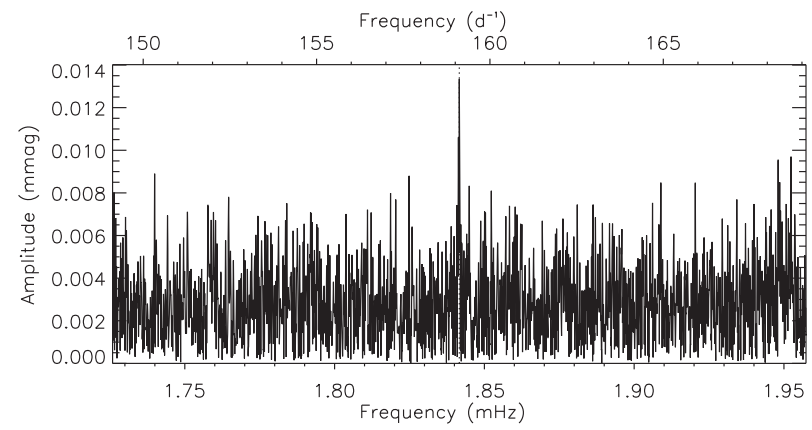

Figure 31. The amplitude spectrum of TIC 189996908 . The low S/N peak detection agrees with that found in spectroscopic observations.

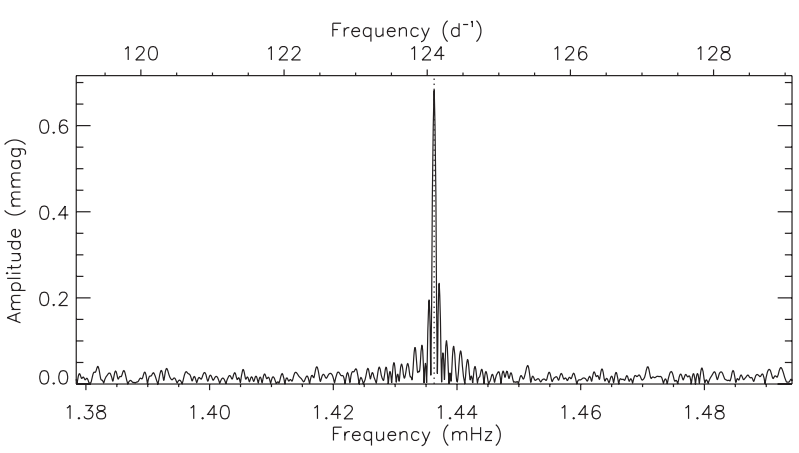

Figure 32. The single pulsation mode seen in TIC 268751602.

Madej 2009) and the $v \sin i$ was measured to be $4.7 \mathrm{~km} \mathrm{~s}^{-1}$ (Smalley et al. 2015). Pulsations were first detected in TIC 211404370 by Kurtz (1984) and it was later shown to have 4 significant peaks in the amplitude spectrum between 1.280 and $2.737 \mathrm{mHz}$ (Martinez, Kurtz \& Heller 1990).

This star was observed in TESS's sector 1, with the data analysed in Cunha et al. (2019). We find no significant difference in our analysis and that previously presented, so we refer the reader to that work. We include the rotation frequency, and the frequency of the two pulsation modes, in Table 1.

\subsubsection{TIC 237336864}

TIC 237336864 (HD 218495), with a spectral type of ApEuSr (Renson \& Manfroid 2009), was shown to have a $v \sin i$ of $16 \mathrm{~km} \mathrm{~s}^{-1}$ (Smalley et al. 2015) with a mean longitudinal magnetic field, $B_{z}$, of about $-1 \mathrm{kG}$ (Bagnulo et al. 2015). Cunha et al. (2019) derived $T_{\text {eff }}=7950 \pm 160 \mathrm{~K}$ and $\log g=4.1\left(\mathrm{~cm} \mathrm{~s}^{-2}\right)$. Rapid oscillations were first detected by Martinez \& Kurtz (1990) in this star.

This star was observed in sector 1, with the data analysed in Cunha et al. (2019) and by Kobzar (2020). The data show a complex pulsation pattern with, increasing frequency, a singlet, a doublet which is split by twice the rotation frequency, a quintuplet with components split by the rotation frequency, and a triplet of frequencies with each component split by twice the rotation frequency. Given the variety of different multiplet structures, a simple understanding of this star is not possible, but to develop a complete understanding is beyond the scope of this work. We provide the inferred pulsation frequencies and amplitudes in Table 1.

\subsubsection{TIC 268751602}

Houk \& Smith-Moore (1988) classified TIC 268751602 (HD 12932) as Ap SrEuCr, with Kochukhov et al. (2007) providing parameters of $T_{\text {eff }}=7620 \mathrm{~K}, \log g=4.15\left(\mathrm{~cm} \mathrm{~s}^{-2}\right)$, and $v \sin i=3.5 \mathrm{~km} \mathrm{~s}^{-1}$, and Ryabchikova et al. (2007) deriving $\langle B\rangle=1.7 \mathrm{kG}$.

Schneider \& Weiss (1990) and Kreidl (1990) independently collected high time resolution photometric data of TIC 268751602 and discovered variability with a period of about $11.6 \mathrm{~min}(1.4368 \mathrm{mHz}$; $\left.124.14 \mathrm{~d}^{-1}\right)$, thus announcing TIC 268751602 as an roAp star. This period was later refined by Schneider, Kreidl \& Weiss (1992) to be $11.7 \mathrm{~min}$, with the authors suggesting a rotation period of $3.5295 \mathrm{~d}$. Analysis of photometric data by Martinez, Kurtz \& van Wyk (1994) showed that TIC 268751602 was a single-mode pulsator with a period of $11.6 \mathrm{~min}$. The earlier identification of the rotation period was concluded to be likely incorrect. Kochukhov et al. (2007) identified variability in spectral lines of some elements in the atmosphere of TIC 268751602.

TESS observed this star during sector 3, with an analysis of those data presented by Balona et al. (2019). We confirm the lack of detection of rotation in this star, and agree with the pulsation frequency presented in that work, namely $1.436302 \pm 0.000006 \mathrm{mHz}$ $\left(124.0965 \pm 0.0005 \mathrm{~d}^{-1}\right)$. There is only one pulsation mode seen in these data of this star (Fig. 32).

\subsubsection{TIC 279485093}

TIC 279485093 (HD 24712, HR 1217) was classified as A9Vp SrEuCr by Abt \& Morrell (1995). Perraut et al. (2016) derived a temperature from bolometric flux and optical interferometry of $7235 \pm 280 \mathrm{~K}$, while Sikora et al. (2019a) derived $7150 \pm 170 \mathrm{~K}$ from spectroscopy. The mean magnetic field modulus for TIC 279485093 was determined to be $2.3 \mathrm{kG}$ (Ryabchikova et al. 2008). Detailed topology of the magnetic field of this star was reconstructed by Lüftinger et al. (2010) and Rusomarov et al. (2015) - the only roAp for which such constraint is available. Kurtz \& Marang (1987b) obtained a rotation period from the mean light variation of 12.45733 d, while Rusomarov et al. (2013) derived $12.45812 \mathrm{~d}$ from the longitudinal field measurements spanning $40 \mathrm{yr}$ and Kobzar et al. (2020) found a signal in the Fourier spectrum of the TESS data that may correspond to a rotation period of $12.44 \pm 0.02 \mathrm{~d}$. Kurtz (1981) first discovered $6.15 \mathrm{~min}\left(2.71 \mathrm{mHz} ; 234.1 \mathrm{~d}^{-1}\right)$ oscillations in this star, and Balona et al. (2019) list 10 independent frequencies. Many of these modes show the expected rotation-modulated amplitude variations, but unexplained variations in this modulation also occur on shorter time-scales of days (White et al. 2011). This star is one of the first discovered and best-studied roAp stars, appearing extensively in the literature.

As mentioned, TESS observed this star in sector 5. From the SAP data, we derive a rotation period of $12.578 \pm 0.008 \mathrm{~d}$ which is different from previous literature values (Fig. 33). The sector 5 SAP data, after removing points obviously affected by instrumental artefacts, span $25.63 \mathrm{~d}-\mathrm{a}$ little over twice the derived rotation period, thus this value should be treated with caution.

To inspect the pulsations in this star, we used the PDC_SAP light curve. The pulsation spectrum is known to be rich in this star, as evidenced by the middle panel in Fig. 33. We are able to extract 9 pulsation modes (as listed in Table 1) and 15 sidelobes that are split from the pulsation frequency by oblique pulsation. In comparison to the best ground-based photometric data set for this star (Kurtz et al. 2005), we detect all but their $v_{6}$ frequency $\left(2.789 \mathrm{mHz} ; 240.97 \mathrm{~d}^{-1}\right)$ although we see a power excess in the bottom panel of Fig. 33 around 

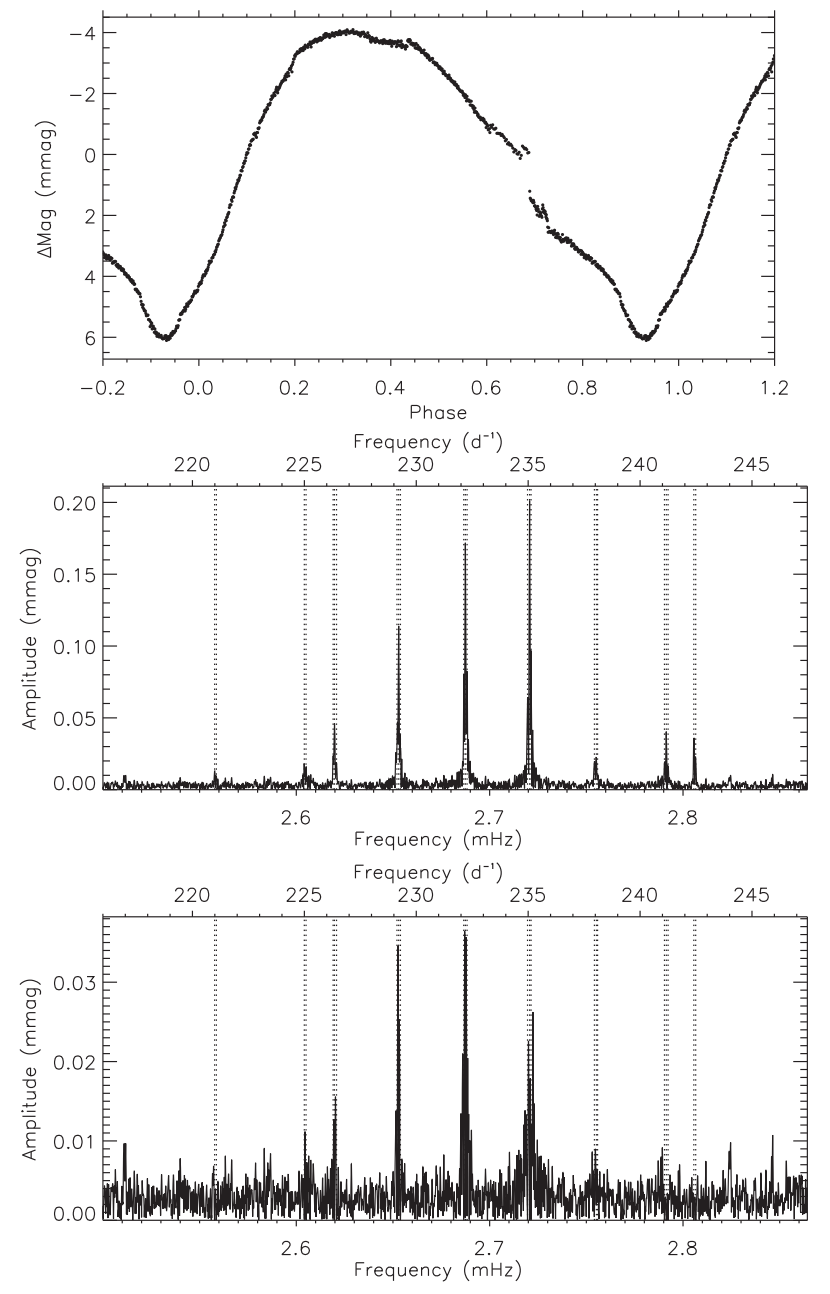

Figure 33. Top: the phase folded SAP light curve of TIC 279485093 , folded on a period of $12.578 \mathrm{~d}$. Middle: the amplitude spectrum of the PDC_SAP data. The vertical dotted lines represent the frequencies extracted. Bottom: amplitude spectrum of the residuals after removing 24 frequencies (9 pulsation modes and significant sidelobes). There is power remaining around the dominant modes which suggests significant frequency variability in this star. It seems likely there are further modes close to the noise level, too.

this frequency. We detect two further modes (our lowest frequencies) which likely correspond to two modes detected by Mkrtichian \& Hatzes (2005) via high-resolution spectroscopy.

\subsubsection{TIC 280198016}

TIC 280198016 (HD 83368, HR 3831) is a close visual binary, with the roAp star having spectral type A8V SrCrEu and the companion having type F9V (Corbally 1984). Sikora et al. (2019a) derive $T_{\text {eff }}=$ $7660 \pm 170 \mathrm{~K}$ which is consistent with the TIC value. TIC 280198016 shows a polarity reversing magnetic field strength that varies about zero mean with amplitude $0.74 \pm 0.07 \mathrm{kG}$ (Mathys 1991), and has a mean field modulus of $2.1 \mathrm{kG}$ (Kochukhov et al. 2004). The rotation period of TIC 280198016 was precisely determined to be $2.851976 \pm 0.000003 \mathrm{~d}$ (Kurtz et al. 1997a), with the star showing a single dipole pulsation mode at $123.38 \mathrm{~d}^{-1}$ that was exactly split into a septuplet by pulsation amplitude modulation with the rotation frequency. Baldry, Kurtz \& Bedding (1998b) found that the radial velocity variations were amplitude and phase

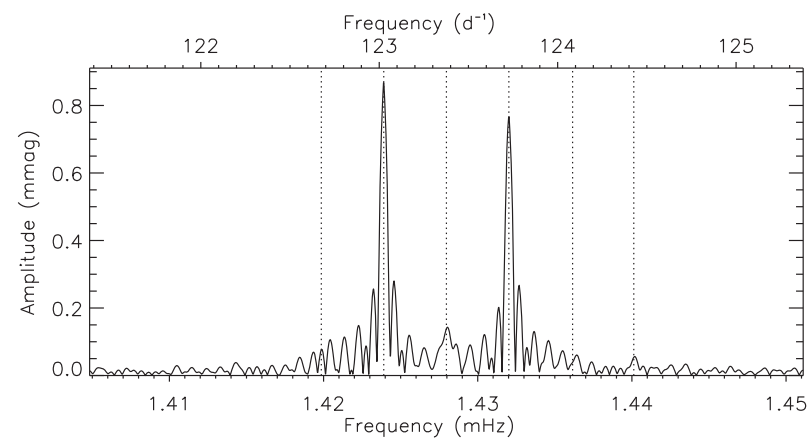

Figure 34. Amplitude spectrum of PDC_SAP light curve of TIC 280198016. We detect 6 of the 7 septuplet components found by Kurtz et al. (1997a).

modulated in the same manner as the photometric variations, first confirming the oblique pulsator model and ruling out the spotted pulsator model for roAp stars (Mathys 1985). A detailed study of pulsational line profile variations based on high-resolution spectroscopic observations was carried out by Kochukhov (2006). The surface distribution of the pulsation amplitude and phase was mapped by Kochukhov (2004) with the help of the Doppler imaging method - the only such analysis available for an roAp star.

The TESS sector 9 data of TIC 280198016 allow us to derive a rotation frequency (Table 1) which agrees with that of Kurtz et al. (1997a). We detect six significant peaks (Fig. 34) of the reported septuplet in TIC 280198016 and find that the mode shows frequency variability, as reported by Kurtz et al. (1997a). We also detect the second harmonic of the pulsation mode which forms a triplet centred on twice the pulsation frequency, although Kurtz et al. (1997a) show four harmonics.

\subsubsection{TIC 315098995}

TIC 315098995 (HD 84041) was classified as Ap SrEuCr by Houk (1982), and has effective temperature measurements of $8100 \mathrm{~K}$ (Mathys, Kharchenko \& Hubrig 1996), $7800 \mathrm{~K}$ and $7500 \mathrm{~K}$ from Elkin et al. (2008) obtained through the photometric calibrations of Moon \& Dworetsky (1985) and fitting synthetic spectra to the $\mathrm{H}_{\alpha}$ profile. TIC 315098995 is a magnetic star with Kochukhov \& Bagnulo (2006) reporting the first detection of a longitudinal magnetic field of $0.50 \pm 0.09 \mathrm{kG}$. The latest evaluation given in the FORS1 catalogue of stellar magnetic field measurements was $0.64 \pm 0.05 \mathrm{kG}$ measured from Balmer and metal lines (Bagnulo et al. 2015).

Martinez (1991) first reported the star as an roAp star with a pulsation period of about $14.6 \mathrm{~min}\left(1.14 \mathrm{mHz} ; 98.6 \mathrm{~d}^{-1}\right)$. Later Martinez et al. (1993a) clarified that the star pulsated in at least three modes with periods near $15 \mathrm{~min}$ and with frequency separations of about $30 \mu \mathrm{Hz}$. However, it was noted that these frequencies were not a complete description of the pulsation behaviour of TIC 315098995 . It exhibited mean light variations of a double-wave nature, indicating that both magnetic poles were visible. The rotation period determined from these observations was $3.69 \pm 0.01 \mathrm{~d}$. Elkin et al. (2008) reported maximum pulsation amplitude of $0.5 \mathrm{~km} \mathrm{~s}^{-1}$ and a pulsation amplitude from the $\mathrm{H}_{\alpha}$ core of $0.20 \mathrm{~km} \mathrm{~s}^{-1}$.

TESS observed TIC 315098995 during sectors 8 and 9. We cautiously combined the PDC_SAP data from sector 8 and the SAP sector 9 data to determine the rotation period since the SPOC pipeline has distorted the astrophysical signal in the latter. We measure a rotation 


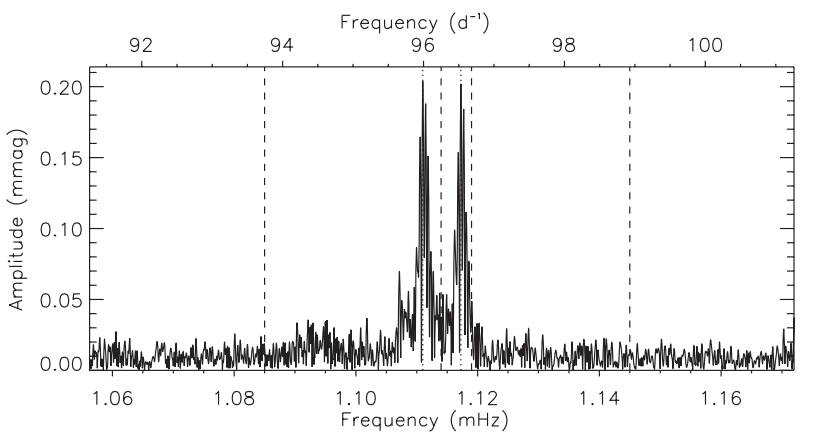

Figure 35. Amplitude spectrum of the two-sector light curve of TIC 315098995. There are at least two very variable peaks in this star. The vertical dashed lines represent the positions of the frequencies found by Martinez et al. (1993a).

period of $3.6884 \pm 0.0004 \mathrm{~d}$, which is consistent with the literature value.

The TESS data show this star to have a very variable pulsation signal, as reported by previous studies. We find two peaks (Fig. 35) that are split by twice the rotation frequency. We assume then, that we are seeing two sidelobes of a dipole triplet where the peak corresponding to the actual pulsation mode frequency is missing, implying that either $i$ or $\beta$ are close to $90^{\circ}$. This is corroborated when considering the results of Martinez et al. (1993a). We do not detect, in the combined light curve, the other modes reported to be present in this star. A careful inspection of the data, in shorter segments, is required to extract the full information on this star.

\subsubsection{TIC 322732889}

TIC 322732889 (HD 99563) was classified as A9 III star by Houk (1978) and with a Sr peculiarity added later (Bidelman 1981), although the star appears in the catalogue of Renson \& Manfroid (2009) as an F0 Sr. From high-resolution spectra, Elkin et al. (2008) found $T_{\text {eff }}=7700 \mathrm{~K}$ and $\log g=4.2\left(\mathrm{~cm} \mathrm{~s}^{-2}\right)$. The first measure of the longitudinal magnetic field gave $0.69 \pm 0.15 \mathrm{kG}$ (Hubrig et al. 2004), with two further measurements at different phases of $-0.24 \pm 0.07 \mathrm{kG}$ and $0.67 \pm 0.04 \mathrm{kG}$ (Hubrig et al. 2006). These detections were confirmed by Kochukhov \& Bagnulo (2006) who measured a field strength of $-0.392 \mathrm{kG}$.

Dorokhova \& Dorokhov (1998) first announced the detection of pulsation in TIC 322732889 with a period of about $11.17 \mathrm{~min}$ $\left(1.4921 \mathrm{mHz} ; 128.9 \mathrm{~d}^{-1}\right)$. Later, Handler \& Paunzen (1999) confirmed the roAp nature of the star with a period of $10.7 \mathrm{~min}$. Elkin, Kurtz \& Mathys (2005b) discovered remarkably large amplitude pulsations in the star for some spectral lines with amplitudes up to $5 \mathrm{~km} \mathrm{~s}^{-1}$ making this star the largest radial velocity amplitude roAp star known. A more detailed study by Handler et al. (2006) using multisite photometry found a single rotationally modulated mode with a frequency $1.558 \mathrm{mHz}\left(134.6 \mathrm{~d}^{-1}\right)$ connected with the rotation determined to be $2.912 \mathrm{~d}$. Further spectroscopic observations found little variation in the pulsation amplitude in TIC 322732889 (Kurtz, Elkin \& Mathys 2006a), while Freyhammer et al. (2009) claimed the presence of $\mathrm{H} \alpha$ spots caused by the settling of $\mathrm{He}$ in the presence of the magnetic field. Based on MOST observations, Gruberbauer et al. (2011) found a new candidate frequency independent of the primary multiplet.

TESS observed TIC 322732889 during sector 9. With those data, we derive a rotation period of $2.9114 \pm 0.0002 \mathrm{~d}$ which is consistent with that in the literature. As reported in previous works, we find a

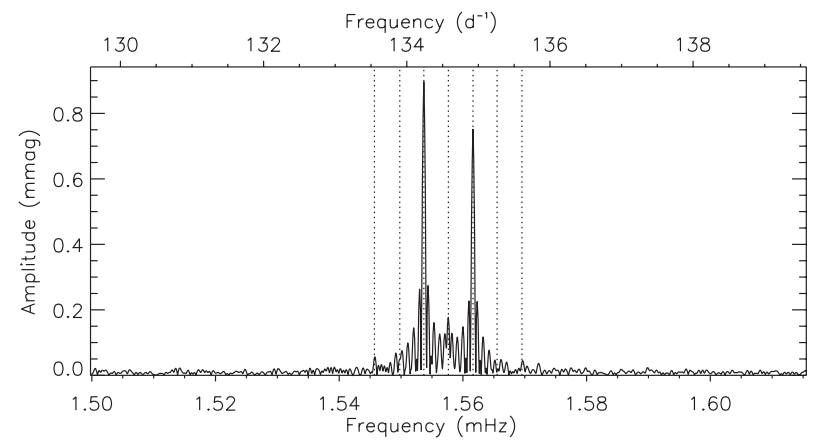

Figure 36. Amplitude spectrum of the light curve of TIC 322732889. The vertical dotted lines represent the septuplet components split by oblique pulsation.

septuplet of peaks in the amplitude spectrum separated by the rotation frequency. The central peak, representing the pulsation mode, is at $1.557657 \pm 0.000002 \mathrm{mHz}\left(134.5816 \pm 0.0002 \mathrm{~d}^{-1}\right.$; Fig. 36), and has a smaller amplitude than the first rotational sidelobes. We detect none of the candidate modes of Gruberbauer et al. (2011).

\subsubsection{TIC 326185137}

TIC 326185137 (HD 6532) was classified as Ap SrCrEu by Houk (1982), although there are a variety of different classifications (Skiff 2014). Effective temperature measurements for this star range from $7995 \mathrm{~K}$ by Bai et al. (2019) to $8382 \mathrm{~K}$ by McDonald et al. (2017). The $\log g$ value for this star is also poorly constrained in the literature, with an average value of $4.25\left(\mathrm{~cm} \mathrm{~s}^{-2}\right)$. Several measurements of the magnetic field have been made, with the $\langle B\rangle_{\mathrm{rms}}$ being $0.411 \mathrm{kG}$ (Mathys \& Hubrig 1997; Bychkov et al. 2009; Bagnulo et al. 2015).

TIC 326185137 was discovered to be an roAp star by Kurtz \& Kreidl (1985) with a period of $6.9 \mathrm{~min}\left(2.4155 \mathrm{mHz} ; 208.7 \mathrm{~d}^{-1}\right)$. They found a frequency triplet split by the rotation frequency of the star. Subsequently, the star was the subject of many ground-based photometric campaigns, with Kurtz et al. (1996b) concluding the star pulsates with a distorted dipole mode. The rotation period of this star has been refined many times in the literature, with an average period of 1.94 d (Kurtz \& Marang 1987a; Kurtz et al. 1996a; Renson \& Manfroid 2009; Balona et al. 2019).

TESS observed this star in sector 3, with an initial analysis of the data being presented by Balona et al. (2019) where it was noted that the multiplet structure was vastly different from previous $B$ photometric data. Kurtz \& Holdsworth (2020) explored this further, postulating two scenarios that could explain the striking difference between the two data sets: either the star has changed its pulsation axis or, more likely, we are seeing a depth dependence on the mode geometry revealed by the two wavelengths of observation.

From the data, we determine a rotation period of $1.9447 \pm 0.0001 \mathrm{~d}$, and a pulsation frequency of $2.402163 \pm 0.000007 \mathrm{mHz}\left(207.5469 \pm 0.0006 \mathrm{~d}^{-1}\right)$. We identify 4 of the 5 peaks of the distorted dipole multiplet (Fig. 37) in the TESS Cycle 1 data.

\subsubsection{TIC 340006157}

TIC 340006157 (HD 60435) was classified as A3 SrEu in Renson et al. (1991). Ghazaryan, Alecian \& Hakobyan (2019) report abundances for the star and classify it as neither He-poor or rich. Kochukhov \& Bagnulo (2006) detected a surface magnetic field 


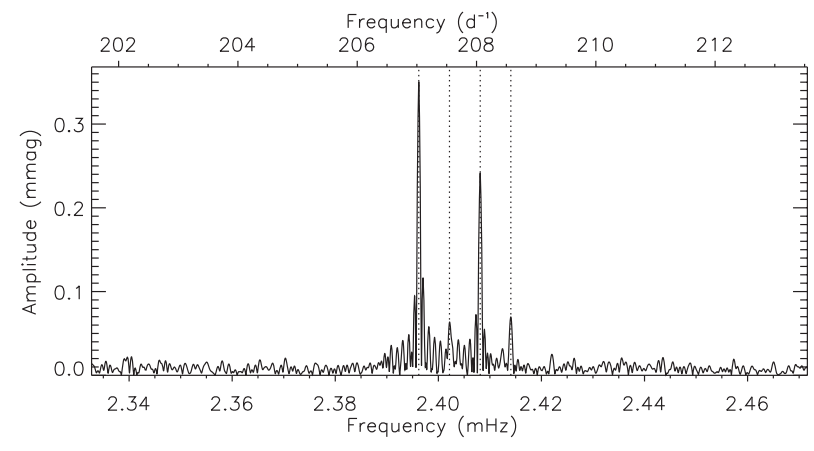

Figure 37. Amplitude spectrum of the light curve of TIC 326185137 , showing the single pulsation mode split into a multiplet. Note the different structure seen here compared with the photometric $B$ data of Kurtz et al. (1996b).

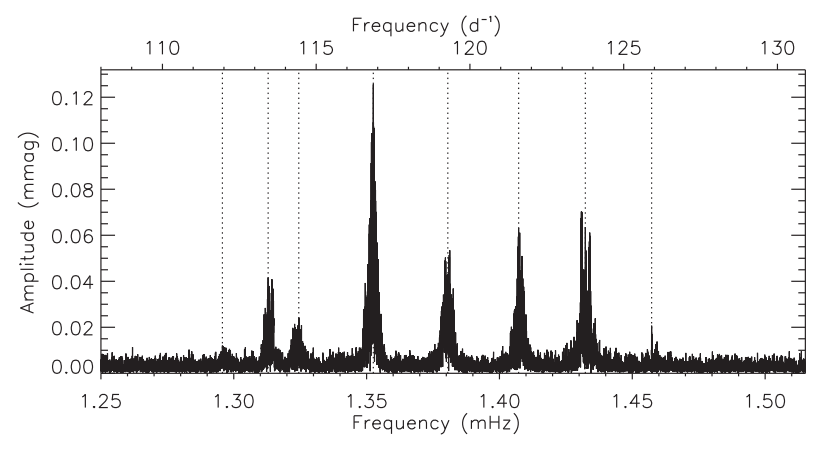

Figure 38. Amplitude spectrum of the light curve of TIC 340006157 , showing the many pulsation modes. The broad peaks indicate significant amplitude variability in this star, as reported in the literature.

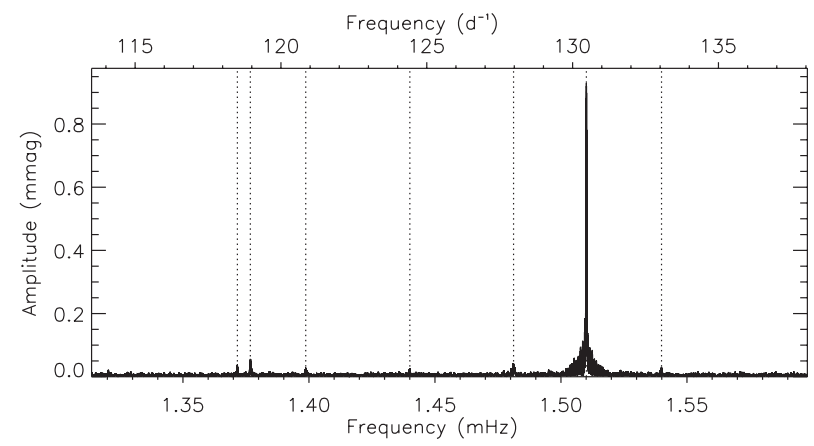

Figure 39. Amplitude spectrum of the light curve of TIC 348717688 , showing the multiple pulsation modes. All modes show signs of frequency variability.

with $B_{z}=-0.32 \pm 0.09 \mathrm{kG}$. The TIC gives $T_{\text {eff }}=8427 \mathrm{~K}$, though values in the literature range as low as $7694 \mathrm{~K}$ (McDonald, Zijlstra \& Boyer 2012). The rotation period was reported by Kurtz, van Wyk \& Marang (1990) to be $7.68 \mathrm{~d}$, with the light curve displaying a double wave of unequal depth.

The star was first identified as an roAp star by Kurtz (1984). Matthews (1987) conducted a detailed study and identified 17 frequencies, which were identified as consecutive radial overtones of $\ell=1$ and $\ell=2$ modes, with amplitudes which were highly variable on time-scales of days. Vauclair \& Théado (2004) attempted modelling based on these frequencies, with results that suggested the presence of a He gradient inside the star. Balona et al. (2019) per- formed a preliminary analysis using TESS and detected 6 independent frequencies between 1.3079 and $1.4352 \mathrm{mHz}\left(113-124 \mathrm{~d}^{-1}\right)$. All peaks were broad and poorly resolved, and many displayed variable amplitudes which both confirmed the results of Matthews (1987) and perhaps explained why not all the frequencies reported by the earlier author were detected in TESS data.

This star was observed in 7 sectors of Cycle 1, with data spanning a total of $296 \mathrm{~d}$ but with two 2 -sector gaps. To derive the rotation period here, we used a combination of the raw and pipeline corrected data since the pipeline has altered the astrophysical signal in sectors 7 and 10 for this star. We derive a rotation period of $7.6797 \pm 0.0001 \mathrm{~d}$.

The TESS amplitude spectrum is no different for this star as reported in the literature: it shows significant frequency and/or amplitude variability, making precise frequency extraction difficult. We are, however, able to identify eight pulsation frequencies in the data, which we have listed in Table 1. Some of the extracted frequencies are the same as those reported by Matthews (1987), but we do not detect the lowest frequency modes reported in that work. Only the second highest frequency mode shows rotationally split sidelobes in the amplitude spectrum. Due to the broad nature of the signal in the amplitude spectrum (Fig. 38) we have inflated our uncertainties by a factor of 100 to reflect the uncertainty in the frequency determination. This star requires a detailed individual study in the light of significant amplitude variations and short mode lifetimes.

\subsubsection{TIC 348717688}

TIC 348717688 (HD 19918) was observed in sectors 1, 12, and 13, and has a spectral type of A5 SrEuCr (Renson \& Manfroid 2009). Its pulsations were first detected by Martinez \& Kurtz (1991a) and revisited later by Martinez et al. (1995) using ground-based photometry from SAAO. In addition to the dominant pulsation frequency at $1.510 \mathrm{mHz}\left(130.5 \mathrm{~d}^{-1}\right)$ and its harmonics, a second frequency at $1.4806 \mathrm{mHz}\left(127.9 \mathrm{~d}^{-1}\right)$ was detected. TIC 348717688 has a known mean longitudinal magnetic field strength of $-0.63 \pm 0.09 \mathrm{kG}$ (Hubrig et al. 2006), although a rotation period is not currently known. A mean field modulus of $1.6 \mathrm{kG}$ was derived by Ryabchikova et al. (2007).

More recently, Cunha et al. (2019) analysed TESS sector 1 data of TIC 348717688 and extracted eight significant pulsation frequencies, including the dominant frequency originally reported by Martinez \& Kurtz (1991a). Mathys et al. (2020b), using the complete TESS Cycle 1 data set, classified this star as an ssrAp star through the lack of detection of a rotation signal, and very sharp spectral lines.

Despite the large gap in the Cycle 1 data set, the window function of the data does not significantly interfere with the frequency analysis of this star. However, we identify frequency variability in this star We are able to extract seven pulsation frequencies from the Cycle 1 light curve (Fig. 39), and note the presence of the first harmonic of the highest amplitude mode. The frequency not detected here, but quoted in Cunha et al. (2019), is likely the result of the frequency variability of a nearby mode as this mode is now split into several peaks in the amplitude spectrum.

\subsubsection{TIC 363716787}

TIC 363716787 (HD 161459) was observed in sector 13, and has a spectral type of Ap EuSrCr (Houk 1978). It has a dominant pulsation frequency of $1.39 \mathrm{mHz}\left(120.1 \mathrm{~d}^{-1}\right.$; Martinez \& Kauffmann 1990; Martinez et al. 1991). A single mean longitudinal magnetic field measurement indicates $B_{z}=-1.8 \mathrm{kG}$ (Bagnulo et al. 2015). 

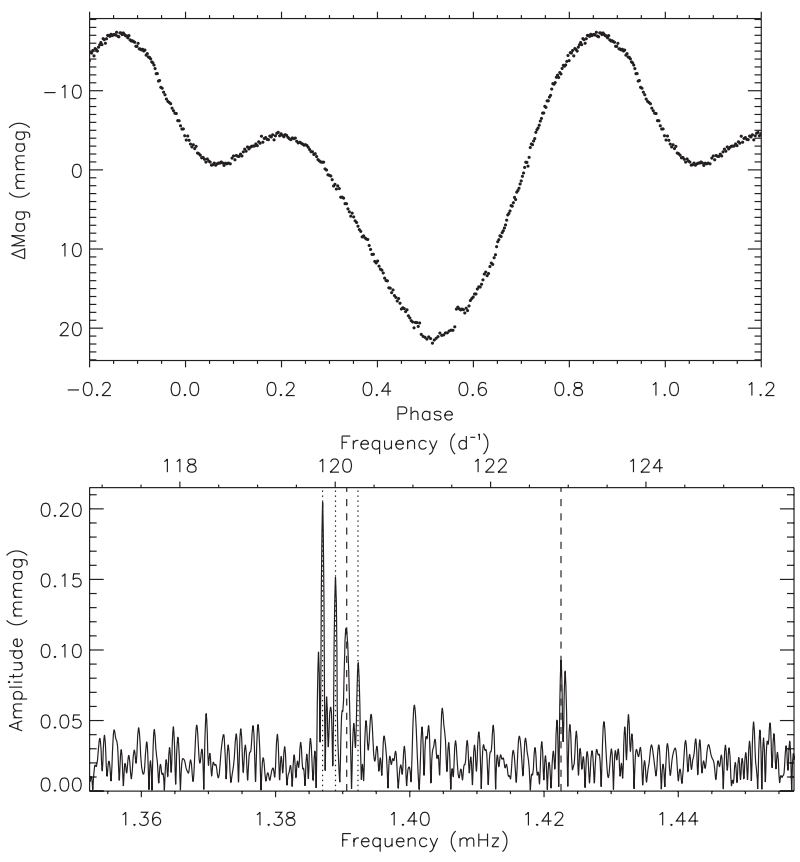

Figure 40. Top: light curve of TIC 363716787 phased on its rotation period of $5.9660 \mathrm{~d}$. Bottom: amplitude spectrum of the light curve of TIC 363716787. The dashed lines represent the two extracted modes, while the dotted lines show the multiplet components.

The TESS data allow us to provide a rotation period measurement of $5.966 \pm 0.001 \mathrm{~d}$. The folded light curve, Fig. 40, shows a double wave, implying that we see both magnetic poles, under the assumption that the chemical spots form at or near the magnetic poles. The pulsation spectrum of this star, as noted by Martinez et al. (1991), shows amplitude variability of its modes. We detect two independent modes in this star, with one showing rotationally split sidelobes. It is unclear which of the multiplet peaks is the pulsation mode, but we follow Martinez et al. (1991), taking the mode to be at $1.3909 \mathrm{mHz}\left(120.1725 \mathrm{~d}^{-1}\right)$. This mode is separated from the next by $\approx 31 \mu \mathrm{Hz}$ which is plausibly half the large frequency separation in this star.

\subsubsection{TIC 368866492}

TIC 368866492 (HD 166473) was classified as A5p SrCrEu in Renson \& Manfroid (2009). The TESS Asteroseismic Target List (ATL; Schofield et al. 2019) reports $T_{\text {eff }}=6811 \mathrm{~K}$, but this is the low end of the range of $T_{\text {eff }}$ values from the literature, which range as high as $8500 \mathrm{~K}$ (Ammons et al. 2006). Abundance analysis of this star, conducted by Gelbmann et al. (2000), indicated abundance anomalies typical of roAp stars. Mathys, Khalack \& Landstreet (2020a) used magnetic field modulus measurements to determine the rotation period to be $3836 \pm 30 \mathrm{~d}$, using high-resolution spectroscopy spanning the period 1992-2019, and confirming an earlier report of $3514 \mathrm{~d}$ by Shavrina et al. (2014). The magnetic field modulus varies from 5.6 to 8.6 kG for this star (Mathys et al. 2020a).

Kurtz \& Martinez (1987) first detected photometric oscillations in TIC 368866492, finding periods between 8.8 and 9.1 min (1.8315$1.8939 \mathrm{mHz} ; 158.2-163.6 \mathrm{~d}^{-1}$ ), while Kurtz, Elkin \& Mathys (2003) and Mathys, Kurtz \& Elkin (2007) confirmed the presence of oscillations using radial velocity measurements. Mathys et al. (2007) reported three frequencies, $1.833,1.886$, and $1.928 \mathrm{mHz}$, with am-

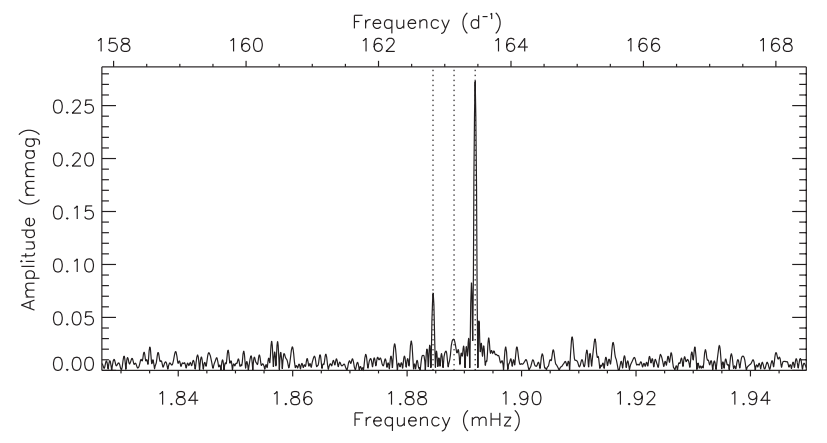

Figure 41. Amplitude spectrum of the light curve of TIC 368866492 showing the three equally split modes.

plitudes up to $110 \mathrm{~m} \mathrm{~s}^{-1}$, but all with low signal-to-noise. They also suggested that their data hinted at periodic variability of the mean magnetic field modulus, with an amplitude of $0.021 \pm 0.005 \mathrm{kG}$.

TESS observed TIC 368866492 in sector 13. As discussed by Mathys et al. (2020b), there is no sign of rotational variability in the light curve, only low-frequency noise suspected to arise from contamination. We detect three main pulsation frequencies in this star, but note there is power excess in the amplitude spectrum after they have been removed. This leads us to speculate there are further low-amplitude modes that are not fully resolved in the TESS data.

The three peaks that we do see (Fig. 41) have equal phases and are equally split by $3.69 \mu \mathrm{Hz}$ which would correspond to a rotation frequency of $3.14 \mathrm{~d}$ if the multiplet is caused by oblique pulsation. However, from the literature, we know this star to have an extremely long rotation period. A different interpretation of this splitting is either the large or small frequency separation. With a value of $3.69 \mu \mathrm{Hz}$ this is too small to be the large frequency separation, but is plausibly the small separation. However, in that situation, one would require modes of $\ell=1,3,5$ or $\ell=0,2,4$ to be visible and would need an explanation for why they would be equally split. This star needs careful consideration and modelling to solve these conundrums, which we leave to a future study.

\subsubsection{TIC 369845536}

TIC 369845536 (2MASS J19400781-4420093) is a well-studied, high-amplitude roAp star with a distorted quadrupole mode (Holdsworth et al. 2018a). It was originally discovered to have highfrequency variability by Holdsworth et al. (2014a) using SuperWASP photometry, and a dominant pulsation frequency of $2.042 \mathrm{mHz}$ $\left(176.4 \mathrm{~d}^{-1}\right)$. Spectroscopy obtained by Holdsworth et al. (2018a) led them to classify its spectral type as $\mathrm{A} 7 \mathrm{VpEu}(\mathrm{Cr})$. The groundbased photometry also allowed the rotation period to be determined as $9.5344 \pm 0.0012 \mathrm{~d}$, and modelling of the amplitude and phase modulation of its quadrupole pulsation mode confirmed its distorted nature.

TESS observed TIC 369845536 during sector 13. The data span $26 \mathrm{~d}$ which makes the determination of the rotation period less certain in the TESS data, at $9.50 \pm 0.02 \mathrm{~d}$ over the previous measurement. The pulsation signature is strikingly large in this star; it is the roAp star with the highest amplitude mode seen in $B$ photometric observations, which is also the case here with the TESS observations (Fig. 42). Beyond the known pulsation mode in this star, we detect no further modes. The multiplet structure of the $\ell=2$ mode is similar to that seen in $B$ data (Holdsworth et al. 2018a). 


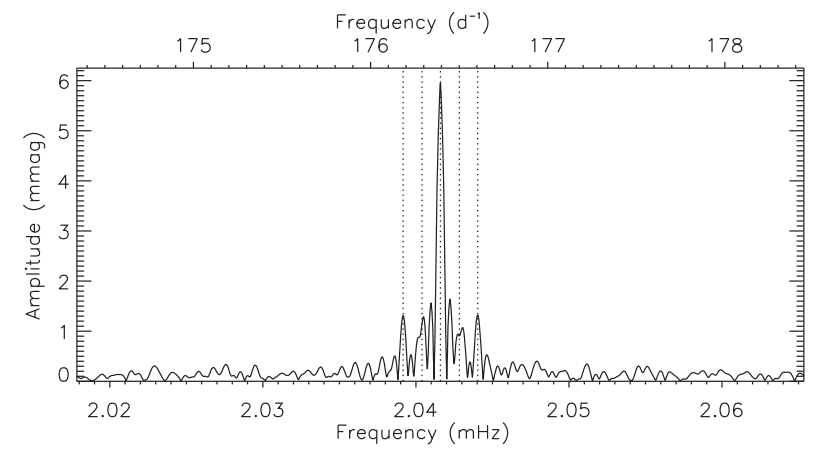

Figure 42. Amplitude spectrum of the light curve of TIC 369845536 showing the quadrupole mode with its equally split quintuplet of peaks.

\subsubsection{TIC 394124612}

TIC 394124612 (HD 218994) was observed in sector 1, and previously analysed by Cunha et al. (2019). It has a spectral type of A3 Sr (Renson \& Manfroid 2009), but is also known to be a visual binary with a separation of $1.2 \operatorname{arcsec}$ (Renson et al. 1991). Pulsations consistent with a $\delta$ Sct star and a longitudinal magnetic field strength of $0.44 \pm 0.02 \mathrm{kG}$ were detected by Kurtz et al. (2008). Despite attempts to detect roAp pulsations, none have so far been detected in photometry (see e.g. Martinez \& Kurtz 1994b). However, analysis of spectroscopic time-series data has discovered variability in its $\mathrm{Nd}$ III and Pr III lines (González et al. 2008), which showed a frequency of $1.17 \mathrm{mHz}\left(101.1 \mathrm{~d}^{-1}\right)$.

Since an analysis of the TESS data has already been presented by Cunha et al. (2019), we refer the reader to that work while providing the rotation period and pulsation frequency in Table 1 .

\subsubsection{TIC 394272819}

TIC 394272819 (HD 115226) is a well-known $\alpha^{2}$ CVn variable star. It was classified as $\mathrm{Ap} \operatorname{Sr}(\mathrm{Eu})$ by Houk \& Cowley (1975). The $T_{\text {eff }}$ values in the literature for this star are mostly in agreement, with a value around $7640 \mathrm{~K}$ (Kochukhov \& Bagnulo 2006; Smalley et al. 2015). Using this value and the Hipparcos parallax, Kochukhov \& Bagnulo (2006) determine $L=7.2 \pm 1.9 \mathrm{~L}_{\odot}$. Later, Kochukhov et al. (2008) determined the projected rotational velocity $v \sin i=$ $25-30 \mathrm{~km} \mathrm{~s}^{-1}$ and conducted an abundance analysis, finding nearly solar abundances of $\mathrm{Mg}, \mathrm{Si}, \mathrm{Ti}$, and $\mathrm{Fe}$, an overabundance of $\mathrm{Cr}, \mathrm{Co}$, and rare earth elements, and an underabundance of Ba. Bagnulo et al. (2015) reported a $\left\langle B_{z}\right\rangle_{\text {rms }}$ value of $0.828 \mathrm{kG}$.

The roAp nature of TIC 394272819 was discovered by Kochukhov et al. (2008). Using time-series high-resolution HARPS spectroscopy, they measured the high-amplitude $10.87-\min (1.5333 \mathrm{mHz}$; $132.5 \mathrm{~d}^{-1}$ ) oscillation in this star from radial velocity variations. Pulsational variability was discovered in the cores of hydrogen lines and in doubly ionized Pr, Nd, and Dy lines. Recently, the same 102 HARPS spectra were analysed by Binnenfeld, Shahaf \& Zucker (2020) by using the unit-sphere representation periodogram (USURPER), finding the same period.

Photometric ASAS-3 data of TIC 394272819 were analysed by Hümmerich, Paunzen \& Bernhard (2016). They detected a clear signal with a period of $2.9882 \mathrm{~d}$. In addition they checked the Hipparcos data and found marginal variability with $P=3.61 \mathrm{~d}$, which is not present in the ASAS-3 data. Joshi et al. (2016) confirmed the presence of 10.86-min oscillations analysing high-speed photometry from the Nainital-Cape Survey.

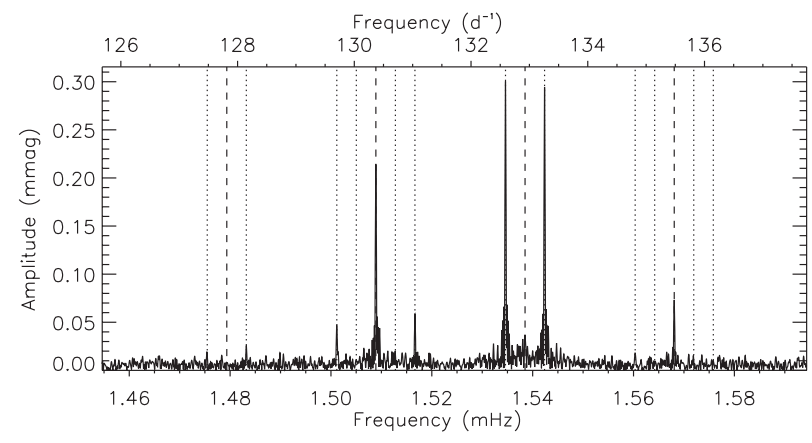

Figure 43. Amplitude spectrum of the light curve of TIC 394272819 showing the multiple modes in this star. We mark the presumed modes with dashed lines, with rotationally split sidelobes marked by dotted lines.

TIC 394272819 was observed during sectors 11 and 12. The combined PDC_SAP data allow us to derive a rotation period of $2.98827 \pm 0.00008 \mathrm{~d}$ which is consistent with the ASAS-3 data. There is no evidence of the 3.61-d signal found in the Hipparcos data. We detect four pulsation modes in this star. In increasing frequency we see a singlet, three components of a quintuplet, a triplet, and another singlet. Fig. 43 shows the amplitude spectrum of this star. We interpret this pattern as a series of alternating $\ell=$ 1 and $\ell=2$ modes with some components lost in the noise. This interpretation is reinforced when considering the spacing between the modes; under our assumption they are all separated by $29.58 \mu \mathrm{Hz}$ which we understand to be the half of the large frequency separation for this star.

\subsubsection{TIC 402546736}

TIC 402546736 (HD 128898; $\alpha$ Cir) has many similar classifications in the literature. Here we quote that of Gray et al. (2006): A7 $\mathrm{Vp} \mathrm{SrCrEu}$. This star is a well studied, naked eye object, and as such has a plethora of information in the literature. We provide here a very limited introduction to the star, and encourage the interested reader to explore the references provided.

Effective temperature measurements for TIC 402546736 range from $7420 \mathrm{~K}$ (e.g. Bruntt et al. 2008; Desidera et al. 2015) to around 8000 K (e.g. Kurtz \& Martinez 1993; Matthews, Kurtz \& Martinez 1999) derived through a variety of different methods. $\log g$ determinations also vary, but are consistently around $4.1\left(\mathrm{~cm} \mathrm{~s}^{-2}\right)$ within the quoted errors (e.g. Kochukhov, Shulyak \& Ryabchikova 2009b; Sikora et al. 2019a), with $v \sin i$ values consistently around $13 \mathrm{~km} \mathrm{~s}^{-1}$ (e.g. Kupka et al. 1996; Ammler-von Eiff \& Reiners 2012). The abundance pattern in TIC 402546736 is typical for Ap stars (Kupka et al. 1996; Bruntt et al. 2008), with underabundances of C, $\mathrm{N}$, and $\mathrm{O}$, and overabundances of rare-earth and some other heavy elements. Kochukhov et al. (2009b) built a self-consistent empirical model atmosphere of TIC 402546736 and conducted stratification modelling of some elements, showing that chemical stratification has an important effect on the model structure of TIC 402546736. A $\langle B\rangle$ value of $0.9 \mathrm{kG}$ have been reported for this star (Bruntt et al. 2008). Sikora et al. (2019b) derived a dipolar field strength of $1.4 \mathrm{kG}$ from the analysis of mean longitudinal field measurements.

TIC 402546736 was classified as an roAp star showing a 6.8-min ( $2.4510 \mathrm{mHz} ; 211.8 \mathrm{~d}^{-1}$ ) oscillation by Kurtz \& Cropper (1981) and subsequently, 47 hours of high-speed, ground-based photometry was analysed by Kurtz, Allen \& Cropper (1981). Notable results from studies of this star are those of Kurtz et al. (1994a) who found the 

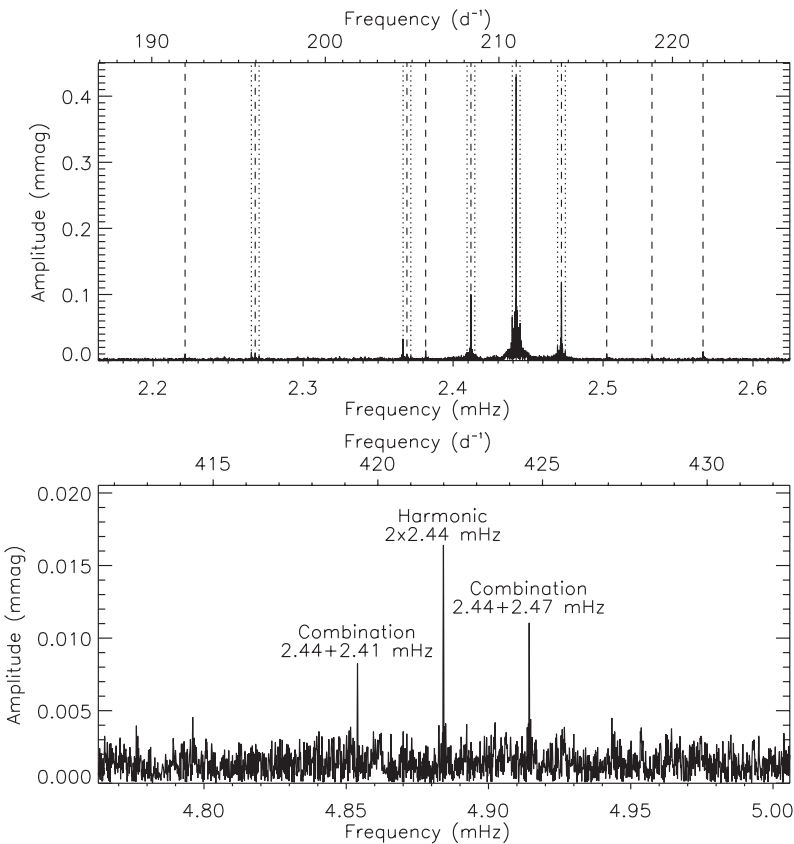

Figure 44. Amplitude spectrum of the SAP light curve of TIC 402546736 $(\alpha \mathrm{Cir})$ showing the multiple modes in this star. The top panel shows the region where the pulsation modes are detected. We mark the modes with dashed lines, with rotationally split sidelobes marked by dotted lines. The bottom panel shows the harmonic of the dominant mode, and two additional peaks that are the result of non-linear interactions in this star. Note the change in scale between the two panels.

star to pulsate in a dipole mode with a frequency of $2.442 \mathrm{mHz}$ $\left(211.0 \mathrm{~d}^{-1}\right)$, and subsequent lower amplitude modes. They also provided a precise rotation period of $4.4790 \mathrm{~d}$. Later, Bruntt et al. (2009) used $84 \mathrm{~d}$ of WIRE observations to refine the separation of the modes in this star to be $30.2 \mu \mathrm{Hz}$. Weiss et al. (2016) and Weiss et al. (2020) used 4 BRITE satellites to observe TIC 402546736 for a total of $146 \mathrm{~d}$, providing them data to model the spot configuration of the star, and confirm the previously tentative detection of some low amplitude modes.

Beside the photometric observations of TIC 402546736, there have been substantial spectroscopic studies using high time resolution spectra. These studies (e.g. Baldry et al. 1998a, 1999; Kochukhov \& Ryabchikova 2001; Balona \& Laney 2003; Ryabchikova et al. 2007) have shown that the principal pulsation mode can have an amplitude up to $1 \mathrm{~km} \mathrm{~s}^{-1}$, with some spectral lines pulsating in antiphase with others, suggesting a node, or false node, in the atmosphere of the star (Sousa \& Cunha 2011; Quitral-Manosalva et al. 2018).

TESS observed TIC 402546736 during sectors 11 and 12. We use the SAP data to determine a rotation period of $4.4812 \pm 0.0005 \mathrm{~d}$ which is different from that of Kurtz et al. (1994a) by $4.5 \sigma$. We also use the SAP data for the pulsation analysis that follows, since the SPOC pipeline has introduced significant noise to this bright star. As expected from the literature, the amplitude spectrum is rich in pulsation modes and rotationally split sidelobes (Fig. 44). We extract 23 significant peaks from the amplitude spectrum, but note there is evidence of further modes seemingly present in the data. Of those 23 peaks, we identify 10 as pulsation modes (see Table 1), one as a harmonic and 2 as peaks due to non-linear interactions. The latter 3 peaks are all found above the Nyquist frequency and are reminiscent of those seen in the K2 data of 33 Lib (HD 137949; Holdsworth et al. 2018c), making TIC 402546736 only the second roAp star to show such non-linear interactions.

Although not all modes are separated by the same frequency, many are separated by $30.18 \mu \mathrm{Hz}$ which we take to be half of the large frequency separation in this star, as did Bruntt et al. (2009). For those modes where the frequency spacing is not a multiple of $30.18 \mu \mathrm{Hz}$, we postulate that these mode frequencies have been affected by the magnetic field, as was seen in TIC 279485093 (HR 1217; Kurtz et al. 2005).

\subsubsection{TIC 434449811}

TIC 434449811 (HD 80316) was classified as $\mathrm{ApSr}(\mathrm{Eu}$ ?) with 'extremely strong Sr II' by Bonsack (1974). There is no detailed spectroscopic analysis of the fundamental properties in the literature, so $T_{\text {eff }}$ values are based on photometry with values ranging from 8110 K (Gaia Collaboration 2018) to $9120 \mathrm{~K}$ (Ammons et al. 2006). Using $u v b y \mathrm{H} \beta$ from Paunzen (2015), and the calibrations of Moon $\&$ Dworetsky (1985), gives $T_{\text {eff }}=8420 \mathrm{~K}$, which is consistent with $8280 \mathrm{~K}$ obtained from the spectral energy distribution (McDonald et al. 2012). The mean longitudinal magnetic field strength has been determined to be $-0.18 \pm 0.04 \mathrm{kG}$ by Hubrig et al. (2006) and $-0.25 \pm 0.09 \mathrm{kG}$ by Bagnulo et al. (2015).

The rotation period was determined to be $2.09 \mathrm{~d}$ (Manfroid \& Mathys 1986; Kurtz et al. 1997b), although a recent value of $1.91 \mathrm{~d}$ was given by Oelkers et al. (2018). With $v \sin i=32 \pm 5 \mathrm{~km} \mathrm{~s}^{-1}$ Wade (1997) estimated a rotational axis inclination of $i=60 \pm 15^{\circ}$. The radial velocity is given as $9.5 \pm 1.1 \mathrm{~km} \mathrm{~s}^{-1}$ by Gontcharov (2006). However, five measurements with HARPS show a large variation over a 2 -yr time-span, ranging from 10.6 to $46.7 \mathrm{~km} \mathrm{~s}^{-1}$ (Trifonov et al. 2020), indicating this could be a spectroscopic binary star. The star is part of a very wide (>70 arcsec) optical pair with HD 80298 (BD-192673; WDS J09184-2022A; Mason et al. 2001), but there is no physical association.

TIC 434449811 is a known roAp star, with a $7.4-\min (2.2523 \mathrm{mHz}$; $194.6 \mathrm{~d}^{-1}$ ) oscillation discovered by Kurtz (1990) in photometric observations. A later, more in depth photometric study was presented by Kurtz et al. (1997b) who found a frequency triplet with equal amplitudes in a $B$ filter. Elkin et al. (2008) found a pulsation signal of the same period with an amplitude of $0.32 \mathrm{~km} \mathrm{~s}^{-1}$ in spectral lines.

TESS observed the star during sector 8. The PDC_SAP data, after the removal of some outlying points at the start of the second orbit, provide a rotation period for this star of $2.08862 \pm 0.00004 \mathrm{~d}$, which is consistent with the literature value. After removal of the rotation signature and its harmonics, there are two peaks remaining in the $\delta$ Sct frequency range (Fig. 45). It is unclear whether these are mode frequencies in TIC 434449811 or in a companion star. Given the relatively weak magnetic field in this star, it is possible that the $\delta$ Sct frequencies are in the Ap star (Murphy et al. 2020), but detailed modelling and the exclusion of other possibilities is required.

The roAp pulsation signature in this star has a different multiplet structure than previously seen (Kurtz et al. 1997b); there are, in the TESS data, two peaks with similar amplitudes, and a further 3 peaks with low signal-to-noise. This change in multiplet structure is similar to that seen in TIC 326185137 (HD 6532; Kurtz \& Holdsworth 2020) and may be a result of the different filters probing different depths in the stellar atmosphere. A detailed analysis of this star is required to fully understand its pulsation properties. 

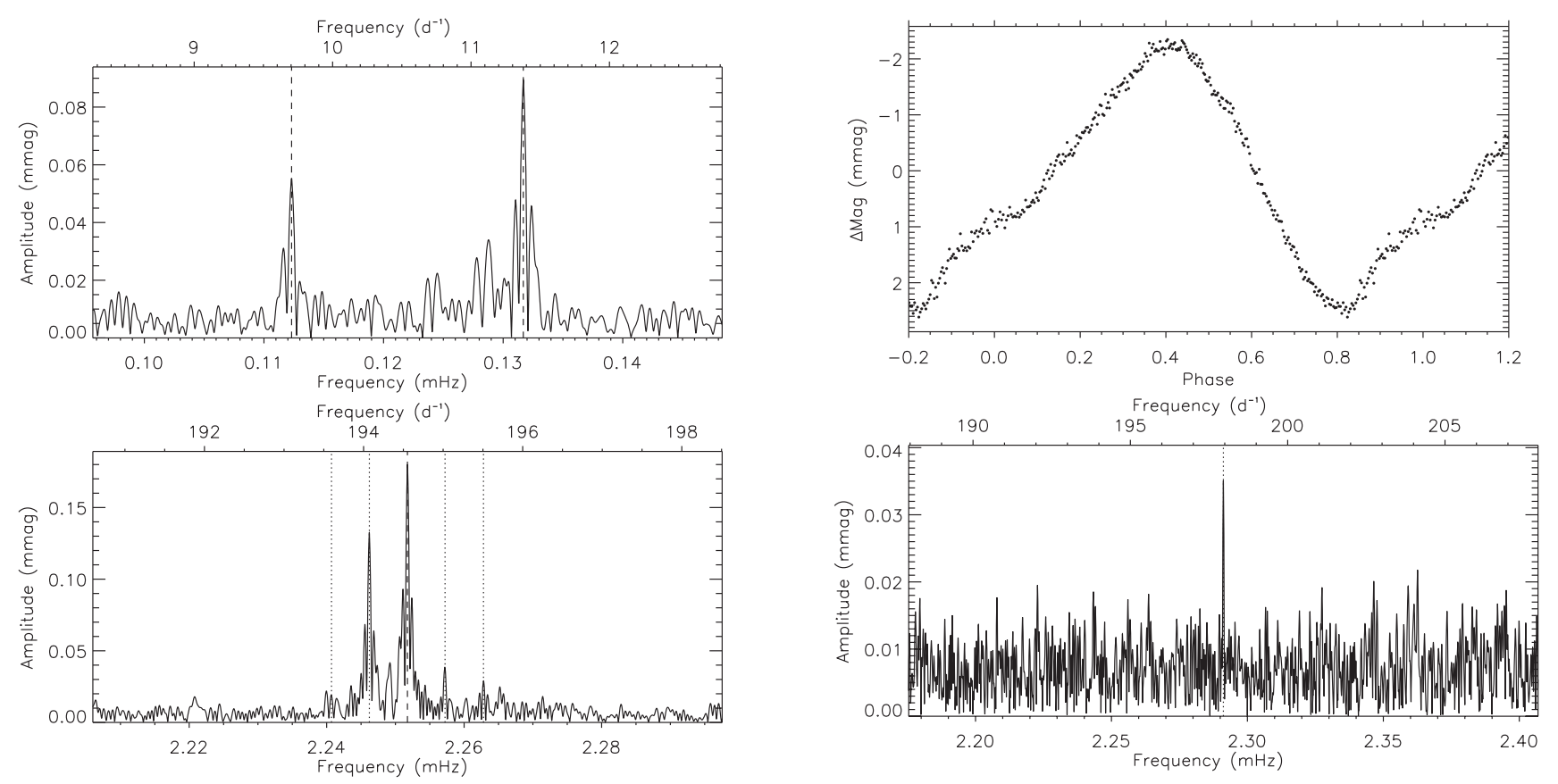

Figure 45. Amplitude spectrum of the light curve of TIC 434449811. The top panel shows the low-frequency modes found in this star, with the bottom panel showing the multiplet around the known pulsation mode.

\subsubsection{TIC 469246567}

TIC 469246567 (HD 86181) is listed as an $\alpha^{2}$ CVn star on SIMBAD and has a spectral type ApSr (Houk \& Cowley 1975). Kurtz \& Martinez (1994) discovered 6.2-min $\left(2.6882 \mathrm{mHz} ; 232.3 \mathrm{~d}^{-1}\right)$ oscillations in TIC 469246567, making it one of the most rapidly oscillating Ap stars. A longitudinal magnetic field strength of $536 \pm 58 \mathrm{kG}$ was measured by Bagnulo et al. (2015). Despite the $\alpha^{2} \mathrm{CVn}$ classification, there is only one recent measure of the rotation period of this star (Shi et al. submitted).

TIC 469246567 was observed by TESS during sectors 9 and 10 . These data have been extensively analysed by Shi et al. (submitted) so we refer the reader there to a detailed discussion. In short, the pulsation spectrum consists of alternating dipole, quadrupole, and dipole modes, all rotationally split into multiplets. The TESS data also provide the first measure of the rotation period of this star to be $2.05115 \pm 0.00006 \mathrm{~d}$. The pulsation frequencies, and rotation period, are included in Table 1 for completeness.

\section{CANDIDATE ROAP STARS}

In this section, we provide information on a number of stars which were flagged by the search teams as possible roAp stars. These stars are classed as candidate roAp stars since either their spectral classification does not indicate chemical peculiarity, their pulsation signature does not show signs of multiplets split by the rotation frequency, or their presumed pulsation signal is close to a signal-tonoise limit of 4.0.

\subsection{TIC 1727745}

TIC 1727745 (HD 113414) was classified as an F7/8V type star by Houk \& Smith-Moore (1988). There have been numerous studies that attempt to calculate physical parameters of the star. Among these, Haakonsen \& Rutledge (2009) identified the star as an X-

Figure 46. Top: phase folded light curve of TIC 1727745, phased on a period of $3.172 \mathrm{~d}$. Bottom: the high frequency pulsation mode found in this star.

ray source and Suchkov, Makarov \& Voges (2003) found its X-ray luminosity to be $\log L_{\mathrm{x}}=29.45 \mathrm{erg} \mathrm{s}^{-1}$ in the investigation of X-ray properties of the ROSAT F stars. Suchkov et al. (2003) also estimated the temperature and metallicity to be $T_{\text {eff }}=6200 \mathrm{~K}$ and Fe/H $=0.04$, respectively.

Using precision radial velocities from HIRES on the Keck I telescope, Butler et al. (2017) stated that the star showed chromospheric emission variations, which led to radial velocity variations, due to either stellar rotation or long-term activity cycles. Additionally, $(b-$ $y$ ), $m_{1}, c_{1}$ colour indexes and $\mathrm{H} \beta$ index of the star were provided to be 0.357, 0.182, 0.386, and 2.624, respectively (Hauck \& Mermilliod 1998; Paunzen 2015), which do not imply this star is chemically peculiar.

Observed in sector 10, the TESS data show a rotationally modulated light curve, which has a period of $3.172 \pm 0.001 \mathrm{~d}$ (Fig. 46). The well-defined phased light curve suggests that the surface inhomogeneities are stable over the observations period. The pulsation signal in the star occurs at $2.29116 \pm 0.00004 \mathrm{mHz}\left(197.956 \pm 0.004 \mathrm{~d}^{-1}\right)$ which is in the frequency range of known roAp pulsations.

The contamination factor, in the TIC, for this star is very small (0.0001) which implies all of the light in the photometric aperture is originating from the target star.

\subsection{TIC 3814749}

TIC 3814749 (HD 3748) was also listed in both Balona et al. (2019) and Kobzar (2020) as an roAp candidate. The star is a known double system (with a contamination factor of 1.431) which have spectral types of A0/1 IV/V and A9/F0V (Houk \& Swift 1999b), but with also A5 quoted in the literature. We obtained a classification spectrum from SAAO and confirm the lack of strong chemical peculiarities in this star, suggesting it is not an Ap star (Fig. A13). However, the $\mathrm{Ca}$ II $\mathrm{K}$ line is weak in the star, as is the $\mathrm{Mg}$ II $4481 \AA$ line.

TESS observed TIC 3814749 during sector 3 . With those data, we detect a low frequency signal corresponding to a period of 

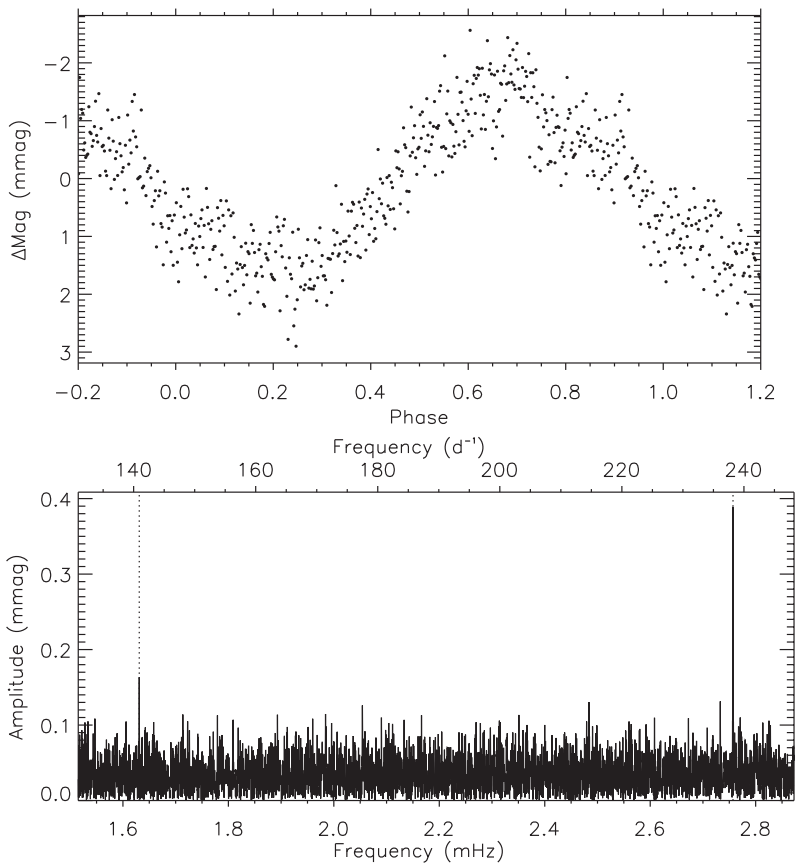

Figure 47. Top: phase folded light curve of TIC 3814749, phased on a period of $1.689 \mathrm{~d}$. Bottom: the two high frequency peaks found in this star, as marked by dotted lines.

$1.689 \pm 0.002 \mathrm{~d}$. We also find two peaks at higher frequency which we assume are a result of pulsational variability (Fig. 47), although the lower frequency peak is at low significance. These two peaks are well separated in frequency, by $1.12 \mathrm{mHz}$, which requires a significant range of unexcited overtones as has been seen in TIC 139191168 (HD 217522) and now also in TIC 49332521 (HD 119027).

However, the star is listed as an Algol type binary (Samus' et al. 2003). It is possible that the low frequency signal is the orbital frequency of the A star and a compact companion. In this case, a large gap between the observed frequencies is less uncommon (e.g. Sahoo et al. 2020). High-resolution spectroscopic monitoring of this system is needed to solve this problem.

\subsection{TIC 158637987}

There is little information in the literature about TIC 158637987 (HD 10330). Houk \& Cowley (1975) gave the spectral type as A9V, indicating a chemically normal star. Paunzen (2015) provided Strömgren-Crawford photometric indices of $b-y=0.207, m_{1}=$ $0.143, c_{1}=0.702$, and $\mathrm{H} \beta=2.732$. Using the calibrations of Crawford (1979), these indices suggest the star is significantly metal weak.

The TESS observations, collected during sectors 2 and 3, show significant variability in the low-frequency range which we take to be $g$ mode pulsations (Fig. 48). The high-frequency variability in this star occurs at about $2.71 \mathrm{mHz}\left(236 \mathrm{~d}^{-1}\right.$; Fig. 48), where we identify two peaks of marginal significance. The separation of these two peaks $\left(0.01642 \mathrm{mHz} ; 1.4187 \mathrm{~d}^{-1}\right)$ is close to, but not the same as, a peak in the low frequency range $\left(0.01713 \mathrm{mHz} ; 1.4797 \mathrm{~d}^{-1}\right)$, thus we cannot deduce whether the peaks are split by the stellar rotation frequency. Spectroscopic observations are required of this star to test the existence of chemical peculiarities and/or a magnetic field. If either exist, this star provides another example of a $\gamma$ Dor-roAp
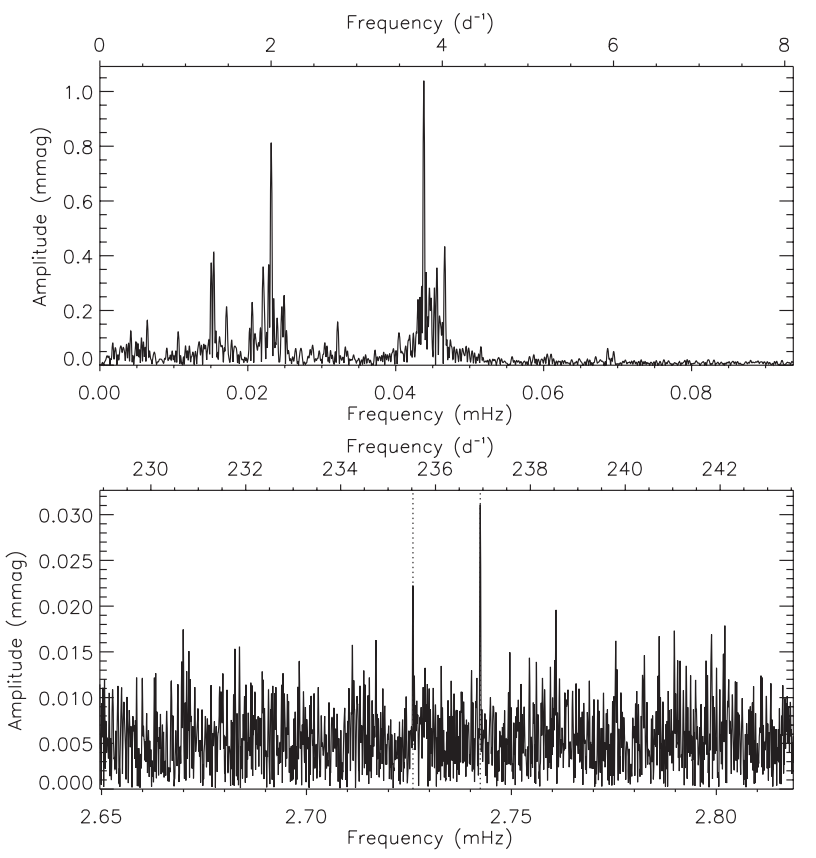

Figure 48. Top: $g$-mode frequencies seen in TIC 158637987. Bottom: the two pulsation modes found in this star, as marked by dotted lines.

hybrid star. If this is an roAp star, it is unique in having apparently low metallicity.

The TIC provides a contamination factor of 0.048 for TIC 158637987, suggesting there is very little contamination from a nearby (on the sky) star.

\subsection{TIC 324048193}

TIC 324048193 (HD 85892) was classified as Ap Si by Houk \& Cowley (1975). The literature provides $T_{\text {eff }}$ values of between $7770 \mathrm{~K}$ (Bai et al. 2019) to $10110 \mathrm{~K}$ (Anders et al. 2019), but with most estimates around $9300 \mathrm{~K}$ (Ammons et al. 2006; McDonald et al. 2012; Chandler, McDonald \& Kane 2016). The TIC estimate is by far the highest temperature estimate at $11950 \mathrm{~K}$, and we treat that with caution. The presence of $\mathrm{Si}$ in the stellar spectrum suggests a late B early A star which is consistent with the higher temperature range. The mass and radius estimates for the star, $5.2 \mathrm{M}_{\odot}$ and $4.5 \mathrm{R}_{\odot}$ (McDonald et al. 2017; Kervella et al. 2019), suggest it has evolved away from the zero-age main sequence. A $v \sin i$ value of $45 \mathrm{~km} \mathrm{~s}^{-1}$ was quoted by Renson \& Manfroid (2009), with Watson et al. (2006) measuring a rotation period of $4.295 \mathrm{~d}$.

TESS observed TIC 324048193 during sectors 11 and 12. The light curve shows clear modulation due to spots, as expected for a magnetic $\mathrm{CP}$ star. From the variations, we derive a rotation period of $4.2953 \pm 0.0001 \mathrm{~d}$ which is consistent with that in the literature. The pulsation signal in this star is at a high frequency of $3.15347 \pm 0.00002 \mathrm{mHz}\left(272.460 \pm 0.002 \mathrm{~d}^{-1}\right.$; Fig. 49). This is among the highest frequencies known in the roAp stars. This star is also at the upper temperature range of the known roAp stars, after TIC 123231021 (KIC 7582608; Holdsworth et al. 2014b), but it is well within the theoretical instability strip for this class of pulsator (Cunha 2002).

We classify this as a candidate roAp star since the signal is detected at marginal significance ( $\mathrm{S} / \mathrm{N}=5.4$ ), and lacks signs of rotationally split sidelobes (which would be lost in the noise). Further TESS observations of this star are due, which will hopefully allow this star 


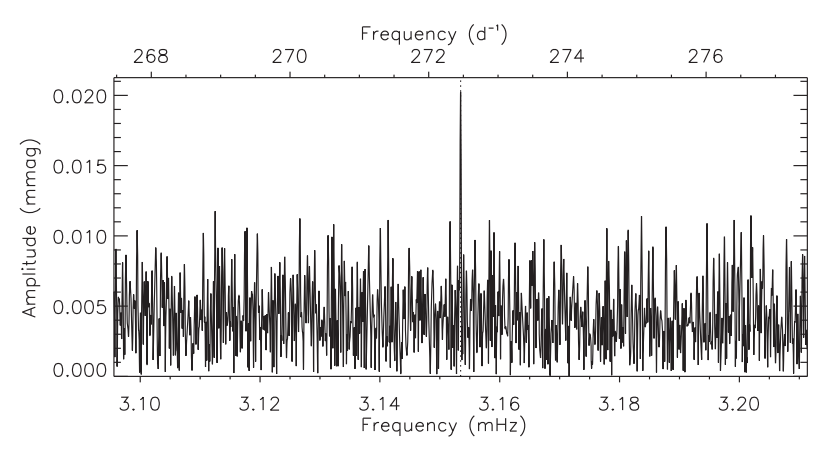

Figure 49. Suspected pulsation mode in TIC 324048193.
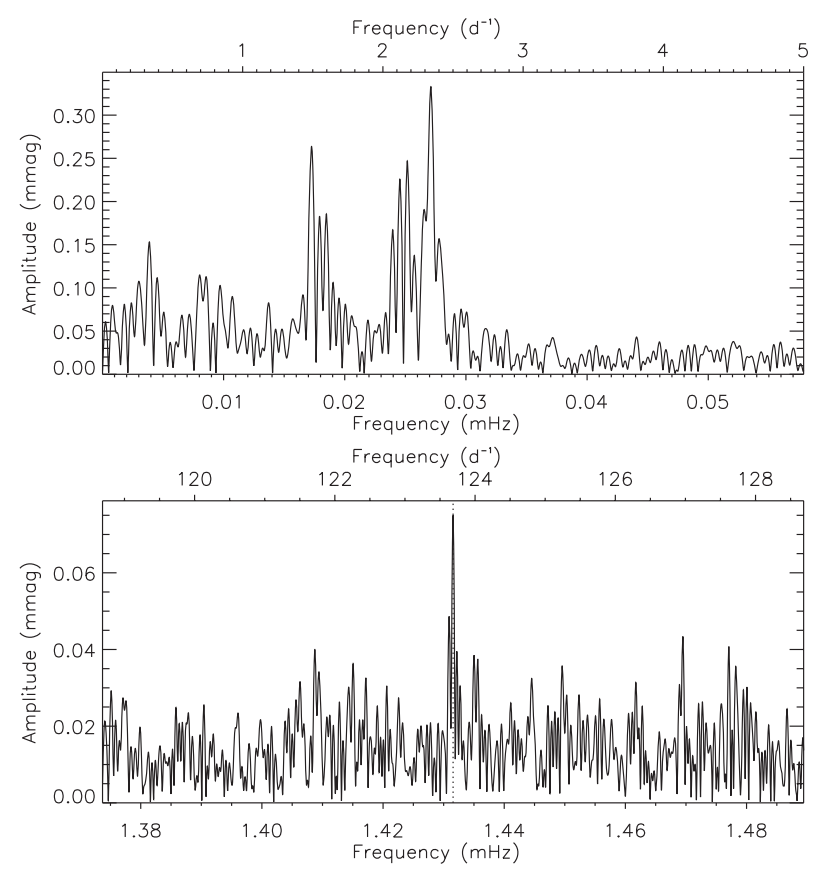

Figure 50. Top: suspected $g$-mode pulsations in TIC 410163387. Bottom: suspected roAp pulsation mode in TIC 410163387.

to be confirmed as a new roAp star. Since the contamination factor is small (0.0032) we are confident that the signal is from this star.

\subsection{TIC 410163387}

TIC 410163387 (HD 76279) was classified as an Ap SrEuCr star by Houk \& Smith-Moore (1988), although they note it as a 'weak case'. There are differing $T_{\text {eff }}$ values in the literature, with most in the region of $7100 \mathrm{~K}$ (e.g. Ammons et al. 2006; Bai et al. 2019). There has been one previous published attempt to find pulsations in this star, however the authors returned a null result at a magnitude limit of 0.8 mmag in 2-h of photometric $B$ observations (Nelson \& Kreidl 1993).

TESS observed TIC 410163387 in sector 8 . An amplitude spectrum of the light curve at low frequency (Fig. 50) reveals the presence of probable $g$-mode pulsations in this star. At high frequency, we detect a single peak at $1.43155 \pm 0.00003 \mathrm{mHz}\left(123.686 \pm 0.003 \mathrm{~d}^{-1}\right)$ which has an $\mathrm{S} / \mathrm{N}$ of 7.0. We cannot conclude, with confidence, that this peak is a true pulsation mode, and, coupled with the presence of the $g$-modes, we rate this star as a candidate roAp star until further observations are made to confirm the existence of the mode. We also note that the TIC contamination factor for this star is non-negligible at 0.01 ,implying that some of the signals may be from a contaminant source.

These factors led us to obtain a high-resolution spectrum of TIC 410163387 with SALT/HRS. The single spectrum reveals this star to be a spectroscopic binary of two components of similar temperature given the relative depths of the $\mathrm{H}_{\alpha}$ absorption line (Fig. A14). We suspect that the two pulsation signals are therefore originating on two separate stars, but confirmation of the high frequency variability is still required.

\section{CONCLUSIONS}

We have presented the results of a systematic survey of hot (>6000 K) stars observed during the first Cycle of TESS observations, covering the Southern Ecliptic hemisphere, in 2-min cadence. We selected over 50700 stars in this temperature range, and searched for pulsational variability with frequencies greater than $0.52 \mathrm{mHz}$, resulting in over 6713 detections. After the removal of obvious contaminant stars, the final sample consisted of 163 stars for detailed frequency analysis. The main discoveries from this work can be summarized as follows:

(i) We have reported the discovery of 12 new roAp stars. Six of these stars show multiple pulsation frequencies in their light curves, making them of utmost importance for asteroseismic modelling. In addition to these stars, another (TIC 119327278) shows the presence of a $\delta$ Sct mode, suggesting this star belongs to the rare $\delta$ Sct-roAp hybrid stars. This star also shows the shortest rotation period among the class of roAp stars, at $1.085 \mathrm{~d}$. We have identified the roAp star with the lowest frequency pulsation, TIC 356088697, which shows a single pulsation mode at $0.646584 \mathrm{mHz}(P=25.8 \mathrm{~min})$, allowing us to test the lowest end of the radial orders excited in the roAp stars. Of the 12 new roAp stars, we provided the first measures of the rotation period for five, and find three to show no rotational variability.

(ii) We have presented the results of TESS observations of a further 44 roAp stars that were known in the literature. Of these 44, we find three (TIC 35905913-HD 132205, TIC 93522454- HD 143487, and TIC 173372645-HD 154708) that show no clear pulsation signal in the high-precision TESS data and note that these three stars were discovered to have low amplitude pulsations through the analysis of time-resolved high-resolution spectroscopic observations. Furthermore, we present the tentative detection of modes in 2 other roAp stars which were discovered through spectroscopic observations.

(iii) Of the entire sample of 56 confirmed roAp stars observed, 68 percent (38) show rotationally modulated light curves, with rotation periods in the range $1.085-21$ (or 42) $\mathrm{d}$. It is not yet clear if there is a causal link between rotation and pulsation, but these observations will provide new results to test this relationship.

(iv) There are many instances where the rotationally split multiplets show different structures, in the same star, for the same degree mode (e.g. TIC 176516923-HD 38823, TIC 310817678-HD 88507, TIC 259587315-HD 30849). This provides a problem for the application of the oblique pulsator model where the stellar inclination angle, $i$, and the magnetic obliquity, $\beta$, can be derived or constrained. This, coupled with the difference in structure between observations made in different filters (e.g. TIC 326185137-HD 6532; Kurtz \& Holdsworth 2020), and spectral lines (e.g. TIC 280198016 HD 83368; Kochukhov 2006), provides a significant challenge in understanding the results of the oblique pulsator model.

(v) We have reported the presence of non-linear interactions in the pulsation spectrum of TIC 402546736 (HD 128898) around the 
harmonic of the principal mode. This is only the second star (after 33 Lib; Holdsworth et al. 2018c) where this phenomenon is seen.

The results presented here have provided new insights into the roAp class of pulsationally variable stars. We have identified new pulsation frequencies in many stars, and shown frequency variability is a common feature of the roAp stars. These observational results will drive forward the development of state-of-the-art theoretical models of these stars, providing insight into the physical processes at play in these magnetic, chemically peculiar stars. Puzzling frequency separations, high-frequency modes, and a lack of roAp stars probing the blue edge of the instability strip, mean these results will have a significant impact on the theoretical understanding of the rapidly oscillating Ap stars. We will use these results to conduct a homogeneous study of the properties of roAp stars and explore any correlations between stellar and pulsation properties, and investigate any differences between the observed frequencies and those predicted by models.

\section{ACKNOWLEDGEMENTS}

We thank the anonymous referee for a careful reading of the manuscript. DLH acknowledges financial support from the Science and Technology Facilities Council (STFC) via grant ST/M000877/1. MSC acknowledges the support by FCT/MCTES through the research grants UIDB/04434/2020, UIDP/04434/2020, and PTDC/FIS-AST/30389/2017, and by FEDER - Fundo Europeu de Desenvolvimento Regional through COMPETE2020 - Programa Operacional Competitividade e Internacionalização (grant: POCI01-0145-FEDER-030389). MSC is supported by national funds through FCT in the form of a work contract. VA was supported by a research grant (00028173) from VILLUM FONDEN. Funding for the Stellar Astrophysics Centre is provided by The Danish National Research Foundation (Grant agreement no.: DNRF106). The research leading to these results has received funding from the Research Foundation Flanders (FWO) by means of a senior postdoctoral fellowship to DMB with grant agreement No. $1286521 \mathrm{~N}$. This project has been supported by the Lendület Program of the Hungarian Academy of Sciences, project No. LP2018-7/2020 and by the EU's MW-Gaia COST Action (CA18104). ASG acknowledges financial support from the Max Planck Society under grant 'Preparations for PLATO science' and from ALMA-CONICYT under grant \#31170029. ZsB acknowledges the support by the János Bolyai Research Scholarship of the Hungarian Academy of Sciences. LFM acknowledges the financial support from the UNAM under grant PAPIIT IN100918. CCL acknowledges the support of the Natural Sciences and Engineering Research Council of Canada (NSERC). DM acknowledges the support of the National Astronomical Research Institute of Thailand (NARIT). JPG acknowledges funding support from Spanish public funds for research from project PID2019-107061GB-C63 from the 'Programas Estatales de Generación de Conocimiento y Fortalecimiento Científico y Tecnológico del Sistema de I+D+i y de I+D+i Orientada a los Retos de la Sociedad', and from the State Agency for Research through the 'Center of Excellence Severo Ochoa' award to the Instituto de Astrofísica de Andalucía (SEV2017-0709), all from the Spanish Ministry of Science, Innovation and Universities (MCIU). TRY acknowledges support from the NSF REU program, grant number PHY-1359195. The material is based upon work supported by NASA under award number 80GSFC21M0002. AD was supported by the ÚNKP-20-5 New National Excellence Program of the Ministry of Human Capacities and the János Bolyai Research Scholarship of the Hungarian Academy of Sciences. AD would like to thank the City of Szombathely for support under Agreement No. 67.177-21/2016. AGH acknowledges support from 'FEDER/Junta de Andalucía-Consejería de Economía y Conocimiento' under project E-FQM-041-UGR18 by Universidad de Granada and from Spanish public funds (including FEDER funds) for research under project ESP2017-87676-C5-2-R. ARB acknowledges funding support from Spanish public funds for research through the research grant PRE2018-084322, through projects ESP2017-87676-C5-5-R and PID2019-107061GB-C63, and from the State Agency for Research through the 'Center of Excellence Severo Ochoa' award to the Instituto de Astrofísica de Andalucía (SEV-2017-0709), all from the Spanish Ministry of Science, Innovation and Universities (MCIU). We are grateful to Sowgata Chowdhury, supported by Polish NCN grant 2015/18/A/ST9/00578, for conducting some spectroscopic observations that are reported in this work. This paper includes data collected by the TESS mission, which are publicly available from the Mikulski Archive for Space Telescopes (MAST). Funding for the TESS mission is provided by NASA's Science Mission directorate. Funding for the TESS Asteroseismic Science Operations Centre is provided by the Danish National Research Foundation (Grant agreement no.: DNRF106), ESA PRODEX (PEA 4000119301) and Stellar Astrophysics Centre (SAC) at Aarhus University. We thank the TESS team and staff and TASC/TASOC for their support of this work. This research has made use of the SIMBAD data base, operated at CDS, Strasbourg, France. This work has made use of data from the European Space Agency (ESA) mission Gaia (https://www.cosmos.esa.int/gaia), processed by the Gaia Data Processing and Analysis Consortium (DPAC, https: //www.cosmos.esa.int/web/gaia/dpac/consortium). Funding for the DPAC has been provided by national institutions, in particular the institutions participating in the Gaia Multilateral Agreement.

\section{DATA AVAILABILITY}

Data used in this work are available upon reasonable request from the corresponding author.

\section{REFERENCES}

Abt H. A., Morrell N. I., 1995, ApJS, 99, 135

Abt H. A., Brodzik D., Schaefer B., 1979, PASP, 91, 176

Alecian G., Stift M. J., 2010, A\&A, 516, A53

Alentiev D., Kochukhov O., Ryabchikova T., Cunha M., Tsymbal V., Weiss W., 2012, MNRAS, 421, L82

Ammler-von Eiff M., Reiners A., 2012, A\&A, 542, A116

Ammons S. M., Robinson S. E., Strader J., Laughlin G., Fischer D., Wolf A., 2006, ApJ, 638, 1004

Anders F. et al., 2019, A\&A, 628, A94

Avelino P. P., Cunha M. S., Chaplin W. J., 2020, MNRAS, 492, 4477

Babcock H. W., 1960, ApJ, 132, 521

Bagnulo S., Fossati L., Landstreet J. D., Izzo C., 2015, A\&A, 583, A115

Bai Y., Liu J., Bai Z., Wang S., Fan D., 2019, AJ, 158, 93

Baldry I. K., Bedding T. R., Viskum M., Kjeldsen H., Frandsen S., 1998a, MNRAS, 295, 33

Baldry I. K., Kurtz D. W., Bedding T. R., 1998b, MNRAS, 300, L39

Baldry I. K., Viskum M., Bedding T. R., Kjeldsen H., Frandsen S., 1999, MNRAS, 302, 381

Balmforth N. J., Cunha M. S., Dolez N., Gough D. O., Vauclair S., 2001, MNRAS, 323, 362

Balona L. A. et al., 2011, MNRAS, 413, 2651

Balona L. A., Laney C. D., 2003, MNRAS, 344, 242

Balona L. A., Holdsworth D. L., Cunha M. S., 2019, MNRAS, 487, 2117

Bernhard K., Hümmerich S., Paunzen E., 2020, MNRAS, 493, 3293

Bidelman W. P., 1981, AJ, 86, 553 
Bidelman W. P., MacConnell D. J., 1973, AJ, 78, 687

Bigot L., Dziembowski W. A., 2002, A\&A, 391, 235

Bigot L., Kurtz D. W., 2011, A\&A, 536, A73

Binnenfeld A., Shahaf S., Zucker S., 2020, A\&A, 642, A146

Blanco-Cuaresma S., 2019, MNRAS, 486, 2075

Blanco-Cuaresma S., Soubiran C., Heiter U., Jofré P., 2014, A\&A, 569, A111

Bonsack W. K., 1974, PASP, 86, 408

Bowman D. M., Kurtz D. W., 2018, MNRAS, 476, 3169

Bramall D. G. et al., 2010, in McLean I. S., Ramsay S. K., Takami H., eds, Proc. SPIE Conf. Ser. Vol. 7735, Ground-based and Airborne Instrumentation for Astronomy III. SPIE, Bellingham, p. 77354F

Bruntt H. et al., 2008, MNRAS, 386, 2039

Bruntt H. et al., 2009, MNRAS, 396, 1189

Buckley D. A. H., Swart G. P., Meiring J. G., 2006, in Stepp L. M., ed., Proc. SPIE Conf. Ser. Vol. 6267, Ground-based and Airborne Telescopes. SPIE, Bellingham, p. $62670 \mathrm{Z}$

Buscombe W., Foster B. E., 1995, MK spectral classifications. Twelfth general catalogue, Epoch 2000 including UBV photometry, Northwestern University, Evanston, Illinois

Butler R. P. et al., 2017, AJ, 153, 208

Bychkov V. D., Bychkova L. V., Madej J., 2009, MNRAS, 394, 1338

Cannon A. J., Pickering E. C., 1924, Annals of Harvard College Observatory, 99,1

Chandler C. O., McDonald I., Kane S. R., 2016, AJ, 151, 59

Chen P. S., Liu J. Y., Shan H. G., 2017, AJ, 153, 218

Chojnowski S. D. et al., 2019, ApJ, 873, L5

Corbally C. J., 1984, ApJS, 55, 657

Crause L. A. et al., 2014, in Ramsay S. K., McLean I. S., Takami H., eds, Proc. SPIE Conf. Ser. Vol. 9147, Ground-based and Airborne Instrumentation for Astronomy V. SPIE, Bellingham, p. 91476T

Crause L. A. et al., 2016, in Evans C. J., Simard L., Takami H., eds, Proc. SPIE Conf. Ser. Vol. 9908, Ground-based and Airborne Instrumentation for Astronomy VI. SPIE, Bellingham, p. 990827

Crawford D. L., 1979, AJ, 84, 1858

Cunha M. S., 2001, MNRAS, 325, 373

Cunha M. S., 2002, MNRAS, 333, 47

Cunha M. S., 2006, MNRAS, 365, 153

Cunha M. S., Gough D., 2000, MNRAS, 319, 1020

Cunha M. S., Fernandes J. M. M. B., Monteiro M. J. P. F. G., 2003, MNRAS, 343,831

Cunha M. S., Alentiev D., Brandão I. M., Perraut K., 2013, MNRAS, 436, 1639

Cunha M. S. et al., 2019, MNRAS, 487, 3523

Cunha M. S., Avelino P. P., Chaplin W. J., 2020, MNRAS, 499, 4687

David-Uraz A. et al., 2019, MNRAS, 487, 304

Deal M., Cunha M. S., Keszthelyi Z., Perraut K., Holdsworth D. L., 2021, preprint (arXiv:2104.08097)

Desidera S. et al., 2015, A\&A, 573, A126

Dorokhova T. N., Dorokhov N. I., 1998, Contrib. Astron. Obs. Skalnate Pleso, 27,338

Elkin V., Kurtz D. W., Mathys G., 2008, Contrib. Astron. Obs. Skalnate Pleso, 38, 317

Elkin V. G., Riley J. D., Cunha M. S., Kurtz D. W., Mathys G., 2005a, MNRAS, 358, 665

Elkin V. G., Kurtz D. W., Mathys G., 2005b, MNRAS, 364, 864

Elkin V. G., Kurtz D. W., Mathys G., Freyhammer L. M., 2010, MNRAS, 404, L104

Elkin V. G., Kurtz D. W., Worters H. L., Mathys G., Smalley B., van Wyk F., Smith A. M. S., 2011, MNRAS, 411, 978

Elkin V. G., Kurtz D. W., Nitschelm C., 2012, MNRAS, 420, 2727

Freyhammer L. M., Kurtz D. W., Cunha M. S., Mathys G., Elkin V. G., Riley J. D., 2008a, MNRAS, 385, 1402

Freyhammer L. M., Elkin V. G., Kurtz D. W., Mathys G., Martinez P., 2008b, MNRAS, 389, 441

Freyhammer L. M., Kurtz D. W., Elkin V. G., Mathys G., Savanov I., Zima W., Shibahashi H., Sekiguchi K., 2009, MNRAS, 396, 325

Gaia Collaboration, 2016, A\&A, 595, A1
Gaia Collaboration, 2018, A\&A, 616, A1

Gaia Collaboration, 2021, A\&A, 649, A1

Gelbmann M., Kupka F., Weiss W. W., Mathys G., 1997, A\&A, 319, 630

Gelbmann M., Ryabchikova T., Weiss W. W., Piskunov N., Kupka F., Mathys G., 2000, A\&A, 356, 200

Ghazaryan S., Alecian G., Hakobyan A. A., 2019, MNRAS, 487, 5922

Gilmore G. et al., 2012, Messenger, 147, 25

Glagolevskij Y. V., 1994, Bull. Special Astrophys. Obs., 38, 152

Gontcharov G. A., 2006, Astron. Lett., 32, 759

González J. F., Hubrig S., Kurtz D. W., Elkin V., Savanov I., 2008, MNRAS, 384,1140

Gray R. O., Corbally Christopher J., 2009, Stellar Spectral Classification. Princeton Univ. Press, Princeton

Gray R. O., Corbally C. J., 1994, AJ, 107, 742

Gray R. O., Corbally C. J., Garrison R. F., McFadden M. T., Bubar E. J., McGahee C. E., O’Donoghue A. A., Knox E. R., 2006, AJ, 132, 161

Grenier S. et al., 1999, A\&AS, 137, 451

Gruberbauer M. et al., 2008, A\&A, 480, 223

Gruberbauer M. et al., 2011, A\&A, 530, A135

Gustafsson B., Edvardsson B., Eriksson K., Jørgensen U. G., Nordlund, Å., Plez B., 2008, A\&A, 486, 951

Haakonsen C. B., Rutledge R. E., 2009, ApJS, 184, 138

Handler G., Paunzen E., 1999, A\&AS, 135, 57

Handler G. et al., 2006, MNRAS, 366, 257

Hartmann M., Hatzes A. P., 2015, A\&A, 582, A84

Hauck B., Mermilliod M., 1998, A\&AS, 129, 431

Hearnshaw J. B., Barnes S. I., Frost N., Kershaw G. M., Graham G., Nankivell G. R., 2003, in Ikeuchi S., Hearnshaw J., Hanawa T., eds, ASP Conf. Ser. Vol. 289, The Proceedings of the IAU 8th Asian-Pacific Regional Meeting. Astron. Soc. Pac., San Francisco, p. 11

Hensberge H. et al., 1981, A\&AS, 46, 151

Hey D. R. et al., 2019, MNRAS, 488, 18

Holdsworth D. L., 2016, Inf. Bull. Var. Stars, 6185, 1

Holdsworth D. L., 2021, Front. Astron. Space Sci., 8, 31

Holdsworth D. L. et al., 2014a, MNRAS, 439, 2078

Holdsworth D. L., Smalley B., Kurtz D. W., Southworth J., Cunha M. S., Clubb K. I., 2014b, MNRAS, 443, 2049

Holdsworth D. L., Kurtz D. W., Smalley B., Saio H., Handler G., Murphy S. J., Lehmann H., 2016, MNRAS, 462, 876

Holdsworth D. L. et al., 2018a, MNRAS, 473, 91

Holdsworth D. L., Saio H., Bowman D. M., Kurtz D. W., Sefako R. R., Joyce M., Lambert T., Smalley B., 2018b, MNRAS, 476, 601

Holdsworth D. L., Cunha M. S., Shibahashi H., Kurtz D. W., Bowman D. M., 2018c, MNRAS, 480, 2976

Holdsworth D. L., Saio H., Kurtz D. W., 2019, MNRAS, 489, 4063

Houk N., 1978, Michigan Catalogue of Two-Dimensional Spectral Types for the HD Stars. Univ. Michigan, Ann Arbor

Houk N., 1982, Michigan Catalogue of Two-dimensional Spectral Types for the HD stars. Vol._3. Declinations $-40_{-} \int 0$ to $-26-\int 0$. Univ. Michigan, Ann Arbor

Houk N., Cowley A. P., 1975, University of Michigan Catalogue of twodimensional spectral types for the HD stars. Volume I. Declinations -90_

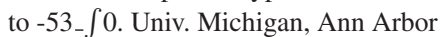

Houk N., Smith-Moore M., 1988, Michigan Catalogue of Two-dimensional Spectral Types for the HD Stars. Volume 4, Declinations -26。.0 to -12॰.0. Vol. 4. Univ. Michigan, Ann Arbor

Houk N., Swift C., 1999a, Michigan Spectral Survey 5, Univ. Michigan, Ann Arbor

Houk N., Swift C., 1999b, Michigan catalogue of two-dimensional spectral types for the HD Stars; Vol. 5. Univ. Michigan, Ann Arbor

Hubrig S., Nesvacil N., 2007, MNRAS, 378, L16

Hubrig S., Kharchenko N., Mathys G., North P., 2000, A\&A, 355, 1031

Hubrig S., Szeifert T., Schöller M., Mathys G., Kurtz D. W., 2004, A\&A, 415,685

Hubrig S. et al., 2005, A\&A, 440, L37

Hubrig S., North P., Schöller M., Mathys G., 2006, Astron. Nachr., 327, 289 
Hubrig S., Järvinen S. P., Madej J., Bychkov V. D., Ilyin I., Schöller M., Bychkova L. V., 2018, MNRAS, 477, 3791

Hümmerich S., Paunzen E., Bernhard K., 2016, AJ, 152, 104

Jackson J., Stoy R. H., 1954, Annals of the Cape Observatory, 17,258

Jenkins J. M. et al., 2016, in Chiozzi G., Guzman J. C., eds, Proc. SPIE Conf. Ser. Vol. 9913, Software and Cyberinfrastructure for Astronomy IV. SPIE, Bellingham, p. 99133E

Joshi S. et al., 2016, A\&A, 590, A116

Kervella P., Arenou F., Mignard F., Thévenin F., 2019, A\&A, 623, A72

Khalack V. et al., 2019, MNRAS, 490, 2102

Kniazev A. Y., Gvaramadze V. V., Berdnikov L. N., 2016, MNRAS, 459, 3068

Kniazev A. Y., Gvaramadze V. V., Berdnikov L. N., 2017, in Balega Y. Y., Kudryavtsev D. O., Romanyuk I. I., Yakunin I. A., eds, ASP Conf. Ser. Vol. 510, Stars: From Collapse to Collapse. Astron. Soc. Pac., San Francisco, p. 480

Kobzar O. et al., 2020, in Wade G., Alecian E., Bohlender D., Sigut A., eds, Stellar Magnetism: A Workshop in Honour of the Career and Contributions of John D. Landstreet,Vol. 11, Proceedings of the Polish Astronomical Society,, p. 214, preprint (arXiv:2003.05337)

Kobzar O., 2020, Master's thesis, Université de Moncton, Canada

Kochukhov O., 2003, A\&A, 404, 669

Kochukhov O., 2004, ApJ, 615, L149

Kochukhov O., 2006, A\&A, 446, 1051

Kochukhov O., 2011, in Prasad Choudhary D., Strassmeier K. G., eds, Physics of Sun and Star Spots, Vol. 273. Cambridge Univ. Press, Cambridge, p. 249,

Kochukhov O., Bagnulo S., 2006, A\&A, 450, 763

Kochukhov O., Ryabchikova T., 2001, A\&A, 377, L22

Kochukhov O., Drake N. A., Piskunov N., de la Reza R., 2004, A\&A, 424, 935

Kochukhov O., Ryabchikova T., Weiss W. W., Landstreet J. D., Lyashko D., 2007, MNRAS, 376, 651

Kochukhov O., Ryabchikova T., Bagnulo S., Lo Curto G., 2008, A\&A, 479, L29

Kochukhov O., Bagnulo S., Lo Curto G., Ryabchikova T., 2009a, A\&A, 493, L45

Kochukhov O., Shulyak D., Ryabchikova T., 2009b, A\&A, 499, 851

Kochukhov O., Alentiev D., Ryabchikova T., Boyko S., Cunha M., Tsymbal V., Weiss W., 2013, MNRAS, 431, 2808

Kordopatis G. et al., 2013, AJ, 146, 134

Kreidl T. J., 1990, Inf. Bull. Var. Stars, 3539, 1

Kreidl T. J., Kurtz D. W., Bus S. J., Kuschnig R., Birch P. B., Candy M. P., Weiss W. W., 1991, MNRAS, 250, 477

Kudryavtsev D. O., Romanyuk I. I., 2003, Astrophysics, 46, 234

Kudryavtsev D. O., Romanyuk I. I., 2012, Astron. Nachr., 333, 41

Kudryavtsev D. O., Romanyuk I. I., Elkin V. G., Paunzen E., 2006, MNRAS, 372, 1804

Kudryavtsev D. O., Romanyuk I. I., Semenko E. A., Solov'ev G. A., 2007, Astrophys. Bull., 62, 147

Kunder A. et al., 2017, AJ, 153, 75

Kupka F., Ryabchikova T. A., Weiss W. W., Kuschnig R., Rogl J., Mathys G., 1996, A\&A, 308, 886

Kurtz D. W., 1978, Inf. Bull. Var. Stars, 1436, 1

Kurtz D. W., 1981, Inf. Bull. Var. Stars, 1915, 1

Kurtz D. W., 1982, MNRAS, 200, 807

Kurtz D. W., 1983, MNRAS, 205, 3

Kurtz D. W., 1984, MNRAS, 209, 841

Kurtz D. W., 1985, MNRAS, 213, 773

Kurtz D. W., 1990, MNRAS, 242, 489

Kurtz D. W., Cropper M. S., 1981, Inf. Bull. Var. Stars, 1987, 1

Kurtz D. W., Holdsworth D. L., 2020, Astrophys. Space Sci. Proc., 57, 313

Kurtz D. W., Kreidl T. J., 1985, MNRAS, 216, 987

Kurtz D. W., Marang F., 1987a, MNRAS, 228, 141

Kurtz D. W., Marang F., 1987b, MNRAS, 229, 285

Kurtz D. W., Martinez P., 1987, MNRAS, 226, 187
Kurtz D. W., Martinez P., 1993, in Dworetsky M. M., Castelli F., Faraggiana R., eds, ASP. Conf. Ser. Vol. 44, IAU Colloq. 138: Peculiar versus Normal Phenomena in A-type and Related Stars. Astron. Soc. Pac., San Francisco, p. 561

Kurtz D. W., Martinez P., 1994, Inf. Bull. Var. Stars, 4013, 1

Kurtz D. W., Allen S., Cropper M. S., 1981, Inf. Bull. Var. Stars, 2033, 1

Kurtz D. W., van Wyk F., Marang F., 1990, MNRAS, 243, 289

Kurtz D. W., Sullivan D. J., Martinez P., Tripe P., 1994a, MNRAS, 270, 674

Kurtz D. W., Martinez P., Tripe P., 1994b, MNRAS, 271, 421

Kurtz D. W., Marang F., van Wyk F., Roberts G., 1996a, MNRAS, 280, 1

Kurtz D. W., Martinez P., Koen C., Sullivan D. J., 1996b, MNRAS, 281, 883

Kurtz D. W., van Wyk F., Roberts G., Marang F., Handler G., Medupe R., Kilkenny D., 1997a, MNRAS, 287, 69

Kurtz D. W., Martinez P., Tripe P., Hanbury A. G., 1997b, MNRAS, 289, 645

Kurtz D. W., Elkin V. G., Mathys G., 2003, MNRAS, 343, L5

Kurtz D. W. et al., 2005, MNRAS, 358, 651

Kurtz D. W., Elkin V. G., Mathys G., 2006a, MNRAS, 370, 1274

Kurtz D. W., Elkin V. G., Cunha M. S., Mathys G., Hubrig S., Wolff B., Savanov I., 2006b, MNRAS, 372, 286

Kurtz D. W., Hubrig S., González J. F., van Wyk F., Martinez P., 2008, MNRAS, 386, 1750

Kurtz D. W. et al., 2011, MNRAS, 414, 2550

Landstreet J. D., Mathys G., 2000, A\&A, 359, 213

LeBlanc F., Monin D., Hui-Bon-Hoa A., Hauschildt P. H., 2009, A\&A, 495, 937

Lenz P., Breger M., 2005, Commun. Asteroseismol., 146, 53

Lorenz D., Handler G., Kurtz D. W., 2005, Inf. Bull. Var. Stars, 5651, 1

Lüftinger T., Kochukhov O., Ryabchikova T., Piskunov N., Weiss W. W., Ilyin I., 2010, A\&A, 509, A71

Manfroid J., Mathys G., 1986, A\&AS, 64, 9

Martinez P., 1991, Inf. Bull. Var. Stars, 3621, 1

Martinez P., Kauffmann G., 1990, Inf. Bull. Var. Stars, 3507, 1

Martinez P., Kurtz D. W., 1990, Inf. Bull. Var. Stars, 3509, 1

Martinez P., Kurtz D. W., 1991a, Inf. Bull. Var. Stars, 3553, 1

Martinez P., Kurtz D. W., 1991b, Inf. Bull. Var. Stars, 3611, 1

Martinez P., Kurtz D. W., 1992, Inf. Bull. Var. Stars, 3750, 1

Martinez P., Kurtz D. W., 1994a, MNRAS, 271, 118

Martinez P., Kurtz D. W., 1994b, MNRAS, 271, 129

Martinez P., Kurtz D. W., Heller C. H., 1990, MNRAS, 246, 699

Martinez P., Kurtz D. W., Kauffmann G. M., 1991, MNRAS, 250, 666

Martinez P., Kurtz D. W., Kreidl T. J., Koen C., van Wyk F., Marang F., Roberts G., 1993a, MNRAS, 263, 273

Martinez P., Kurtz D. W., Ashley R., 1993b, Inf. Bull. Var. Stars, 3844, 1

Martinez P., Kurtz D. W., van Wyk F., 1994, MNRAS, 271, 305

Martinez P., Kurtz D. W., Hoffman M. J. H., van Wyk F., 1995, MNRAS, 276, 1435

Martinez P., Marang F., van Wyk F., Roberts G. R., 1998a, Observatory, 118, 153

Martinez P., Koen C., Sullivan D. J., 1998b, MNRAS, 300, 188

Martinez P., Meintjes P., Ratcliff S. J., Engelbrecht C., 1998c, A\&A, 334, 606

Masana E., Jordi C., Maitzen H. M., Torra J., 1998, A\&AS, 128, 265

Mason B. D., Wycoff G. L., Hartkopf W. I., Douglass G. G., Worley C. E., 2001, AJ, 122, 3466

Mathys G., 1985, A\&A, 151, 315

Mathys G., 1991, A\&AS, 89, 121

Mathys G., 2017, A\&A, 601, A14

Mathys G., Hubrig S., 1997, A\&AS, 124, 475

Mathys G., Kharchenko N., Hubrig S., 1996, A\&A, 311, 901

Mathys G., Hubrig S., Landstreet J. D., Lanz T., Manfroid J., 1997, A\&AS, 123,353

Mathys G., Kurtz D. W., Elkin V. G., 2007, MNRAS, 380, 181

Mathys G., Khalack V., Landstreet J. D., 2020a, A\&A, 636, A6

Mathys G., Kurtz D. W., Holdsworth D. L., 2020b, A\&A, 639, A31

Matthews J. M., 1987, PhD thesis, The University of Western Ontario, Canada

Matthews J. M., Kurtz D. W., Martinez P., 1999, ApJ, 511, 422

McDonald I., Zijlstra A. A., Boyer M. L., 2012, MNRAS, 427, 343

McDonald I., Zijlstra A. A., Watson R. A., 2017, MNRAS, 471, 770 
Medupe R., Kurtz D. W., Elkin V. G., Mguda Z., Mathys G., 2015, MNRAS, 446, 1347

Michaud G., Megessier C., Charland Y., 1981, A\&A, 103, 244

Mkrtichian D. E., Hatzes A. P., 2005, A\&A, 430, 263

Moon T. T., Dworetsky M. M., 1985, MNRAS, 217, 305

Murphy S. J., Hey D., Van Reeth T., Bedding T. R., 2019, MNRAS, 485, 2380

Murphy S. J., Saio H., Takada-Hidai M., Kurtz D. W., Shibahashi H., Takata M., Hey D. R., 2020, MNRAS, 498, 4272

Nelson M. J., Kreidl T. J., 1993, AJ, 105, 1903

Netopil M., Paunzen E., Hümmerich S., Bernhard K., 2017, MNRAS, 468, 2745

North P., 1984, A\&A, 141, 328

North P., Jaschek C., Hauck B., Figueras F., Torra J., Kunzli M., 1997, in Bonnet R. M. , et al., eds, ESA Special Publication Vol. 402, Hipparcos Venice '97. ESA Publications Division,Noordwijk, p. 239

Oelkers R. J. et al., 2018, AJ, 155, 39

Paunzen E. et al., 2018, Res. Astron. Astrophys., 18, 135

Paunzen E., 2015, A\&A, 580, A23

Paunzen E., Netopil M., Rode-Paunzen M., Handler G., Božić H., Ruždjak D., Sudar D., 2012, A\&A, 542, A89

Perraut K., Brandão I., Cunha M., Shulyak D., Mourard D., Nardetto N., ten Brummelaar T. A., 2016, A\&A, 590, A117

Perry C. L., 1991, PASP, 103, 494

Perry C. L., Christodoulou D. M., 1996, PASP, 108, 772

Pollacco D. L. et al., 2006, PASP, 118, 1407

Quitral-Manosalva P., Cunha M. S., Kochukhov O., 2018, MNRAS, 480, 1676

Reiners A., Zechmeister M., 2020, ApJS, 247, 11

Renson P., Manfroid J., 2009, A\&A, 498, 961

Renson P., Gerbaldi M., Catalano F. A., 1991, A\&AS, 89, 429

Ricker G. R. et al., 2015, J. Astron. Telesc. Instrum. Syst., 1, 014003

Romanyuk I. I., Semenko E. A., Kudryavtsev D. O., Moiseevaa A. V., 2016, Astrophys. Bull., 71, 302

Rusomarov N. et al., 2013, A\&A, 558, A8

Rusomarov N., Kochukhov O., Ryabchikova T., Piskunov N., 2015, A\&A, 573, A123

Ryabchikova T., Nesvacil N., Weiss W. W., Kochukhov O., Stütz C., 2004, A\&A, 423, 705

Ryabchikova T., Sachkov M., Kochukhov O., Lyashko D., 2007, A\&A, 473, 907

Ryabchikova T., Kochukhov O., Bagnulo S., 2008, A\&A, 480, 811

Ryabchikova T., Piskunov N., Kurucz R. L., Stempels H. C., Heiter U., Pakhomov Y., Barklem P. S., 2015, Phys. Scr, 90, 054005

Sahoo S. K., Baran A. S., Sanjayan S., Ostrowski J., 2020, MNRAS, 499, 5508

Saio H., 2005, MNRAS, 360, 1022

Saio H., Gautschy A., 2004, MNRAS, 350, 485

Samus' N. N. et al., 2003, Astron. Lett., 29, 468

Samus' N. N., Kazarovets E. V., Durlevich O. V., Kireeva N. N., Pastukhova E. N., 2017, Astron. Rep., 61, 80

Schneider H., Weiss W. W., 1990, Inf. Bull. Var. Stars, 3520, 1

Schneider H., Kreidl T. J., Weiss W. W., 1992, A\&A, 257, 130

Schofield M. et al., 2019, ApJS, 241, 12

Shavrina A. V., Khalack V., Glagolevskij Y., Lyashko D., Landstreet J., Leone F., Giarrusso M., 2014, in Petit P., Jardine M., Spruit H. C., eds, Magnetic Fields throughout Stellar Evolution, Vol. 302. Cambridge Univ. Press, Cambrige, p. 274

Shibahashi H., 1979, PASJ, 31, 87

Shibahashi H., Saio H., 1985, PASJ, 37, 601

Sikora J., Wade G. A., Power J., Neiner C., 2019a, MNRAS, 483, 2300

Sikora J., Wade G. A., Power J., Neiner C., 2019b, MNRAS, 483, 3127

Sikora J. et al., 2019c, MNRAS, 487, 4695

Skiff B. A., 2014, VizieR Online Data Catalog, p. B/mk

Smalley B. et al., 2015, MNRAS, 452, 3334

Sousa J. C., Cunha M. S., 2011, MNRAS, 414, 2576

Stassun K. G. et al., 2019, AJ, 158, 138

Stevens D. J., Stassun K. G., Gaudi B. S., 2017, AJ, 154, 259
Stibbs D. W. N., 1950, MNRAS, 110, 395

Stępień K., 2000, A\&A, 353, 227

Suchkov A. A., Makarov V. V., Voges W., 2003, ApJ, 595, 1206

Tassoul M., 1980, ApJS, 43, 469

Trifonov T., Tal-Or L., Zechmeister M., Kaminski A., Zucker S., Mazeh T., 2020, A\&A, 636, A74

Vauclair S., Théado S., 2004, A\&A, 425, 179

Vogt N., Faundez A. M., 1979, A\&AS, 36, 477

Wade G. A., 1997, A\&A, 325, 1063

Watson C. L., Henden A. A., Price A., 2006, Soc. Astron. Sci. Annu. Symp., 25,47

Weiss W. W. et al., 2016, A\&A, 588, A54

Weiss W. W. et al., 2020, A\&A, 642, A64

White T. R., Bedding T. R., Stello D., Kurtz D. W., Cunha M. S., Gough D. O., 2011, MNRAS, 415, 1638

\section{APPENDIX A: SPECTRAL CLASSIFICATION PLOTS}

Here we provide the spectral classification plots used to provide new or updated spectral classifications. We have used the SpUpNIC long slit spectrograph mounted on the SAAO 1.9-m telescope to

Table A1. Log of the spectroscopic observations collected for stars presented in this paper. The resolution refers to the canonical resolving power of the instrument in the configuration used. The final column shows the number of observations per instrument, with a ' $\mathrm{B}$ ' or ' $\mathrm{R}$ ' referring to the $\# 4$ or $\# 6$ grating of the SpUpNIC instrument. For full details see Section 3. The table is split into three sections to reflect Table 1, but with the known roAp stars prior to TESS launch section excluded.

\begin{tabular}{|c|c|c|c|}
\hline TIC & Spectrograph & Resolution & Nobs \\
\hline 119327278 & HRS & 63000 & 1 \\
\hline \multirow[t]{2}{*}{170586794} & SpUpNIC & 7500 & 1B \\
\hline & HRS & 63000 & 1 \\
\hline 176516923 & SpUpNIC & 7500 & $1 \mathrm{~B}$ \\
\hline 294266638 & SpUpNIC & 7500 & 1B \\
\hline 310817678 & HRS & 43000 & 4 \\
\hline 356088697 & SpUpNIC & 7500 & 1B \\
\hline 380651050 & HRS & 63000 & 1 \\
\hline 466260580 & HRS & 63000 & 1 \\
\hline \multirow[t]{2}{*}{12968953} & SpUpNIC & 7500 & 1B \\
\hline & HRS & 63000 & 2 \\
\hline 17676722 & SpUpNIC & $7500 / 4700$ & $2 \mathrm{~B}, 1 \mathrm{R}$ \\
\hline \multirow[t]{2}{*}{41259805} & SpUpNIC & 7500 & $4 \mathrm{~B}$ \\
\hline & HRS & 63000 & 1 \\
\hline \multirow[t]{2}{*}{49818005} & SpUpNIC & 7500 & $3 \mathrm{~B}$ \\
\hline & HRS & 63000 & 1 \\
\hline \multirow[t]{2}{*}{152808505} & SpUpNIC & 7500 & 1B \\
\hline & HRS & 63000 & 1 \\
\hline \multirow[t]{2}{*}{156886111} & SpUpNIC & $7500 / 4700$ & $1 \mathrm{~B}, 1 \mathrm{R}$ \\
\hline & HRS & 63000 & 1 \\
\hline \multirow[t]{2}{*}{259587315} & SpUpNIC & 7500 & $3 \mathrm{~B}$ \\
\hline & HRS & 63000 & 1 \\
\hline \multirow[t]{2}{*}{349945078} & SpUpNIC & $7500 / 4700$ & $2 \mathrm{~B}, 3 \mathrm{R}$ \\
\hline & HRS & 63000 & 1 \\
\hline \multirow[t]{3}{*}{350146296} & SpUpNIC & $7500 / 4700$ & $5 \mathrm{~B}, 1 \mathrm{R}$ \\
\hline & HRS & 63000 & 2 \\
\hline & HERCULES & 41000 & 6 \\
\hline \multirow[t]{2}{*}{431380369} & SpUpNIC & 7500 & $2 \mathrm{~B}$ \\
\hline & HRS & 63000 & 1 \\
\hline 3814749 & SpUpNIC & 7500 & $1 \mathrm{~B}$ \\
\hline \multirow[t]{2}{*}{410163387} & SpUpNIC & 7500 & 1B \\
\hline & HRS & 63000 & 1 \\
\hline
\end{tabular}




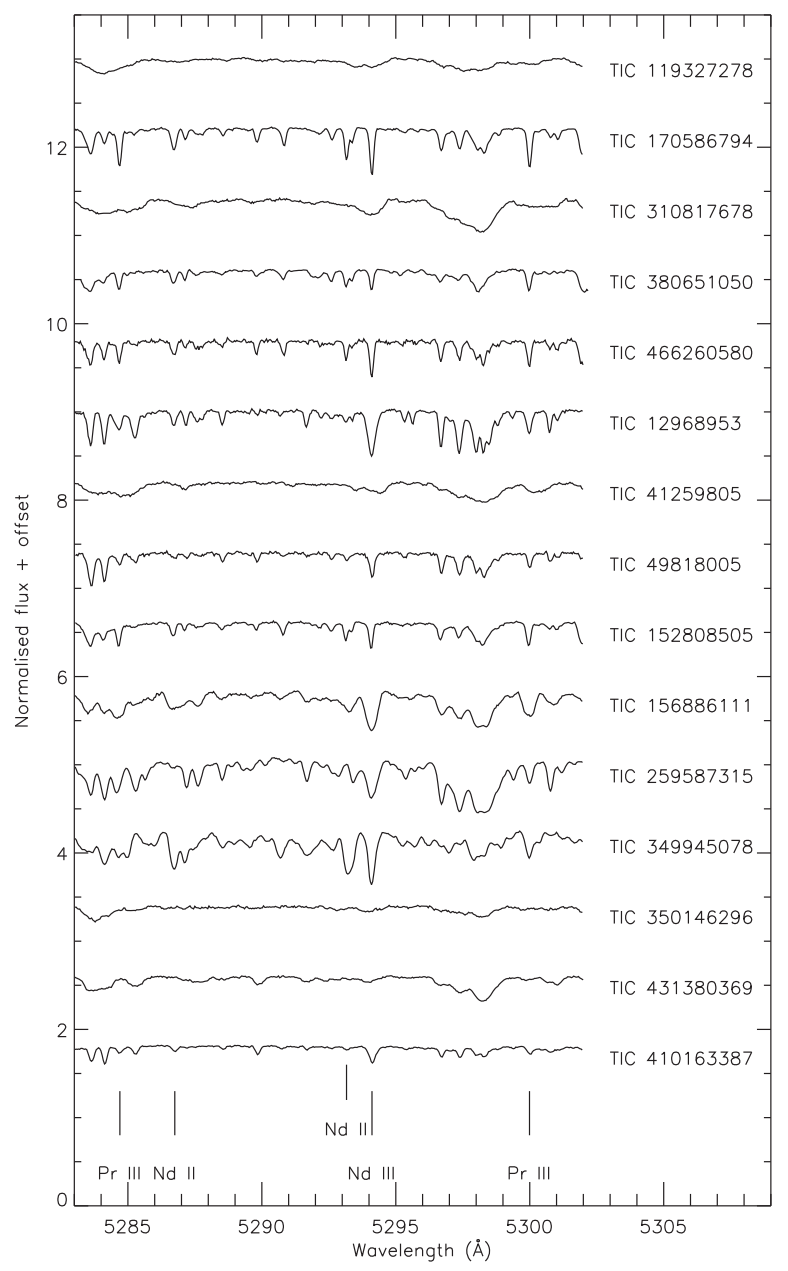

Figure A1. SALT/HRS spectra of new and candidate roAp stars, as listed in Table A1, showing a region in the blue part of the spectrum of use in identifying chemical peculiarities.

record these spectra. We used grating 4 with a grating angle of $4.2^{\circ}$, with observations typically made with a 1 arcsec slit to reach a resolving power of $R \sim 3000$, but was widened in poor seeing or low sky transparency situations. The data were flat-field and bias corrected, cleaned of cosmic rays, and had a wavelength solution applied using a CuAr reference spectrum obtained directly after the science observation. The spectra were normalized using an automated spline fitting procedure in iSPEC spectral analysis software (Blanco-Cuaresma et al. 2014; Blanco-Cuaresma 2019). We also provide, in Table A1, a log of all spectroscopic observations related to this work.

Where we have high resolution data available, we plot two regions of useful in identifying chemical peculiarities in Ap stars in Fig. A1 and Fig. A2.

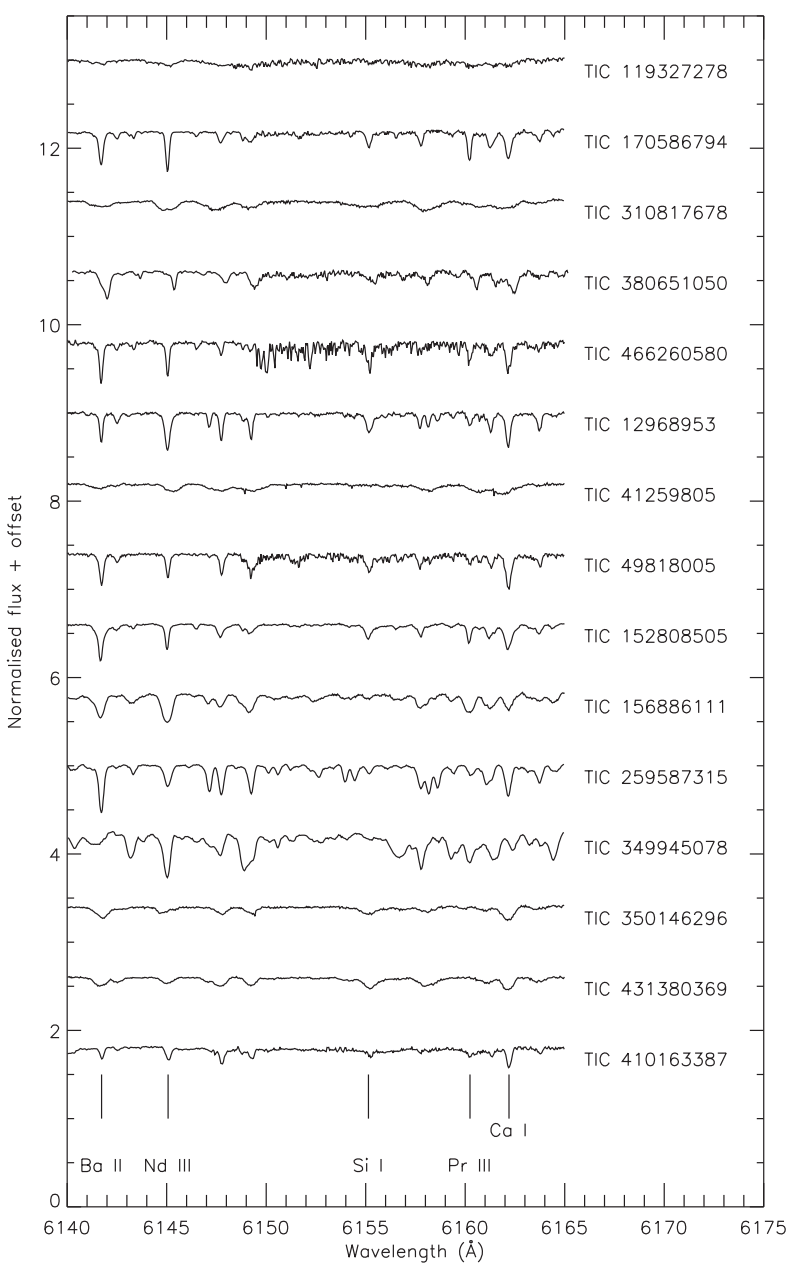

Figure A2. SALT/HRS spectra of new and candidate roAp stars, as listed in Table A1, showing a region in the red part of the spectrum of use in identifying chemical peculiarities.

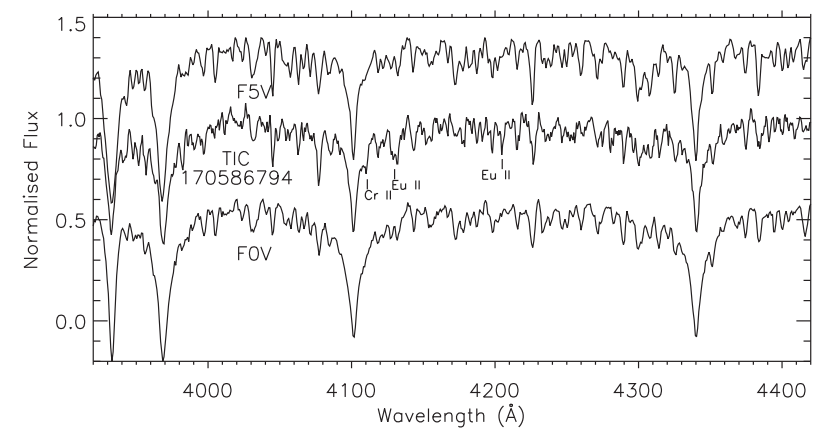

Figure A3. SAAO spectrum of TIC 170586794 (centre), with stars of spectral class F5V (above) and F0V (below) for comparison. Lines of Eu and $\mathrm{Cr}$ are enhanced in this star, leading to the classification of F5p EuCr. 


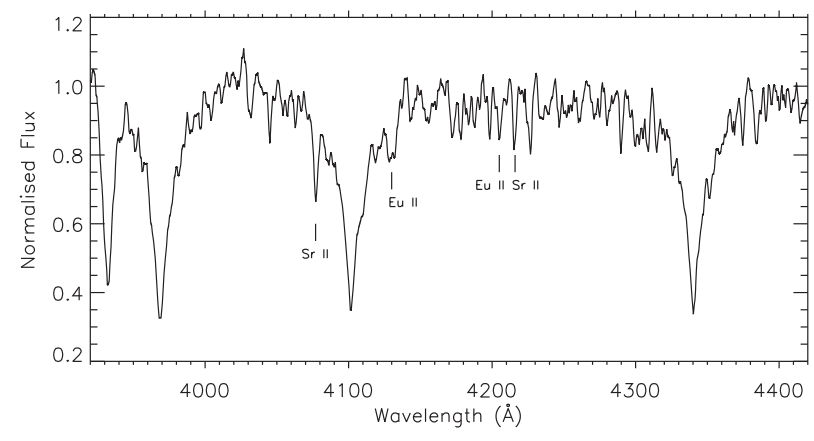

Figure A4. SAAO spectrum of TIC 294266638. Lines of Sr and Eu are noted as being pronounced in this star, leading to the classification of A7p SrEu.

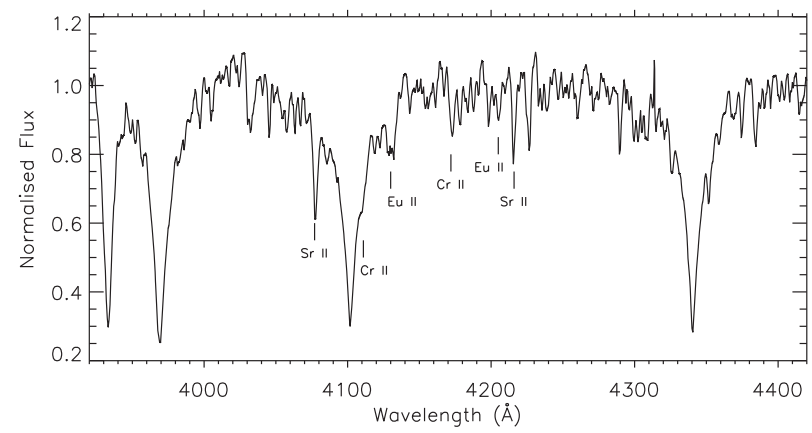

Figure A5. The SAAO spectrum of TIC 356088697 with lines of Sr, Eu, and $\mathrm{Cr}$ identified. We refine the spectral classification to A8p SrEuCr.

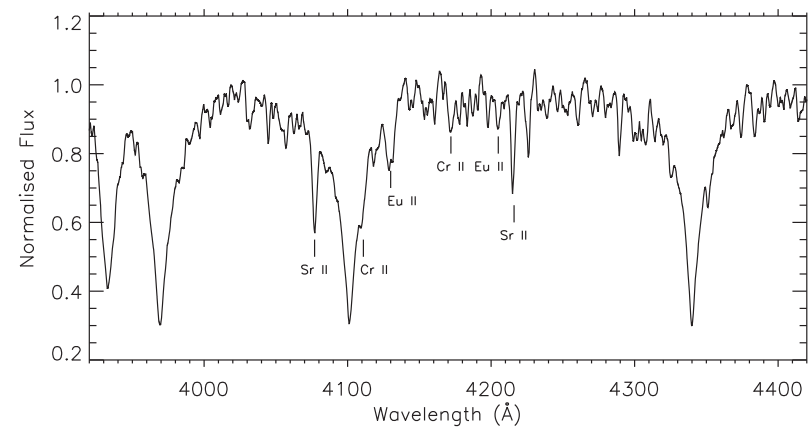

Figure A6. The SAAO spectrum of TIC 12968953 with lines of Sr, Eu, and $\mathrm{Cr}$ identified. We refine the spectral classification to $\mathrm{A} 7 \mathrm{p} \mathrm{SrEuCr}$.

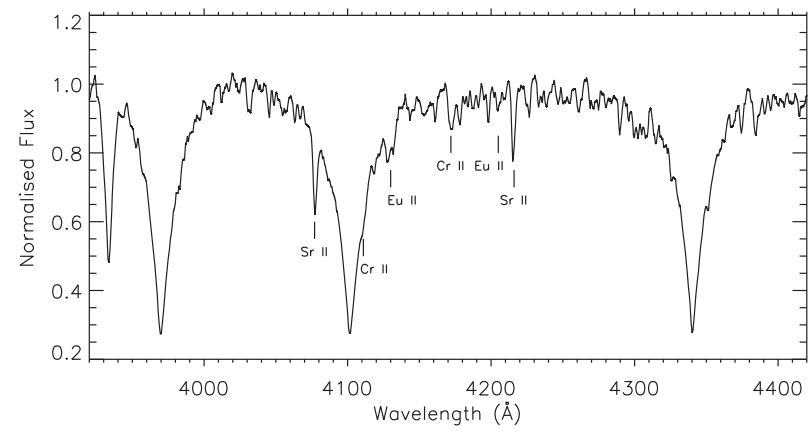

Figure A7. The SAAO spectrum of TIC 17676722 with lines of Sr, Eu, and $\mathrm{Cr}$ identified. We refine the spectral classification to $\mathrm{A} 3 \mathrm{p} \mathrm{SrEuCr}$.

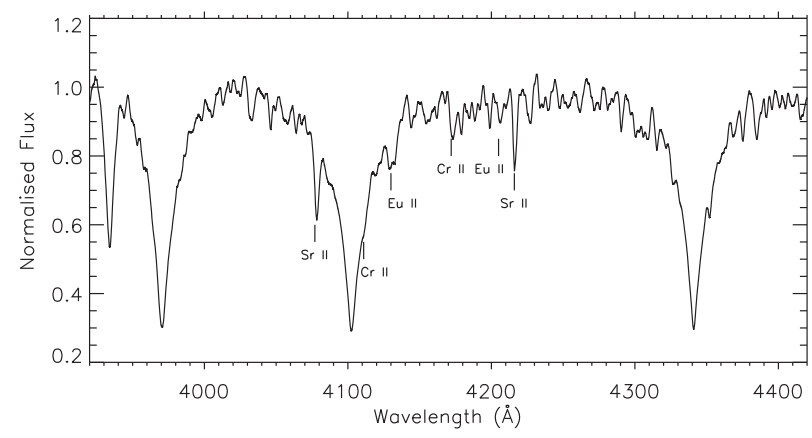

Figure A8. The SAAO spectrum of TIC 41259805 with lines of Sr, Eu, and $\mathrm{Cr}$ identified. We refine the spectral classification to $\mathrm{A} 6 \mathrm{p} \mathrm{SrEu}(\mathrm{Cr})$.

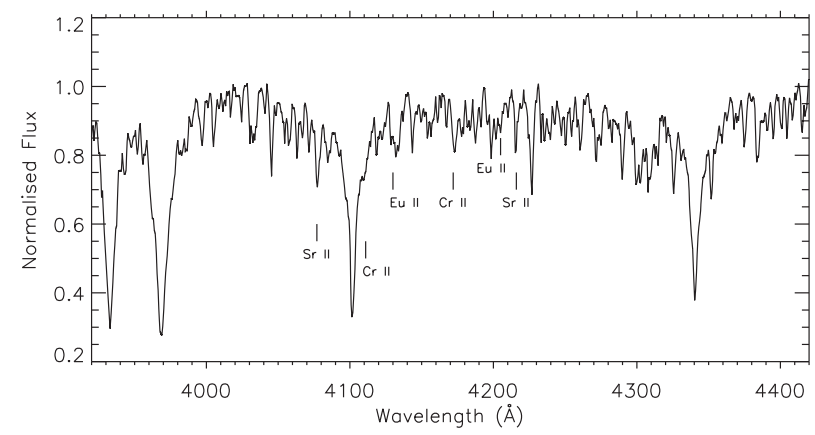

Figure A9. The SAAO spectrum of TIC 49818005 with lines of Sr, Eu, and $\mathrm{Cr}$ identified. We provide a spectral classification of $\mathrm{F} 2 \mathrm{p} \mathrm{SrEu}(\mathrm{Cr})$.

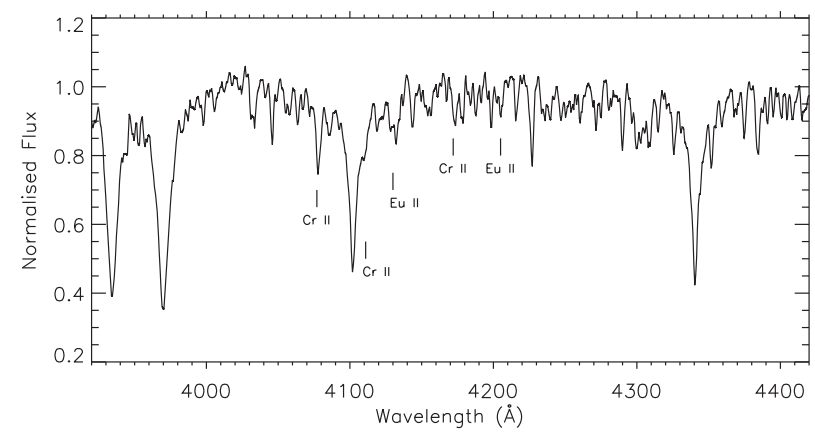

Figure A10. The SAAO spectrum of TIC 152808505 . We provide a spectral classification of F3p EuCr.

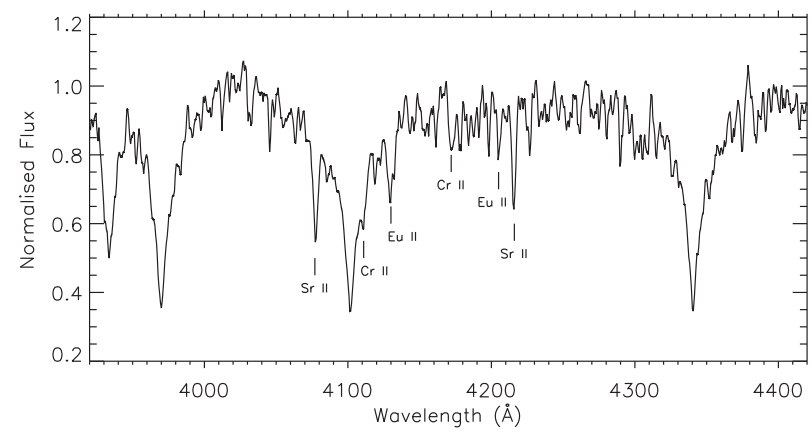

Figure A11. The SAAO spectrum of TIC 156886111, from which we deduce a classification of A8p SrEuCr. 


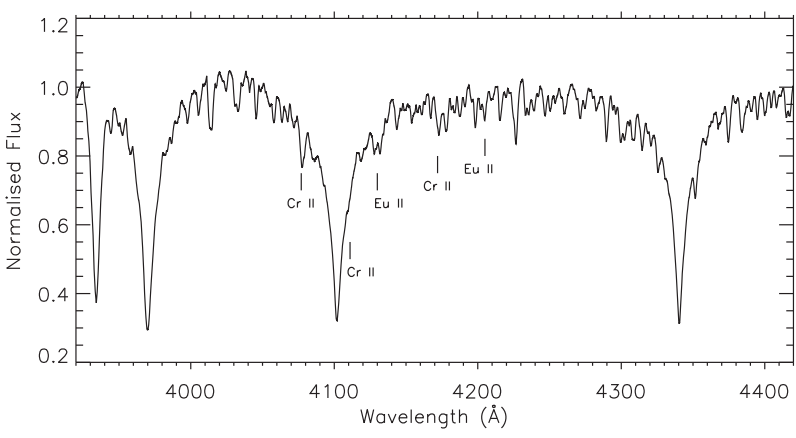

Figure A12. The SAAO spectrum of TIC 350146296, from which we deduce a classification of $\mathrm{F} 0 \mathrm{p} \mathrm{EuCr}$.

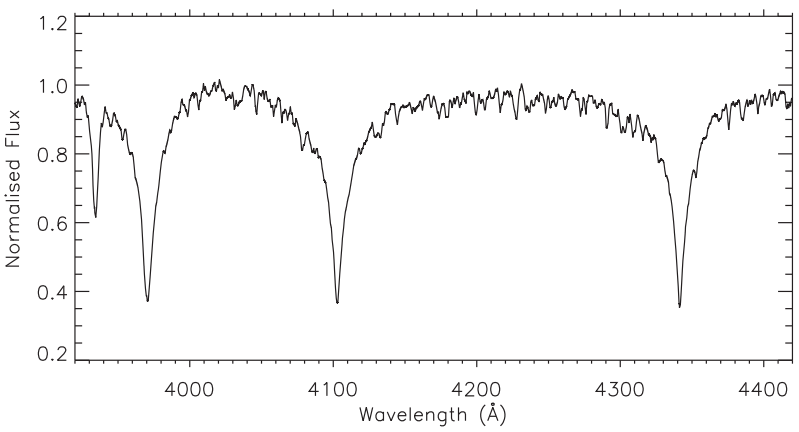

Figure A13. The SAAO spectrum of TIC 3814749 showing the lack of strong chemical peculiarities in this star.

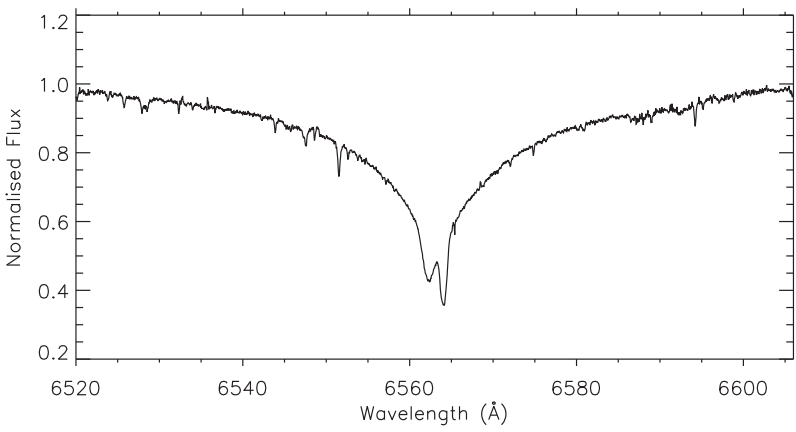

Figure A14. The SALT/HRS spectrum of the $\mathrm{H}_{\alpha}$ line in TIC 410163387 showing the star to be a spectroscopic binary system.
${ }^{1}$ Jeremiah Horrocks Institute, University of Central Lancashire, Preston PRI $2 H E, U K$

${ }^{2}$ Instituto de Astrofísica e Ciências do Espaço, Universidade do Porto CAUP, Rua das Estrelas, P-PT4150-762 Porto, Portugal

${ }^{3}$ Centre for Space Research, Physics Department, North West University, Mahikeng 2745, South Africa

${ }^{4}$ DTU Space, National Space Institute, Technical University of Denmark, Elektrovej 328, DK-2800 Kgs. Lyngby, Denmark

${ }^{5}$ Stellar Astrophysics Centre, Aarhus University, DK-8000 Aarhus C, Denmark

${ }^{6}$ School of Physics, Sydney Institute for Astronomy (SIfA), The University of Sydney, NSW 2006, Australia

${ }^{7}$ Institute of Astronomy, KU Leuven, Celestijnenlaan 200D, B-3001 Leuven, Belgium

${ }^{8}$ Département de Physique et d'Astronomie, Université de Moncton, 18 avenue Antonine-Maillet, Moncton, NB E1A 3E9, Canada

${ }^{9}$ Department of Chemistry and Physics, Florida Gulf Coast University, 10501 FGCU Blvd. S., Fort Myers, FL 33965 USA

${ }^{10}$ Department of Physics and Astronomy, Uppsala University, Box 516, SE75120 Uppsala, Sweden

${ }^{11}$ Astronomical Institute of University of Wroctaw, ul. Kopernika 11, PL-51622 Wroctaw, Poland

${ }^{12}$ Ankara University, Faculty of Science, Department of Astronomy and Space Sciences, 06100 Tandogan - Ankara / Turkey

${ }^{13}$ Department of Astronomy, School of Physics, Peking University, Beijing 100871, People's Republic of China

${ }^{14}$ Kavli Institute for Astronomy and Astrophysics, Peking University, Beijing 100871, People's Republic of China

${ }^{15}$ Konkoly Observatory, Research Centre for Astronomy and Earth Sciences, ELKH, H-1121 Konkoly Thege Miklós út 15-17, Budapest, Hungary

${ }^{16}$ MTA CSFK Lendület Near-Field Cosmology Research Group , Konkoly Thege Miklós út 15-17 1121 Budapest Hungary

${ }^{17}$ ELTE Eötvös Loránd University, Institute of Physics, Pázmány Péter sétány 1/A, H-1117 Budapest, Hungary

${ }^{18}$ Max-Planck-Institut für Sonnensystemforschung, Justus-von-Liebig-Weg 3, D-37077 Göttingen, Germany

${ }^{19}$ Núcleo de Astronomía, Facultad de Ingeniería y Ciencias, Universidad Diego Portales, Av. Ejército Libertador 441, Santiago, Chile

${ }^{20}$ Instituto de Astronomía-Universidad Nacional Autónoma de México, Ap. P. 877, Ensenada, BC 22860, Mexico

${ }^{21}$ Instituto de Astrofísica de Andalucía (CSIC), Glorieta de la Astronomía s/n, E-18008 Granada, Spain

${ }^{22}$ Physics Department, Mount Allison University, Sackville, NB E4L1E6, Canada

${ }^{23}$ National Astronomical Research Institute of Thailand, 260 Moo 4, T. Donkaew, A. Maerim, Chiangmai 50180, Thailand

${ }^{24}$ Department of Theoretical Physics and Astrophysics, Masaryk University, Kotlárská 2, CZ-611 37 Brno, Czech Republic

${ }^{25}$ School of Earth and Space Exploration, Arizona State University, Tempe, AZ 85281, USA

${ }^{26}$ Physics and Astronomy Department, Bishop's University, 2600 College Street, Sherbrooke, QC J1M 1Z7, Canada

${ }^{27}$ School of Physics, Xi'an Jiaotong University, 710049 Xi'an, People's Republic of China

${ }^{28}$ Department of Physics, University of York, Heslington, York YO10 5DD, $U K$

${ }^{29}$ School of Physical and Chemical Sciences, Te Kura Matū, University of Canterbury, Christchurch 8020, New Zealand

${ }^{30}$ Department of Physics and Astronomy, Howard University, Washington, DC 20059, USA

${ }^{31}$ Center for Research and Exploration in Space Science and Technology, and X-ray Astrophysics Laboratory, NASA/GSFC, Greenbelt, MD 20771, USA

${ }^{32}$ ELTE Eötvös Loránd University, Gothard Astrophysical Observatory, 9700 Szombathely, Hungary

${ }^{33}$ MTA-ELTE Exoplanet Research Group, 9700 Szombathely, Szent Imre h. u. 112, Hungary

${ }^{34}$ Dept. Física Teórica y del Cosmos, Universidad de Granada, Campus de Fuentenueva s/n, E-18071 Granada, Spain 
${ }^{35}$ Los Alamos National Laboratory, MS T-082, Los Alamos, NM 87545, USA

${ }^{36}$ Department of Astronomy, University of Cape Town, Private Bag X3, Rondebosch 7701, South Africa

${ }^{37}$ Núcleo de Astronomía de la Facultad de Ingeniería y Ciencias, Universidad Diego Portales, Av. Ejército Libertador 441, Santiago, Chile

${ }^{38}$ Koninklijke Sterrenwacht van België, Ringlaan 3, B-1180 Brussel, Belgium

${ }^{39}$ LESIA, UMR 8109, Observatoire de Paris et Université Pierre et Marie Curie Sorbonne Universités, place J. Janssen, 92195 Meudon, France

${ }^{40}$ Departamento de Física, Universidad Técnica Federico Santa María, Avda. España 1680, Valparaíso, Chile

${ }^{41}$ Astrophysics Group, Keele University, Staffordshire ST5 5BG, UK

${ }^{42}$ Institute of Astronomy and NAO, Bulgarian Academy of Sciences, blvd. Tsarigradsko chaussee 72, Sofia 1784, Bulgaria

${ }^{43}$ Department of Physics and Kavli Institute for Astrophysics and Space Research, Massachusetts Institute of Technology, Cambridge, MA 02139, USA

This paper has been typeset from a $\mathrm{T}_{\mathrm{E}} \mathrm{X} / \mathrm{LT} \mathrm{E} \mathrm{X}$ file prepared by the author. 